COR:TE

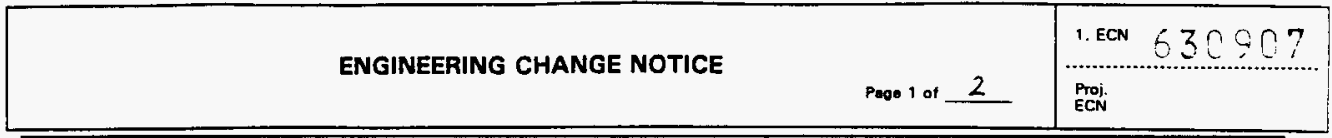

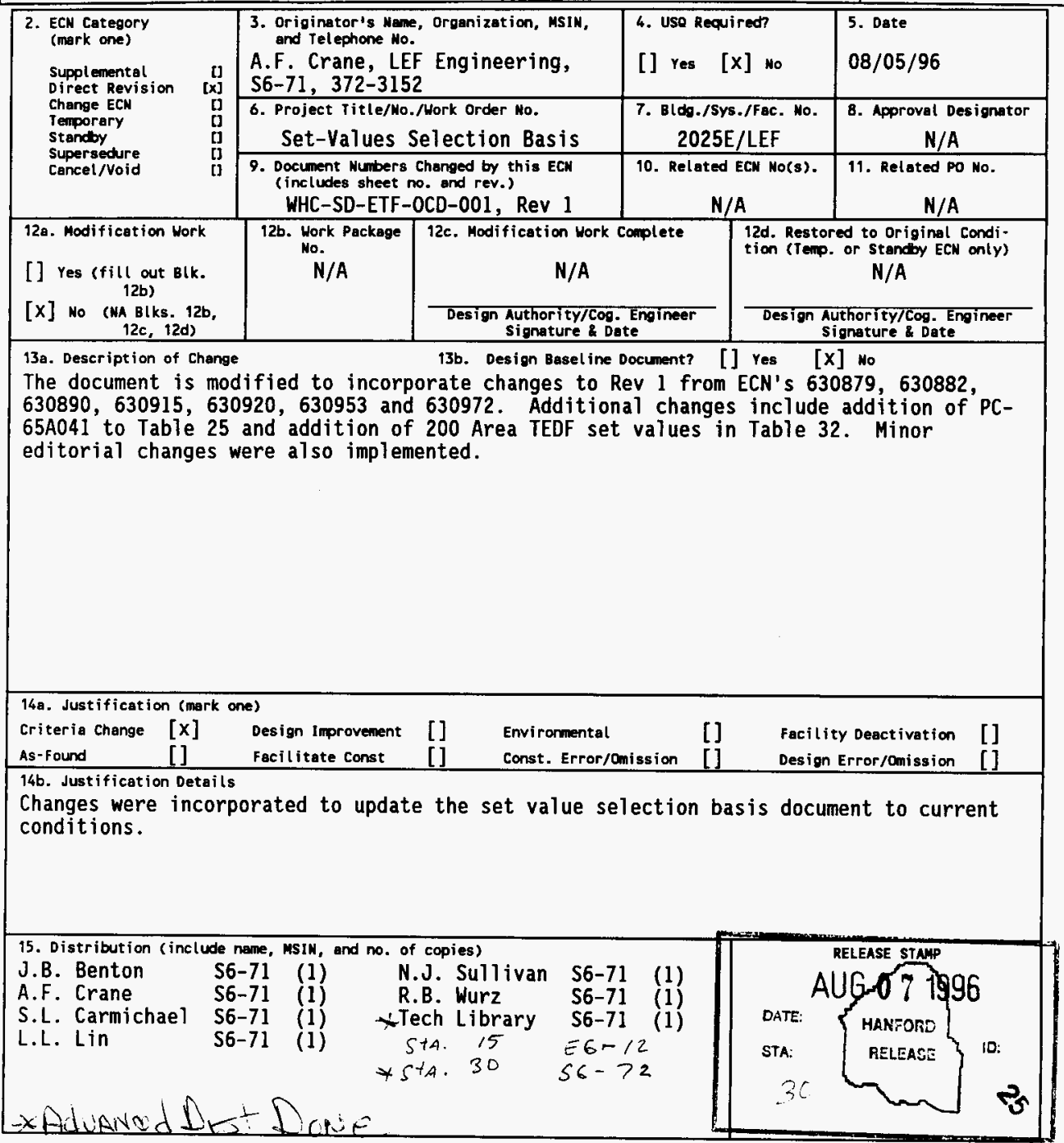

A-7900-013-2 (05/96) GEF095 


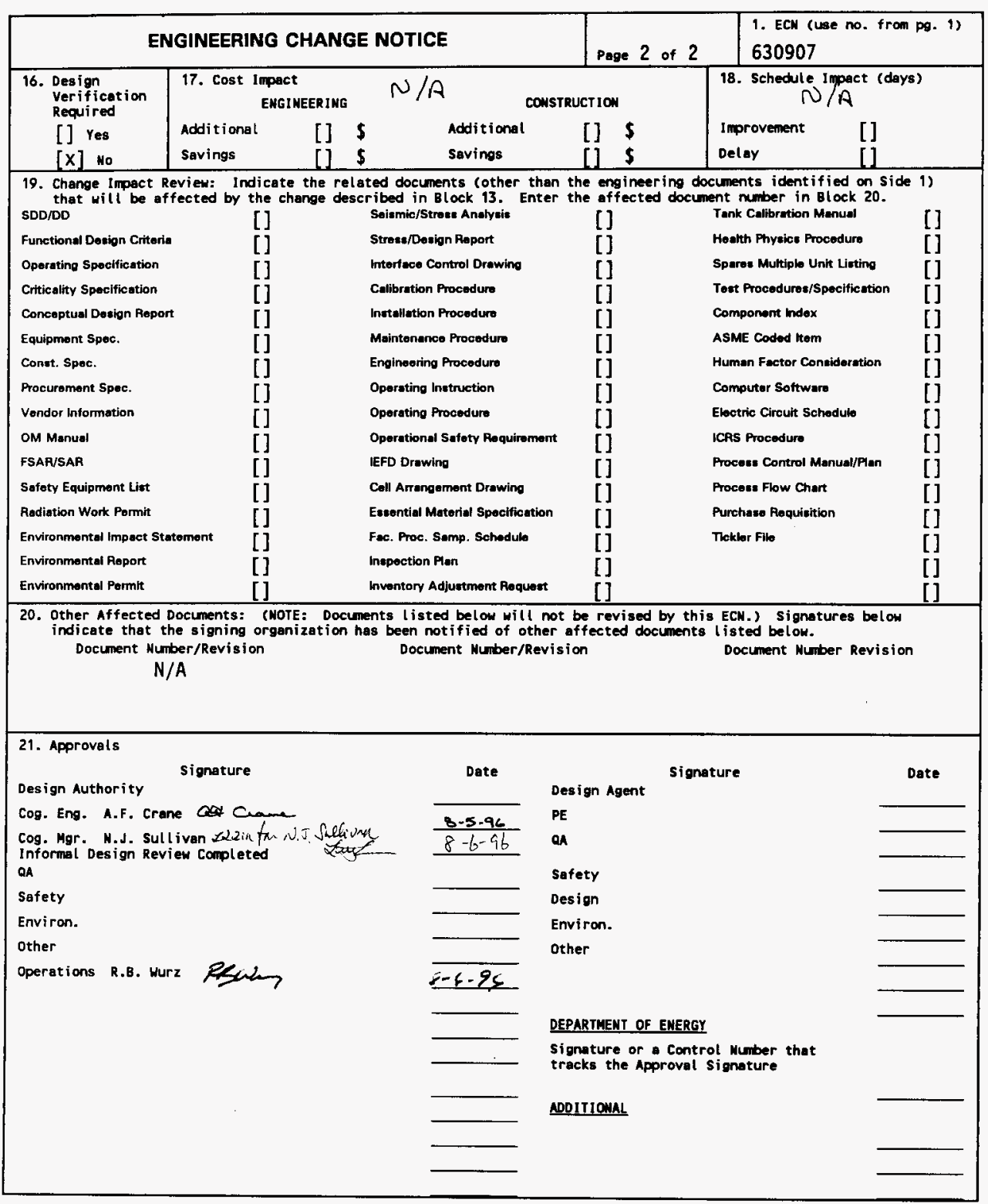




\section{Area Effluent Treatment Facility Set-Values Selection Basis}

\section{A.F. Crane}

Westinghouse Hanford Company, Richland, WA 99352

U.S. Department of Energy Contract DE-AC06-87RL10930

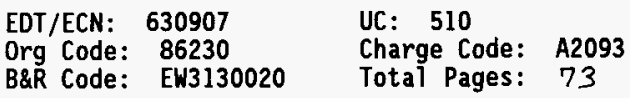

Key Words: 200 Area ETF, 200 Area LEF, 200 Area TEDF, Set-Values, Setpoint Values

Abstract: This document identifies the set-values and selection basis to support operation of the 200 Area ETF and 200 Area TEDF.

TRADEMARK DISCLAIMER. Reference herein to ony specific comnercial product, process, or service by trade neme, tradenark, manufacturer, or otherwise, does not necessarily constitute or imply its endorsement, recomendetion, or favoring by the United States Governaent or any agency thereof or its contractors or subcontractors.

Printed in the United States of America. To obtain copies of this document, contact: HHC/BCS Document Control Services, P.O. Box 1970, Mailstop H6-08, Richland WA 99352, Phone (509) 372-2420; Fax (509) 376-4989.
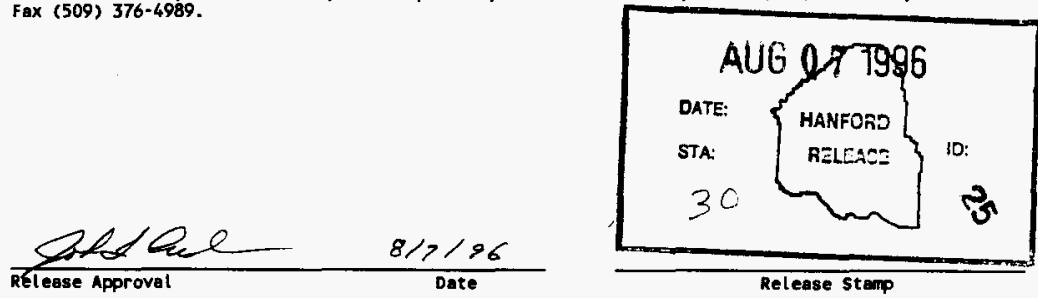

Approved for Public Release 
RECORD OF REVISION

(1) Document Nuiber

WHC-SD-ETF-OCD-

001 , Rev 2

(2) Title

200 Area Effluent Treatment Facility Set-Values Selection Basis

CHAMGE CONTROL RECORD

(3) Revision (4) Description of Change - Replace, Add, and Delete Pages

EDT 611986

I $\quad$ Revise per ECN \#622319 - Incorporate

applicable portions of ECN's 622247,

$627632,627640,622249$ and add comment to

pg 44, Table 16 for LIC-60I107.

2 Revise per ECN \# 630907 - Incorporate ECN's $630879,630882,630890,630915,630920$, 630953 and 630972 . Add PC-65A041 to Table 32. Minor editorial changes were also implemented. 
WHC-SD-ETF-OCD-001, Rev. 2

200 AREA EFFLUENT TREATMENT FACILITY

SET-VALUES SELECTION BASIS 
TABLE OF CONTENTS

$1.0 \quad$ PURPOSE $\ldots \ldots \ldots \ldots \ldots \ldots \ldots \ldots \ldots \ldots \ldots \ldots \ldots \ldots \ldots \ldots \ldots \ldots$

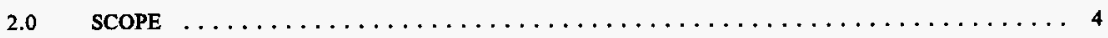

$3.0 \quad$ METHOD $\ldots \ldots \ldots \ldots \ldots \ldots \ldots \ldots \ldots \ldots \ldots \ldots \ldots \ldots \ldots \ldots \ldots \ldots \ldots$

TABLE $1 \quad 60 A$ INFLUENT RECEIVING \& STAGING (Surge Tank System) $\ldots \ldots \ldots \ldots \ldots \ldots$

TABLE $260 B$ FILTRATION (Rough Filter System) $\ldots \ldots \ldots \ldots \ldots \ldots \ldots \ldots \ldots \ldots \ldots$

TABLE $3 \quad 60 B$ FILTRATION (Fine Filter System) $\ldots \ldots \ldots \ldots \ldots \ldots \ldots \ldots \ldots \ldots \ldots$

TABLE $4 \quad 60 \mathrm{C}$ pH ADJUSTMENT (pH Adjustment Tank System) $\ldots \ldots \ldots \ldots \ldots \ldots \ldots \ldots$

TABLE $5 \quad 60 \mathrm{C} \mathrm{pH}$ ADJUSTMENT (Effluent $\mathrm{pH}$ Adjustment Tank System) $\ldots \ldots \ldots \ldots \ldots \ldots$

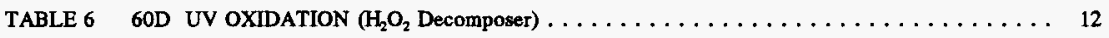

TABLE 6.1 60D UV OXIDATION (UV Oxidizet-1) $\ldots \ldots \ldots \ldots \ldots \ldots \ldots \ldots \ldots \ldots$

TABLE 6.2 60D UV OXIDATION (UV Oxidizer 2$) \ldots \ldots \ldots \ldots \ldots \ldots \ldots \ldots \ldots \ldots \ldots \ldots$

TABLE $6.360 \mathrm{D}$ UV OXIDATION $\left(\mathrm{H}_{2} \mathrm{O}_{2}\right.$ Feed System) $\ldots \ldots \ldots \ldots \ldots \ldots \ldots \ldots \ldots \ldots$

TABLE 7 60D UV OXIDATION SYSTEM $\left(\mathrm{H}_{2} \mathrm{O}_{2}\right.$ Decomposer System - by PSI $\ldots \ldots \ldots \ldots \ldots \ldots$

TABLE $8 \quad 60 E$ DEGASIFICATION SYSTEM $\ldots \ldots \ldots \ldots \ldots \ldots \ldots \ldots \ldots \ldots \ldots \ldots \ldots$

TABLE 9 60F REVERSE OSMOSIS (1st RO Feed System) $\ldots \ldots \ldots \ldots \ldots \ldots \ldots \ldots \ldots$

TABLE $10 \quad$ 60F REVERSE OSMOSIS $(1$ st RO Stage) $\ldots \ldots \ldots \ldots \ldots \ldots \ldots \ldots \ldots \ldots$

TABLE 11 60F REVERSE OSMOSIS (2nd RO Feed System) $\ldots \ldots \ldots \ldots \ldots \ldots \ldots \ldots$

TABLE 12 60F REVERSE OSMOSIS (2nd RO Stage) $\ldots \ldots \ldots \ldots \ldots \ldots \ldots \ldots \ldots \ldots \ldots$

TABLE 13 60F REVERSE OSMOSIS (RO/CIP System, Scale Inhibitor Dosing System) $\ldots \ldots \ldots \ldots$

TABLE $1460 G$ ION EXCHANGE SYSTEM (Polisher System) $\ldots \ldots \ldots \ldots \ldots \ldots \ldots$

TABLE $1560 \mathrm{H}$ EFFLUENT VERIFICATION (Verification System) $\ldots \ldots \ldots \ldots \ldots \ldots$

TABLE 16 60I WASTE EVAPORATION (Secondary Waste Receiving System) $\ldots \ldots \ldots \ldots \ldots$

TABLE 17 60I WASTE EVAPORATION (Evaporator System) $\ldots \ldots \ldots \ldots \ldots \ldots \ldots$

TABLE 18 60J WASTE DRYING (Concentrate Receiving System) $\ldots \ldots \ldots \ldots \ldots \ldots \ldots$

TABLE 19 60J WASTE DRYING (Dryer System) $\ldots \ldots \ldots \ldots \ldots \ldots \ldots \ldots \ldots \ldots \ldots$

TABLE $2080 \mathrm{C}$ DRUM HANDLING SYSTEM $\ldots \ldots \ldots \ldots \ldots \ldots \ldots \ldots \ldots \ldots$

TABLE 21 1B COMPRESSED AIR SYSTEM (Service Air) $\ldots \ldots \ldots \ldots \ldots \ldots \ldots \ldots$ 
TABLE 22 1D COMPRESSED AIR SYSTEM (Instrument Air) $\ldots \ldots \ldots \ldots \ldots \ldots \ldots$

TABLE $2320 B$ SUMP \& BUILDING DRAINS (Sump Tank System) $\ldots \ldots \ldots \ldots \ldots \ldots \ldots \ldots$

TABLE $24 \quad 45 D$ PROCESS OFFGAS (Vessel Ventilation System) $\ldots \ldots \ldots \ldots \ldots \ldots \ldots \ldots$

TABLE $25 \quad 65$ A STEAM SYSTEM $\ldots \ldots \ldots \ldots \ldots \ldots \ldots \ldots \ldots \ldots \ldots \ldots \ldots$

TABLE $2665 \mathrm{C}$ CHEMICAL MAKEUP AND STORAGE (Chemical Reagent Feed System) $\ldots \ldots \ldots \ldots$

TABLE 27 95B CLOSED LOOP COOLING (Seal Water System) $\ldots \ldots \ldots \ldots \ldots \ldots \ldots$

TABLE $2895 \mathrm{C}$ COOLING WATER (Cooling Water System) $\ldots \ldots \ldots \ldots \ldots \ldots \ldots \ldots \ldots$

TABLE 29 95D DEMIKERALIZED WATER SYSTEM $\ldots \ldots \ldots \ldots \ldots \ldots \ldots \ldots \ldots \ldots \ldots$

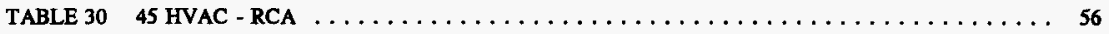

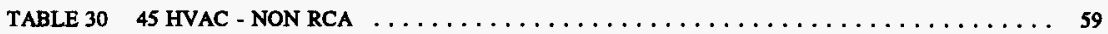

TABLE $31 \quad$ 45B HVAC CHILLED WATER (Chiller System) $\ldots \ldots \ldots \ldots \ldots \ldots \ldots \ldots \ldots$

TABLE 3268 - 200 AREA TREATED EFFLUENT DISPOSAL FACILITY $\ldots \ldots \ldots \ldots \ldots$

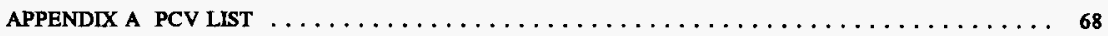

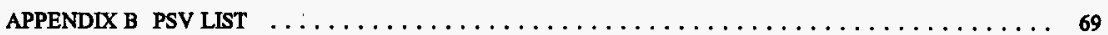

APPENDIX C RO DATA SHEET $\ldots \ldots \ldots \ldots \ldots \ldots \ldots \ldots \ldots \ldots \ldots \ldots \ldots \ldots \ldots \ldots \ldots$ 


\subsection{PURPOSE}

The purpose of this document is to provide the operational and alarm setpoints for the 200 Area Effluent Treatment Facility (ETF) and 200 Area Treated Effluent Disposal Facility (200 Area TEDF) Systems and Components. The setpoint values and bases are identified.

\section{$2.0 \quad$ SCOPE}

This Set-Values Selection Basis is specific to the ETF and 200 Area TEDF. This document provides a reference for the preparation of engineering, operating, maintenance documentation necessary for safe operations.

\subsection{METHOD}

Tables 1 through 32 provide the Set-Values, Functions, and the bases for the Set-Values for Systems and Components in the ETF and 200 Area TEDF. In addition Appendices A, B, and C provide lists of Pressure Control Valves (PCV), Pressure Safety Valves (PSV) and Restricting Orifices (RO) in the ETF. 
TABLE 1 60A INFLUENT RECEIVING \& STAGING (Surge Tank System)

\begin{tabular}{|c|c|c|c|c|}
\hline \multicolumn{2}{|c|}{$\begin{array}{l}\text { Tag No. (PID No. } \\
\text { H-2-89337) }\end{array}$} & \multirow{2}{*}{$\begin{array}{l}\text { Name } \\
\begin{array}{l}\text { PUREX Plant PDD, } \\
\text { ASD Flow }\end{array} \\
\end{array}$} & Set-Values and Functions & Remarks \\
\hline FI & $\begin{array}{r}-60 \mathrm{~A} 001 \\
(88974) \\
\end{array}$ & & $\begin{array}{l}\text { Operating Range: } 20-75 \mathrm{gpm} \\
\text { SH : } 20 \mathrm{gpm} \text { Start Feed forward control }\end{array}$ & $\begin{array}{l}\text { Flowrate of mixed influent } 40-150 \mathrm{gpm} \\
\text { PDD or ASD is equally mixed with LERF or PC } \\
40 / 2-150 / 2=20-75\end{array}$ \\
\hline FI & $\begin{array}{r}-60 A 002 \\
(88974) \\
\end{array}$ & $\begin{array}{l}\text { Retention } \\
\text { Basins(LERF) Flow }\end{array}$ & $\begin{array}{l}\text { Operating Range: } 20-150 \mathrm{gpm} \\
\text { SH : } 20 \text { gpm Start Feed forward control }\end{array}$ & $\begin{array}{cl}40 / 2=20 & \begin{array}{l}\text { (Equally mixed with PDD or } \\
\text { ASD) } \\
\text { (Maximum flowrate of mixed } \\
\text { influent) }\end{array} \\
\end{array}$ \\
\hline FI & $\begin{array}{r}-60 A 003 \\
(88974) \\
\end{array}$ & $\begin{array}{l}\text { 242-A Evaporator } \\
\text { (PC) Flow }\end{array}$ & $\begin{array}{l}\text { Operating Range: } 20-75 \mathrm{gpm} \\
\text { SH : } 20 \mathrm{gpm} \text { Start Feed forward control }\end{array}$ & $\begin{array}{cl}40 / 2=20 & \text { (Equally mixed with PDD or } \\
75 & \begin{array}{l}\text { ASD) } \\
\text { (Maximum capacity of others- } \\
\text { installed transfer system) }\end{array} \\
\end{array}$ \\
\hline $\mathbf{R I}$ & $-60 A 004$ & $\begin{array}{l}\text { Receiving Line } \\
\text { Radiation }\end{array}$ & $\begin{array}{l}\text { Operating Range: } 0-8.6 \mathrm{E}-4 \mathrm{Ci} / \mathrm{m} 3 \\
\mathrm{AH}: 1.3 \mathrm{E}-3 \text { Alarm }\end{array}$ & $\begin{array}{l}\text { PDD: } \quad 5.2 \mathrm{E}-4 \mathrm{Ci} / \mathrm{m}^{3} \\
\text { ASD: } \quad 8.6 \mathrm{E}-4 \mathrm{Ci} / \mathrm{m}^{3} \\
\text { PC/LERF: } 8.7 \mathrm{E}-5 \mathrm{Ci} / \mathrm{m}^{3} \\
\text { 4.5*(Maximum) } \\
\text { Provide monitor trouble and failure indication }\end{array}$ \\
\hline $\begin{array}{l}\text { AI } \\
\text { (COND) }\end{array}$ & $\begin{array}{r}-60 \mathrm{~A} 005 \\
(88974) \\
\end{array}$ & $\begin{array}{l}\text { Receiving Line } \\
\text { Conductivity }\end{array}$ & Operating Range: $0-1060 \mathrm{E}-6 \mathrm{~S} / \mathrm{cm}$ & $\begin{array}{l}\text { PDD: } \quad 1060 \mathrm{E}-6 \mathrm{~S} / \mathrm{cm} \\
\text { ASD: } \quad 300 \\
\text { PC/LERF: }(590)\end{array}$ \\
\hline $\begin{array}{l}\text { AI } \\
(\mathrm{pH})\end{array}$ & $-60 \mathrm{~A} 006$ & Receiving Line $\mathrm{pH}$ & $\begin{array}{l}\text { Operating Range: } 0-14 \\
\text { SHH: } 6.5 \text { Start Titration Analyzer } \\
\text { SH : 6.0 Stop Titration Analyzer } \\
\text { SL }: 6.0 \text { Stop NaOH Control } \\
\text { SLL: } 5.0 \text { Start NaOH Control } \\
\end{array}$ & $\begin{array}{l}\text { PDD: 2.28, ASD: } 9.66, \\
\text { PC/LERF: } 10.1-11.3\end{array}$ \\
\hline $\begin{array}{l}\text { AI } \\
\text { (TOC) }\end{array}$ & $\begin{array}{r}-60 A 007 \\
(88974) \\
\end{array}$ & Receiving Line TOC & Operating Range: $0-218 \mathrm{ppm}$ & $\begin{array}{l}\text { PDD: } \quad 130 \mathrm{ppm} \\
\text { ASD: } \quad 4.8 \\
\text { PC/LERF: } 218 \quad(90 \% \mathrm{CI})\end{array}$ \\
\hline & $\begin{array}{r}-60 A 011 \\
(89337)\end{array}$ & $\begin{array}{l}\text { Surge Tank } \\
\text { Temperature }\end{array}$ & $\begin{array}{ll}\mathrm{AH}: 190^{\circ} \mathrm{F} & \text { Alarm } \\
\text { SH }: 50^{\circ} \mathrm{F} & \text { Tum off freeze-protection heaters } \\
\text { SL }: 40^{\circ} \mathrm{F} & \text { Turn on freeze-protection heaters } \\
\mathrm{AL}: 35^{\circ} \mathrm{F} & \text { Alarm }\end{array}$ & $\begin{array}{l}\text { Lower than max. operating temperature } 200^{\circ} \mathrm{F} \\
\mathrm{SL}+10^{\circ} \mathrm{F} \\
\text { Maximum density point of water }\end{array}$ \\
\hline
\end{tabular}




\begin{tabular}{|c|c|c|c|}
\hline $\begin{array}{l}\text { Tag No. (PID No. } \\
\text { H-2-89337) }\end{array}$ & Name & Set-Values and Functions & Remarks \\
\hline $\begin{array}{r}-60 \mathrm{A012} \\
\\
\\
(89337) \\
\end{array}$ & Surge Tank Level & $\begin{array}{ll}\text { AH }: 95 \% & \begin{array}{l}\text { Alarm } \\
\text { Change Evaporator and Dryer from }\end{array} \\
\text { "OPERATION" to "READY" } \\
\text { SHH : } 93 \% & \begin{array}{l}\text { Close AOV-60A054,055,056 } \\
\text { Stop others-installed transfer pumps }\end{array} \\
\text { SL :(70 \%) } & \text { Starting condition of Polisher regeneration } \\
\text { SLL }: 7 \% & \text { Close AOV-60A057 } \\
\text { AL : } 5 \% & \text { Alarm } \\
& \text { Stop pumps 2025E-60A-P-1A,B,C } \\
& \text { Turn off freeze-protection heaters }\end{array}$ & See Equipment Process Calculation \\
\hline $\begin{array}{r}-60 A 013 \\
(89337) \\
\end{array}$ & Surge Tank Level & AH : $97 \% \quad$ Alarm & $\begin{array}{l}\text { Back-up for LI-60A012 } \\
\text { H(LI-60A012) }+2 \% \\
\end{array}$ \\
\hline $\begin{array}{r}-60 A 014 \\
(89337) \\
\end{array}$ & $\begin{array}{l}\text { Surge Tank Pump } \\
\text { Discharge Pressure }\end{array}$ & Operating : $95 \sim 105$ psig & System Design \\
\hline $\begin{array}{r}-60 \mathrm{A021} \\
(88974) \\
\end{array}$ & $\begin{array}{l}\text { On-Line Titration } \\
\text { Analyzer }\end{array}$ & $\mathrm{SV}: \mathrm{pH}=6$ & $\begin{array}{l}\text { To prevent ammonia from being entrained to } \\
\text { Vessel Ventilation System } \\
\end{array}$ \\
\hline $\begin{array}{r}-60 \mathrm{~A} 022 \\
(89337)\end{array}$ & Surge Tank pH & 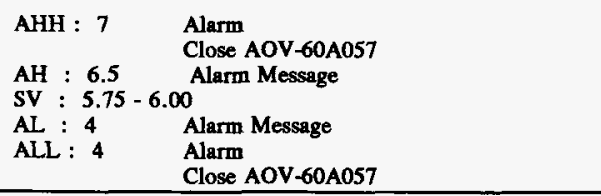 & $\begin{array}{l}H+0.5 \\
\text { SV(On-Line Titration Analyzer) }+0.5 \\
\text { SV(On-Line Titration Analyzer) }-1 \\
\text { L- } 1\end{array}$ \\
\hline $\begin{array}{rr}\text { AI } \quad-60 \mathrm{A023}(\mathrm{pH}) \\
(89337) \\
\end{array}$ & Surge Tank pH & Same as Al-60A022 & Same as AI-60A022 \\
\hline $\begin{array}{r}-60 \mathrm{~A} 032 \\
(89337) \\
\end{array}$ & $\begin{array}{l}\text { Surge Tank Pump } \\
\text { (A) Current }\end{array}$ & FLA : $42.1 \mathrm{~A}$ & $\begin{array}{l}\text { V-1358-002-001 } \\
\text { FLA: Full Load Amperage }\end{array}$ \\
\hline $\begin{array}{r}-60 \mathrm{~A} 034 \\
(89337) \\
\end{array}$ & $\begin{array}{l}\text { Surge Tank Pump } \\
\text { (B) Current }\end{array}$ & FLA : 42.1 A & $\begin{array}{l}\text { V-1358-002-001 } \\
\text { FLA: Full Load Amperage }\end{array}$ \\
\hline $\begin{array}{r}-60 A 036 \\
(89337)\end{array}$ & $\begin{array}{l}\text { Surge Tank Pump } \\
\text { (C) Current }\end{array}$ & FLA : $42.1 \mathrm{~A}$ & $\begin{array}{l}\text { V-1358-002-001 } \\
\text { FLA: Full Load Amperage }\end{array}$ \\
\hline
\end{tabular}


TABLE 2 60B FILTRATION (Rough Filter System)

\begin{tabular}{|c|c|c|c|c|}
\hline \multicolumn{2}{|c|}{$\begin{array}{l}\text { Tag No. (PID No. } \\
\text { H-2-89337) }\end{array}$} & \multirow{2}{*}{$\begin{array}{l}\text { Name } \\
\text { UV Oxidation } \\
\text { Inlet Temperature }\end{array}$} & Set-Values and Functions & \multirow{2}{*}{$\begin{array}{l}\text { Remarks } \\
\text { Prevent Damage from Overheating. } \\
\text { Prevent Damage from Freezing. }\end{array}$} \\
\hline TI & $\begin{array}{r}-60 \mathrm{~B} 004 \\
(88975) \\
\end{array}$ & & $\begin{array}{lll}\mathrm{AH} & : 120^{\circ} \mathrm{F} & \text { Alarm } \\
\mathrm{AL}: 40^{\circ} \mathrm{F} & \text { Alarm }\end{array}$ & \\
\hline Tw & $\begin{array}{l}-60 \mathrm{~B} 007 \\
\mathbf{( 8 8 9 7 5 )}\end{array}$ & $\begin{array}{l}\text { UV Oxidation Inlet } \\
\text { Cooler Waste Water } \\
\text { Inlet Temperature }\end{array}$ & Operating : $<140^{\circ} \mathrm{F}$ & System Design Process Value. \\
\hline TW & $\begin{array}{l}-60 \mathrm{~B} 008 \\
(88975) \\
\end{array}$ & $\begin{array}{l}\text { UV Oxidation Inlet } \\
\text { Cooler Waste Water } \\
\text { Outlet Temperature }\end{array}$ & Operating : $<100^{\circ} \mathrm{F}$ & System Design Process Value. \\
\hline Tw & $\begin{array}{r}-60 \mathrm{~B} 009 \\
(88975)\end{array}$ & $\begin{array}{l}\text { UV Oxidation Inlet } \\
\text { Cooler Cooling } \\
\text { Water Outlet } \\
\text { Temperature }\end{array}$ & Operating : $112^{\circ} \mathrm{F}$ & System Design Process Value. \\
\hline Tw & $\begin{array}{r}-60 \mathrm{~B} 010 \\
(88975) \\
\end{array}$ & $\begin{array}{l}\text { UV Oxidation Inlet } \\
\text { Cooler Cooling } \\
\text { Water Inlet } \\
\text { Temperature } \\
\end{array}$ & Operating : $76^{\circ} \mathrm{F}$ & System Design Process Value. \\
\hline $\begin{array}{l}\text { PI } \\
\text { (Local) }\end{array}$ & $\begin{array}{r}-60 \mathrm{~B} 023 \\
(88975) \\
\end{array}$ & $\begin{array}{l}\text { Service Air } \\
\text { Regulator } \\
\text { Outlet Pressure }\end{array}$ & Operating : 90 psig & $\begin{array}{l}\text { System Design Process Value. } \\
\text { V-135A-001-A-694 }\end{array}$ \\
\hline PI & $\begin{array}{r}-60 \mathrm{~B} 101 \\
(88975) \\
\end{array}$ & $\begin{array}{l}\text { Rough Filter } \\
\text { Inlet Pressure }\end{array}$ & Operating Range : $50-100$ psig & $\begin{array}{l}\text { System Design. } \\
\text { V-135A-001-A-694 }\end{array}$ \\
\hline PI & $\begin{array}{r}-60 \mathrm{~B} 102 \\
(88975) \\
\end{array}$ & $\begin{array}{l}\text { Rough Filter } \\
\text { Outlet Pressure } \\
\end{array}$ & Operating Range $: 20-100$ psig & $\begin{array}{l}\text { System Design. } \\
\text { V-135A-001-A-694 } \\
\end{array}$ \\
\hline PDI & $\begin{array}{r}-60 \mathrm{~B} 103 \\
(88975) \\
\end{array}$ & $\begin{array}{l}\text { Rough Filter } \\
\text { Differential Pressure }\end{array}$ & $\begin{array}{ll}\text { AH: } & 80 \text { psi Alarm } \\
\text { SH: } & 5-50 \text { psi as determined by cognizant engineer } \\
& \text { Tum to Backwash operation } \\
\end{array}$ & $\begin{array}{l}\text { PI60B101 - PI60B102: Calculation by MCS } \\
\text { Clean Pressure Drop: } 2.5 \text { psi } \\
\text { V-135A-001-A-694 }\end{array}$ \\
\hline PI & $\begin{array}{r}-60 \mathrm{~B} 105 \\
(88975) \\
\end{array}$ & $\begin{array}{l}\text { Rough Filter } \\
\text { Service Air Pressure } \\
\end{array}$ & $\begin{array}{l}\text { Operating : } 90 \text { psig } \\
\text { AL: } \quad 75 \text { psig Alarm }\end{array}$ & $\begin{array}{l}\text { Filter Backwash Pressure }=90 \text { psig } \\
\text { V-135A-001-A-694 }\end{array}$ \\
\hline LS & $\begin{array}{r}-60 \mathrm{~B} 111 \\
(88975) \\
\end{array}$ & $\begin{array}{l}\text { Rough Filter } \\
\text { Lower Level }\end{array}$ & $\begin{array}{ll}\text { SH: } & \text { ON - Close lower vent valve, AOV-60B007 } \\
\text { Open upper vent valve, AOV-60B009 }\end{array}$ & $\begin{array}{l}\text { Detect the fluid fill beneath the tubesheet of the } \\
\text { vessel. V-135A-001-A-694 }\end{array}$ \\
\hline LS & $\begin{array}{r}-60 \mathrm{~B} 112 \\
(88975)\end{array}$ & $\begin{array}{l}\text { Rough Filter } \\
\text { Upper Level }\end{array}$ & $\begin{array}{ll}\text { SH: } & \text { ON - Open outlet valve, AOV-60B015 } \\
& \text { Close upper vent valve, AOV-60B009 }\end{array}$ & $\begin{array}{l}\text { Detect the fluid fill the upper vessel. } \\
\text { V-135A-001-A-694 }\end{array}$ \\
\hline
\end{tabular}


TABLE 3 60B FILTRATION (Fine Filter System)

\begin{tabular}{|c|c|c|c|c|}
\hline \multicolumn{2}{|c|}{$\begin{array}{l}\text { Tag No. (PID No. } \\
\text { H-2-89337) }\end{array}$} & \multirow{2}{*}{\begin{tabular}{|l|} 
Name \\
$\begin{array}{l}\text { 1st RO Feed Tank } \\
\text { Inlet Turbidity }\end{array}$ \\
\end{tabular}} & Set-Values and Functions & \multirow{2}{*}{$\begin{array}{l}\text { Remarks } \\
\text { NTU: Nephelometric Turbidity Units } \\
\text { System Design Process Value. }\end{array}$} \\
\hline $\begin{array}{l}\text { AI } \\
\text { (TURB) }\end{array}$ & $\begin{array}{r}-60 \mathrm{~B} 012 \\
(89334) \\
\end{array}$ & & Operating Range: $<10 \mathrm{NTU}$ & \\
\hline $\begin{array}{l}\text { TW } \\
\quad(8933 \\
\end{array}$ & $-60 \mathrm{~B} 030$ & $\begin{array}{l}\text { Degasification } \\
\text { Column Inlet Cooler } \\
\text { waste Water Outlet } \\
\text { Temperature }\end{array}$ & Operating : $86 \mathrm{~F}$ & System Design Process Value. \\
\hline $\begin{array}{r}\text { TW } \\
\quad 8833 \\
\end{array}$ & $-60 \mathrm{~B} 031$ & $\begin{array}{l}\text { Degasification } \\
\text { Column Inlet Cooler } \\
\text { Waste Water Inlet } \\
\text { Temperature } \\
\end{array}$ & Operating : $140 \mathrm{~F}$ & System Design Process Value \\
\hline $\begin{array}{l}\text { TW } \\
\quad(8933\end{array}$ & $-60 \mathrm{~B} 032$ & $\begin{array}{l}\text { Degasification } \\
\text { Column Inlet Cooler } \\
\text { Cooling Water Outlet } \\
\text { Temperature }\end{array}$ & Operating : $112 \mathrm{~F}$ & System Design Process Value \\
\hline Tw & $\begin{array}{r}-60 \mathrm{~B} 033 \\
(89334) \\
\end{array}$ & $\begin{array}{l}\text { Degasification } \\
\text { Column Inlet Cooler } \\
\text { Cooling Water Inlet } \\
\text { Temperature } \\
\end{array}$ & Operating : $76 \mathrm{~F}$ & System Design Process Value \\
\hline $\begin{array}{l}\text { PI } \\
\text { (Local) }\end{array}$ & $\begin{array}{r}-60 \mathrm{~B} 053 \\
(88978)\end{array}$ & $\begin{array}{l}\text { Service Air } \\
\text { Regulator } \\
\text { Outlet Pressure }\end{array}$ & Operating : 90 psig & $\begin{array}{l}\text { System Design Process Value. } \\
\text { V-135A-001-A-694 }\end{array}$ \\
\hline PI & $\begin{array}{r}-60 \mathrm{~B} 201 \\
(88978)\end{array}$ & $\begin{array}{l}\text { Fine Filter } \\
\text { Inlet Pressure }\end{array}$ & Operating : $0 \sim 60$ psig & $\begin{array}{l}\text { System Design. } \\
\text { V-135A-001-A-694 }\end{array}$ \\
\hline PI & $\begin{array}{r}-60 \mathrm{~B} 202 \\
(88978)\end{array}$ & $\begin{array}{l}\text { Fine Filter } \\
\text { Outlet Pressure }\end{array}$ & Operating : $0 \sim 60$ psig & $\begin{array}{l}\text { System Design. } \\
\text { V-135A-001-A-694 }\end{array}$ \\
\hline PDI & $\begin{array}{r}-60 \mathrm{~B} 203 \\
(88978)\end{array}$ & $\begin{array}{l}\text { Fine Filter } \\
\text { Differential Pressure }\end{array}$ & $\begin{array}{ll}\text { AH: } & 80 \text { psi Alarm } \\
\text { SH: } & 5-50 \text { psi as determined by cognizant engineer } \\
& \text { Turn to Backwash operation }\end{array}$ & $\begin{array}{l}\text { PI60B201 - PI60B202: Calculation By MCS } \\
\text { Clean Pressure Drop: } 2.5 \text { psi }\end{array}$ \\
\hline PI & $\begin{array}{r}-608205 \\
(88978) \\
\end{array}$ & $\begin{array}{l}\text { Fine Filter } \\
\text { Service Air Pressure }\end{array}$ & $\begin{array}{l}\text { Operating : } 90 \text { psig } \\
\text { AL: } \quad 75 \text { psig Alarm }\end{array}$ & $\begin{array}{l}\text { Filter Backwash Pressure }=90 \text { psig } \\
\text { V-135A-001-A-694 }\end{array}$ \\
\hline LS & $\begin{array}{r}-60 \mathrm{~B} 211 \\
(88978)\end{array}$ & $\begin{array}{l}\text { Fine Filter } \\
\text { Lower Level }\end{array}$ & $\begin{array}{l}\text { SH: } \\
\text { ON - Close lower vent valve AOV-60B037 } \\
\text { Open upper vent valve AOV-60B039 }\end{array}$ & $\begin{array}{l}\text { Detect the fluid fill beneath the tubesheet of the } \\
\text { vessel. } \\
\text { V-135A-001-A-694 }\end{array}$ \\
\hline
\end{tabular}


WHC-SD-ETF-OCD-001, Rev. 2

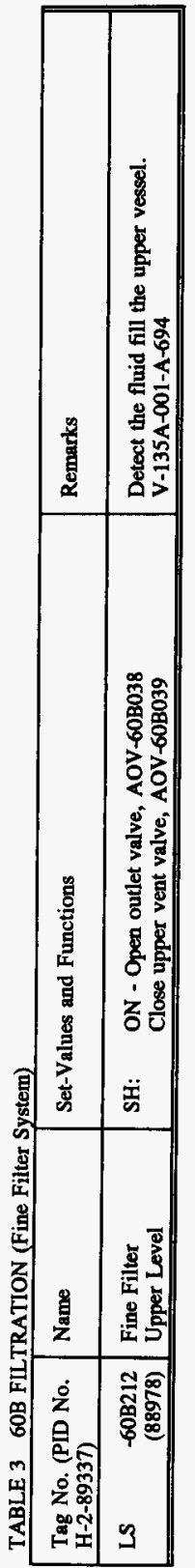


TABLE $4 \quad 60 \mathrm{C}$ pH ADJUSTMENT (pH Adjustment Tank System)

\begin{tabular}{|c|c|c|c|c|c|}
\hline \multicolumn{2}{|c|}{$\begin{array}{l}\text { Tag No. (PID No. } \\
\text { H-2-89337) }\end{array}$} & \multirow{2}{*}{$\begin{array}{l}\text { Name } \\
\begin{array}{l}\text { pH Adjustment Tank } \\
\text { Inlet pH }\end{array}\end{array}$} & \multicolumn{2}{|c|}{ Set-Values and Functions } & Remarks \\
\hline $\begin{array}{l}\mathrm{AI} \\
(\mathrm{pH})\end{array}$ & $\begin{array}{r}-60 C 101 \\
(88977) \\
\end{array}$ & & \multicolumn{2}{|c|}{ Operating : $4 \sim 6$} & System Design Process Value \\
\hline $\begin{array}{l}\text { AI } \\
(\mathrm{pH})\end{array}$ & $\begin{array}{r}-60 C 102 \\
(88977) \\
\end{array}$ & $\begin{array}{l}\text { pH Adjustment Tank } \\
\text { Inlet } \mathrm{pH}\end{array}$ & \multicolumn{2}{|c|}{ Same as $\mathrm{AI}-60 \mathrm{C} 101$} & System Design Process Value \\
\hline $\begin{array}{l}\text { AI } \\
\text { (pH) }\end{array}$ & $-60 \mathrm{C} 103$ & $\begin{array}{l}\text { pH Adjustment Tank } \\
\text { Outlet pH }\end{array}$ & $\begin{array}{l}\text { AHH : } 6 \\
\text { AH : } 5 \\
\text { AL : } 3 \\
\text { ALL : } 2\end{array}$ & $\begin{array}{l}\text { Alarm } \\
\text { Change UV System loop "OPERATION" to } \\
\text { "READY" } \\
\text { Alarm Message } \\
\text { Alarm Message } \\
\text { Alarm } \\
\text { Change UV System loop "OPERATION" to } \\
\text { "READY" }\end{array}$ & $\begin{array}{l}\text { AH }+1 \\
\text { SV }+1 \\
\text { To promote } \mathrm{CO}_{2} \text { removal in Degasification System } \\
\text { To prevent ammonia from being entrained by } \\
\text { permeate in RO System } \\
\text { SV }-1 \\
\text { L }-1\end{array}$ \\
\hline $\begin{array}{l}\text { Alc } \\
(\mathrm{pH})\end{array}$ & $\begin{array}{r}-600103 \\
(88977) \\
\end{array}$ & $\begin{array}{l}\text { pH Adjustment } \\
\text { Tank Outlet pH }\end{array}$ & \multicolumn{2}{|l|}{ SV : 4} & System Design Process Value \\
\hline $\begin{array}{l}\mathrm{AI} \\
(\mathrm{pH})\end{array}$ & $\begin{array}{r}-60 C 104 \\
(88977) \\
\end{array}$ & $\begin{array}{l}\text { pH Adjustment Tank } \\
\text { Outlet pH }\end{array}$ & \multicolumn{2}{|c|}{ Same as $\mathrm{AI} \cdot 60 \mathrm{C} 103$} & Back-Up of AIC - $60 \mathrm{C} 103$ \\
\hline LIC & $(88977)$ & $\begin{array}{l}\text { pH Adjustment Tank } \\
\text { Level }\end{array}$ & $\begin{array}{l}\text { AH }: 95 \% \\
\text { SV }: 50 \% \\
\text { AL : } 10 \%\end{array}$ & $\begin{array}{l}\text { Alarm } \\
\text { Change UV loop from "OPERATION" to } \\
\text { "READY" } \\
\text { Alarm } \\
\text { Stop pH Adjustment Tank Pumps 2025E- } \\
\text { 60C-P-1A,B }\end{array}$ & $\begin{array}{l}\text { See Equipment Process Calculation } \\
\text { Control of Tank Level during Processing }\end{array}$ \\
\hline TI & $\begin{array}{r}-60 C 112 \\
(88977) \\
\end{array}$ & $\begin{array}{l}\text { pH Adjustment Tank } \\
\text { Temperature }\end{array}$ & \multicolumn{2}{|c|}{ Operating Range: $90-140 \mathrm{~F}$} & $\begin{array}{l}\text { SV(TIC-60B004) }+40 \mathrm{~F} \\
\text { Temperature increase of UV Oxidizer: } 40 \mathrm{~F}\end{array}$ \\
\hline PI & $\begin{array}{r}-60 \mathrm{C} 121 \\
(88977) \\
\end{array}$ & $\begin{array}{l}\text { pH Adjustment Tank } \\
\text { Pump } \\
\text { Discharge Pressure }\end{array}$ & \multicolumn{2}{|c|}{ Operating: $110 \sim 120$ psig } & $\begin{array}{l}\text { System Design Process Value. Pump Design. } \\
\text { V-135B-002-B-039 }\end{array}$ \\
\hline II & $\begin{array}{r}-60 C 132 \\
(88977) \\
\end{array}$ & $\begin{array}{l}\text { pH Adjustment Tank } \\
\text { Pump (A) Current } \\
\end{array}$ & \multicolumn{2}{|l|}{ FLA : $50 \mathrm{~A}$} & $\begin{array}{l}\text { V-1358-002-313 } \\
\text { FLA: Full Load Amperage }\end{array}$ \\
\hline II & $\begin{array}{r}-60 C 134 \\
(88977)\end{array}$ & $\begin{array}{l}\text { pH Adjustment Tank } \\
\text { Pump (B) Current }\end{array}$ & \multicolumn{2}{|l|}{ FLA : $50 \mathrm{~A}$} & $\begin{array}{l}\text { V-1358-002-313 } \\
\text { FLA: Full Load Amperage }\end{array}$ \\
\hline
\end{tabular}




\begin{tabular}{|c|c|c|c|c|}
\hline \multicolumn{2}{|c|}{$\begin{array}{l}\text { Tag No. (PID } \\
\text { No.H-2-89337) } \\
\end{array}$} & \multirow{2}{*}{$\begin{array}{l}\text { Name } \\
\text { Effluent pH } \\
\text { Adjustment Tank } \\
\text { Level }\end{array}$} & Set-Values and Functions & \multirow{2}{*}{$\begin{array}{l}\text { Remarks } \\
\text { See Equipment Process Calculation } \\
\text { Tank Level Control During Processing }\end{array}$} \\
\hline LIC & $\begin{array}{r}-60 C 211 \\
(88984) \\
\end{array}$ & & $\begin{array}{ll}\text { AH : }: 95 \% & \begin{array}{l}\text { Alarm - Change RO loop from } \\
\text { "OPERATION" to "READY" }\end{array} \\
\text { SV : } 50 \% & \\
\text { AL : } 10 \% & \begin{array}{l}\text { Alarm - Stop Effluent pH Adjustment Tank } \\
\text { Pumps 2025E-60C-P-2A,B }\end{array} \\
\end{array}$ & \\
\hline TI & $\begin{array}{r}-60 C 212 \\
(88984)\end{array}$ & $\begin{array}{l}\text { Effluent pH } \\
\text { Adjustment Tank } \\
\text { Temperature }\end{array}$ & Operation Range: $50-120 \mathrm{~F}$ & $\begin{array}{l}\text { Clawson Design Tank Temperature. } \\
\text { V.1354-001-001 }\end{array}$ \\
\hline FIC & $\begin{array}{l}-60 C 218 \\
(88984) \\
\end{array}$ & $\begin{array}{l}\text { Effluent pH } \\
\text { Adjustment Tank } \\
\text { Outlet Flow }\end{array}$ & $\begin{array}{l}\text { Operating : } 40-150 \mathrm{gpm} \\
\text { SV: LIC-60C211 }\end{array}$ & $\begin{array}{l}\text { Process Design Flow. } \\
\text { V-1354-001-001 }\end{array}$ \\
\hline PI & $\begin{array}{r}-60 C 221 \\
(88984)\end{array}$ & $\begin{array}{l}\text { Effluent pH } \\
\text { Adjustment Tank } \\
\text { Pump Discharge } \\
\text { Pressure }\end{array}$ & Operating: $80 \mathrm{psig}$ (at $150 \mathrm{gpm}$ ) & $\begin{array}{l}\text { Pump Design. } \\
\text { V-1358-002-001 }\end{array}$ \\
\hline $\begin{array}{l}\mathrm{AI} \\
(\mathrm{pH})\end{array}$ & $\begin{array}{r}-60 C 222 \\
(88984)\end{array}$ & $\begin{array}{l}\text { Effluent pH } \\
\text { Adjustment Tank } \\
\text { Outlet pH }\end{array}$ & $\begin{array}{l}\text { AHH : } 14.0 \quad \text { Alarm - Close FCV }-60 \mathrm{C} 218 \\
\text { AH : } 14.0 \quad \text { Alarm message - Inject } 1 \text { batch of } \mathrm{H}_{2} \mathrm{SO}_{4} \\
\text { AL }: 0.0 \quad \text { Alarm message - Inject } 1 \text { batch of } \mathrm{NaOH} \\
\text { ALL: } 0.0 \text { Alarm - Close FCV-60C218 }\end{array}$ & $\begin{array}{l}\text { Discharge range: } 6.5-8.5 \\
\text { Expected value : } 7 \\
\mathrm{H}_{2} \mathrm{SO}_{4}: 0.04 \text { wt } \%, \mathrm{NaOH}: 0.2 \mathrm{wt} \%\end{array}$ \\
\hline $\begin{array}{l}\mathrm{AI} \\
(\mathrm{pH})\end{array}$ & $\begin{array}{r}-60 C 223 \\
(88984)\end{array}$ & $\begin{array}{l}\text { Effluent pH } \\
\text { Adjustment Tank } \\
\text { Outlet pH }\end{array}$ & Same as $\mathrm{AI}-60 \mathrm{C} 222$ & Back-Up for AI- $60 \mathrm{C} 222$ \\
\hline $\begin{array}{l}\text { AI } \\
\text { (COND) }\end{array}$ & $\begin{array}{r}-60 C 227 \\
(88984)\end{array}$ & $\begin{array}{l}\text { Effluent pH } \\
\text { Adjustment Tank } \\
\text { Outlet Conductivity }\end{array}$ & $\begin{array}{l}\text { Operating Range : } 0-5 \mathrm{E}-6 \mathrm{~S} / \mathrm{cm} \\
\text { (Normal <1 E-6 S/cm) }\end{array}$ & $\begin{array}{l}<1 \text { E.6 S/cm } \\
\text { Process Design Value. }\end{array}$ \\
\hline II & $\begin{array}{r}-60 C 232 \\
(88984)\end{array}$ & $\begin{array}{l}\text { Effluent pH } \\
\text { Adjustment Tank } \\
\text { Pump (A) Curnent }\end{array}$ & FLA: $26.4 \mathrm{~A}$ & $\begin{array}{l}\text { V-1358-002-002 } \\
\text { FLA: Full Load Amperage }\end{array}$ \\
\hline II & $\begin{array}{r}-60 C 234 \\
(88984)\end{array}$ & $\begin{array}{l}\text { Effluent pH } \\
\text { Adjustment Tank } \\
\text { Pump (B) Current }\end{array}$ & FLA : $26.4 \mathrm{~A}$ & $\begin{array}{l}\text { V-1358-002-002 } \\
\text { FLA: Full Load Amperage }\end{array}$ \\
\hline
\end{tabular}


TABLE 6 60D UV OXIDATION $\left(\mathrm{H}_{2} \mathrm{O}_{2}\right.$ Decomposer)

\begin{tabular}{|c|c|c|c|}
\hline $\begin{array}{l}\text { Tag No. (PID No. } \\
\text { H-2-89337) }\end{array}$ & Name & Set-Values and Functions & Remarks \\
\hline $\begin{array}{lr}\text { AI } & -60 \mathrm{DOO2} \\
\text { (TOC) } & (89334)\end{array}$ & $\begin{array}{l}\text { 1st RO Feed Tank } \\
\text { Inlet TOC }\end{array}$ & Operating : $<10$ & $\begin{array}{l}\text { Design Value. } \\
\text { V-135A-1131-103 }\end{array}$ \\
\hline $\begin{array}{l}\text { AI }-600004 \\
\text { (TOC) } \quad(88977)\end{array}$ & $\begin{array}{l}\text { UV Oxidizer Outlet } \\
\text { TOC }\end{array}$ & $\begin{array}{l}\text { Operating : }<10 \mathrm{ppm} \\
\text { AH: } 10 \mathrm{ppm} \quad \text { Alarm }\end{array}$ & $\begin{array}{l}\text { Design Value. } \\
\text { V-135A-1131-103 }\end{array}$ \\
\hline $\begin{array}{lr}\text { AI } & -60 \mathrm{DO05} \\
\left(\mathrm{H}_{2} \mathrm{O}_{2}\right) & (89332) \\
\end{array}$ & $\begin{array}{l}\mathrm{H}_{2} \mathrm{O}_{2} \text { Decomposer } \\
\text { Outlet } \mathrm{H}_{2} \mathrm{O}_{2}\end{array}$ & Operating : $<0.5 \mathrm{ppm}$ & $\begin{array}{l}\text { Design Value. } \\
\text { V-135A-1131-103 }\end{array}$ \\
\hline $\begin{array}{r}-600007 \\
(89332)\end{array}$ & $\begin{array}{l}\text { Fine Filter Feed } \\
\text { Flow }\end{array}$ & Operating Range : $\mathbf{4 0}-174 \mathrm{gpm}$ & Design Process Value. \\
\hline
\end{tabular}


TABLE 6.1 60D UV OXIDATION (UV Oxidizer-1)

\begin{tabular}{|c|c|c|c|}
\hline $\begin{array}{l}\text { Tag No. (PID No. } \\
\text { H-2-89337) }\end{array}$ & Name & Set-Values and Functions & Remarks \\
\hline $\begin{array}{r}-60 \mathrm{D} 103 \\
(88976)\end{array}$ & $\begin{array}{l}\text { UV Oxidizer-1 } \\
\text { Inlet Pressure }\end{array}$ & $\begin{array}{l}\text { Operating : <15 psig } \\
\text { AHH: } 22 \text { psig * Alarm UV lamp off } \\
\text { AH: } 15 \text { psig Alarm }\end{array}$ & $\begin{array}{l}\text { V-135A-002-004 } \\
\left({ }^{*}\right) \text { Set at PLC }\end{array}$ \\
\hline $\begin{array}{r}-60 \mathrm{D} 104 \\
(88976) \\
\end{array}$ & $\begin{array}{l}\text { UV Oxidizer-1 } \\
\text { Inlet Flow }\end{array}$ & $\begin{array}{l}\text { SV: } 0 \sim 86 \mathrm{gpm} \\
\text { AHH: } 60 \mathrm{gpm}(*) \text { Alarm - Return to "READY" } \\
\text { AH : } 48 \mathrm{gpm}(*) \text { Alarm message } \\
\text { AL: } 18 \mathrm{gpm}(*) \text { Alarm message } \\
\text { ALL: } 17 \mathrm{gpm}(* *) \text { Alarm - UV lamp off }\end{array}$ & $\begin{array}{l}\left({ }^{*}\right) \text { These set points are per chamber. ( } 2 \text { chambers } \\
\text { in UV Oxidizer) } \\
\left({ }^{* *}\right) \text { Set at PLC }\end{array}$ \\
\hline $\begin{array}{r}-60 \mathrm{D} 105 \\
(88976) \\
\end{array}$ & $\begin{array}{l}\text { UV Oxidizer 1A } \\
\text { Outlet Temperature }\end{array}$ & $\begin{array}{l}\text { Operating : } 100 \sim 140 \mathrm{~F} \\
\text { AHH: } 150^{\circ} \mathrm{F}\left({ }^{*}\right) \text { Alarm }- \text { Shut down UV lamps } \\
\text { AH: } 145^{\circ} \mathrm{F} \quad \text { Alarm message }\end{array}$ & $\begin{array}{l}\text { (*) Common alarm of followings: } \\
\text { TI-60D105, TS-60D112, TS-60D113 } \\
\text { Set at PLC }\end{array}$ \\
\hline $\begin{array}{r}-60 \mathrm{D} 106 \\
(88976) \\
\end{array}$ & $\begin{array}{l}\text { UV Oxidizer 1B } \\
\text { Outlet temperature }\end{array}$ & $\begin{array}{l}\text { Operating : } 100 \sim 140 \mathrm{~F} \\
\text { AHH: } 150^{\circ} \mathrm{F}\left({ }^{*}\right) \text { Alarm - Shut down UV lamps } \\
\mathrm{AH}: 145^{\circ} \mathrm{F} \quad \text { Alarm message }\end{array}$ & $\begin{array}{l}(*) \text { Common alarm of followings: } \\
\text { TI-60D106, TS-60D141, TS-60D142 } \\
\text { Set at PLC }\end{array}$ \\
\hline $\begin{array}{lr}\text { AS } & -60 \mathrm{D} 107 \\
\text { (MOIST) } & (88976) \\
\end{array}$ & $\begin{array}{l}\text { UV Oxidizer Outlet } \\
\text { Moisture }\end{array}$ & AH : On $\begin{array}{c}\text { Alarm } \\
\text { Shut down UV lamps }\end{array}$ & $\begin{array}{l}\text { Rupture disk break detector } \\
\text { V-135A-002-004-4 }\end{array}$ \\
\hline $\begin{array}{r}-60 \mathrm{D} 109 \\
(88976) \\
\end{array}$ & $\begin{array}{l}\text { UV Oxidizer 1A } \\
\text { Flow Reverser IA } \\
\text { Pressure } \\
\end{array}$ & $\begin{array}{l}\text { Operating : } 90 \text { psig } \\
\text { AL: } 76 \text { psig Alarm }\end{array}$ & $\begin{array}{l}\text { V-135A-002-004-4 } \\
\text { Ensure Adequate Air for Valve Operation. }\end{array}$ \\
\hline $\begin{array}{r}-60 \mathrm{D} 112 \\
(88976) \\
\end{array}$ & $\begin{array}{l}\text { UV Oxidizer 1A } \\
\text { Inlet Temperature }\end{array}$ & $\begin{array}{l}\text { Operating : }<100^{\circ} \mathrm{F} \\
\text { AH: } 150^{\circ} \mathrm{F} \quad \text { Alarm }\end{array}$ & $\begin{array}{l}\text { V-135A-002-004-4 } \\
\text { Prevent Damage from High Temperature. }\end{array}$ \\
\hline $\begin{array}{r}-60 \mathrm{D} 113 \\
(88976) \\
\end{array}$ & $\begin{array}{l}\text { UV Oxidizer } 1 \mathrm{~A} \\
\text { Inlet Temperature }\end{array}$ & $\begin{array}{l}\text { Operating : }<100^{\circ} \mathrm{F} \\
\mathrm{AH}: 150^{\circ} \mathrm{F} \quad \text { Alarm }\end{array}$ & $\begin{array}{l}\text { Used when reverse flow } \\
\text { V-135A-002-004-4 } \\
\end{array}$ \\
\hline $\begin{array}{lr}\text { AS } & -60 \mathrm{D} 121 \\
\text { (MOIST) } & (88976) \\
\end{array}$ & $\begin{array}{l}\text { UV Oxidizer 1A } \\
\text { Lamp Enclosure } \\
\text { Moisture }\end{array}$ & AH: On $\begin{array}{c}\text { Alarm } \\
\text { Shut down UV lamps }\end{array}$ & $\begin{array}{l}\text { V-135A-002-004-4 } \\
\text { Leak Detection. }\end{array}$ \\
\hline $\begin{array}{r}-60 \mathrm{D} 122 \\
(88976) \\
\end{array}$ & $\begin{array}{l}\text { UV Oxidizer 1A } \\
\text { Lamp Enclosure } \\
\text { Temperature }\end{array}$ & $\begin{array}{c}\text { AH: } 150^{\circ} \mathrm{F} \text { Alarm } \\
\text { Shut down UV lamps }\end{array}$ & $\begin{array}{l}\text { V-135A-002-004-4 } \\
\text { Prevent Damage from High Temperature. }\end{array}$ \\
\hline $\begin{array}{lr}\text { AS } & -60 \mathrm{D} 123 \\
\text { (MOIST) } & (88976)\end{array}$ & $\begin{array}{l}\text { UV Oxidizer 1B } \\
\text { Lamp Enclosure } \\
\text { Moisture }\end{array}$ & $\begin{array}{l}\text { Alarm } \\
\text { Shut down UV lamps }\end{array}$ & $\begin{array}{l}\text { V-135A-002-004-4 } \\
\text { Leak Detection. }\end{array}$ \\
\hline
\end{tabular}


TABLE 6.1 60D UV OXIDATION (UV Oxidizer-1)

\begin{tabular}{|c|c|c|c|}
\hline $\begin{array}{l}\text { Tag No. (PID No. } \\
\text { H-2-89337) }\end{array}$ & \multirow{2}{*}{$\begin{array}{l}\text { Name } \\
\text { UV Oxidizer 1B } \\
\text { Lamp Enclosure } \\
\text { Temperature }\end{array}$} & Set-Values and Functions & \multirow{2}{*}{$\begin{array}{l}\text { Remarks } \\
\text { V-135A-002-004-4 } \\
\text { Prevent Damage from High Temperature. }\end{array}$} \\
\hline $\begin{array}{l}-60 \mathrm{D} 124 \\
(88976) \\
\end{array}$ & & $\begin{array}{ll}\text { AH: } 150^{\circ} \mathrm{F} & \begin{array}{l}\text { Alarm } \\
\text { Shut down UV lamps }\end{array}\end{array}$ & \\
\hline $\begin{array}{r}-60 \mathrm{D} 133 \\
(88976) \\
\end{array}$ & $\begin{array}{l}\text { UV Oxidizer 1B } \\
\text { Flow Reverser IA } \\
\text { Pressure } \\
\end{array}$ & $\begin{array}{l}\text { Operating : } 90 \text { psig } \\
\text { AL: } 76 \text { psig Alarm }\end{array}$ & $\begin{array}{l}\text { V-135A-002-004-4 } \\
\text { Ensure Adequate Air for Valve Operation. }\end{array}$ \\
\hline $\begin{array}{r}-60 \mathrm{D} 141 \\
(88976) \\
\end{array}$ & $\begin{array}{l}\text { UV Oxidizer 1B } \\
\text { Inlet Temperature }\end{array}$ & $\begin{array}{l}\text { Operating : }<100^{\circ} \mathrm{F} \\
\text { AH: } 150^{\circ} \mathrm{F} \quad \text { Alarm } \\
\end{array}$ & $\begin{array}{l}\text { V-135A-002-004-4 } \\
\text { Prevent Damage from High Temperature. }\end{array}$ \\
\hline $\begin{array}{r}-60 \mathrm{D} 142 \\
(88976) \\
\end{array}$ & $\begin{array}{l}\text { UV Oxidizer 1B } \\
\text { Inlet Temperature }\end{array}$ & $\begin{array}{l}\text { Operating : }<100^{\circ} \mathrm{F} \\
\text { AH: } 150^{\circ} \mathrm{F} \quad \text { Alarm } \\
\end{array}$ & $\begin{array}{l}\text { Used when reverse flow } \\
\text { V-135A-002-004 }\end{array}$ \\
\hline $\begin{array}{r}-60 D 151 \\
(88976) \\
\end{array}$ & $\begin{array}{l}\text { UV Oxidizer 1A } \\
\text { Lamp End Door }\end{array}$ & Open Alarm & $V-135 A-002-004$ \\
\hline $\begin{array}{r}-600152 \\
(88976) \\
\end{array}$ & $\begin{array}{l}\text { UV Oxidizer 1B } \\
\text { Lamp End Door }\end{array}$ & Open Alarm & $V-135 A-002-004$ \\
\hline $\begin{array}{r}-600160 \\
(88976) \\
\end{array}$ & $\begin{array}{l}\text { UV Oxidizer } 1 \\
\text { Inlet Temperature }\end{array}$ & $\begin{array}{l}\text { Operating : }<100^{\circ} \mathrm{F} \\
\text { AH: } 150^{\circ} \mathrm{F} \quad \text { Alarm }- \text { Shut down UV lamps }\end{array}$ & $\begin{array}{l}\text { Set at PLC } \\
\text { Prevent Damage from High Temperature }\end{array}$ \\
\hline $\begin{array}{r}-60 D 161 \\
(88976) \\
\end{array}$ & $\begin{array}{l}\text { UV Oxidizer } 1 \mathrm{~A} \\
\text { Level }\end{array}$ & AL: Off & $\begin{array}{l}\text { Detect vaporization } \\
\text { Starting condition of UV lamps. }\end{array}$ \\
\hline $\begin{array}{r}-60 D 164 \\
(88976) \\
\end{array}$ & $\begin{array}{l}\text { UV Oxidizer 1B } \\
\text { Level }\end{array}$ & AL: Off & $\begin{array}{l}\text { Detect vaporization } \\
\text { Starting condition of UV lamps. }\end{array}$ \\
\hline $\begin{array}{r}-60 \mathrm{D} 176 \\
(88976) \\
\end{array}$ & $\begin{array}{l}\text { UV Oxidizer 1A } \\
\text { Lamp Drive Door }\end{array}$ & \begin{tabular}{|l} 
Open Alarm \\
\end{tabular} & $V-135 A-002-004$ \\
\hline $\begin{array}{r}-60 \mathrm{D} 177 \\
(88976) \\
\end{array}$ & $\begin{array}{l}\text { UV Oxidizer 1B } \\
\text { Lamp Drive Door }\end{array}$ & Open Alarm Shut down UV lamps & V-135A-002-004-4 \\
\hline $\begin{array}{r}-60 D 181 \\
6(88976) \\
\end{array}$ & $\begin{array}{l}\text { UV Oxidizer } 1 \mathrm{~A} \\
\text { Lamp Current }\end{array}$ & $\begin{array}{l}\text { Operating : } 18 \sim 27 \mathrm{~A} \\
\mathrm{AL}: 4 \mathrm{~A}(*) \quad \text { Alarm }\end{array}$ & $\begin{array}{l}\text { V-135A-002-004-4 } \\
\text { (*) One common alarm }\end{array}$ \\
\hline $\begin{array}{r}-60 D 187 \\
2(88976) \\
\end{array}$ & $\begin{array}{l}\text { UV Oxidizer 1B } \\
\text { Lamp Current }\end{array}$ & $\begin{array}{l}\text { Operating : } 18 \sim 27 \text { A } \\
\text { AL: } 4 \text { A (*) } \quad \text { Alarm }\end{array}$ & $\begin{array}{l}\text { V-135A-002-004-4 } \\
\text { (*) One common alarm }\end{array}$ \\
\hline $\begin{array}{r}-60 D 193 \\
(88976)\end{array}$ & $\begin{array}{l}\text { UV Oxidizer } 1 A \\
\text { Primary Ground } \\
\text { Fault }\end{array}$ & $\begin{array}{l}\text { Alarm } \\
\text { Shut down UV lamps }\end{array}$ & $\begin{array}{l}\text { V-135A-002-004-4 } \\
\text { Fault Protection }\end{array}$ \\
\hline
\end{tabular}


TABLE 6.1 60D UV OXIDATION (UV Oxidizer-1)

\begin{tabular}{|c|c|c|c|c|c|}
\hline \multicolumn{2}{|c|}{$\begin{array}{l}\text { Tag No. (PID No. } \\
\text { H-2-89337) }\end{array}$} & \multirow{2}{*}{$\begin{array}{l}\text { Name } \\
\text { UV Oxidizer 1A } \\
\text { Secondary Ground } \\
\text { Fault } \\
\end{array}$} & \multicolumn{2}{|c|}{ Set-Values and Functions } & \multirow{2}{*}{$\begin{array}{l}\text { Remarks } \\
\begin{array}{l}\text { V-135A-002-004-4 } \\
\text { Fault Protection }\end{array}\end{array}$} \\
\hline IS & $\begin{array}{r}-60 \mathrm{D} 194 \\
(88976)\end{array}$ & & AH: 4 A & $\begin{array}{l}\text { Alarm } \\
\text { Shut down UV lamps }\end{array}$ & \\
\hline IS & $\begin{array}{r}-60 D 195 \\
(88976)\end{array}$ & $\begin{array}{l}\text { UV Oxidizer 1B } \\
\text { Primary Ground } \\
\text { Fault }\end{array}$ & AH: 4 A & $\begin{array}{l}\text { Alarm } \\
\text { Shut down UV lamps }\end{array}$ & $\begin{array}{l}\text { V-135A-002-004-4 } \\
\text { Fault Protection }\end{array}$ \\
\hline IS & $\begin{array}{r}-60 D 196 \\
(88976)\end{array}$ & $\begin{array}{l}\text { UV Oxidizer 1B } \\
\text { Secondary Ground } \\
\text { Fault }\end{array}$ & $\mathrm{AH}: 4 \mathrm{~A}$ & $\begin{array}{l}\text { Alarm } \\
\text { Shut down UV lamps }\end{array}$ & $\begin{array}{l}\text { V-135A-002-004-4 } \\
\text { Fault Protection }\end{array}$ \\
\hline
\end{tabular}


TABLE 6.2 60D UV OXIDATION (UV Oxidizer 2)

\begin{tabular}{|c|c|c|c|}
\hline $\begin{array}{l}\text { Tag No. (PID No. } \\
\text { H-2-89337) }\end{array}$ & Name & Set-Values and Functions & Remarks \\
\hline $\begin{array}{r}-60 \mathrm{D} 203 \\
(89342) \\
\end{array}$ & $\begin{array}{l}\text { UV Oxidizer-2 } \\
\text { Inlet Pressure }\end{array}$ & $\begin{array}{l}\text { Operating : < } 15 \text { psig } \\
\text { AHH: } 22 \text { psig (*) Alarm } \\
\text { UV lamp off } \\
\text { AH: } 15 \text { psig Alarm }\end{array}$ & $\begin{array}{l}\text { V-135A-002-004 } \\
\left({ }^{*}\right) \text { Set at PLC }\end{array}$ \\
\hline $\begin{array}{r}-60 \mathrm{D} 204 \\
(89342) \\
\end{array}$ & $\begin{array}{l}\text { UV Oxidizer-2 } \\
\text { Inlet Flow }\end{array}$ & $\begin{array}{l}\text { SV: } 0-86 \mathrm{gpm} \\
\text { AHH: } 60 \mathrm{gpm}\left({ }^{*}\right) \text { Alarm - Return to "READY" } \\
\text { AH : } 48 \mathrm{gpm}\left({ }^{*}\right) \text { Alarm message } \\
\text { AL: } 18 \mathrm{gpm}\left({ }^{*}\right) \text { Alarm message } \\
\text { ALL: } 17 \mathrm{gpm}\left({ }^{* *}\right) \text { Alarm - UV lamp off }\end{array}$ & $\begin{array}{l}(*) \text { These set points are per chamber. ( } 2 \text { chambers } \\
\text { in UV Oxidizer) } \\
(* *) \text { Low Low flow set point will be calculated } \\
\text { from \# of Active lamps and TT-60D260 } \\
(* *) \text { Set at PLC }\end{array}$ \\
\hline $\begin{array}{r}-60 \mathrm{D} 205 \\
(89342) \\
\end{array}$ & $\begin{array}{l}\text { UV Oxidizer 2A } \\
\text { Outlet Temperature }\end{array}$ & $\begin{array}{l}\text { Operating : } 100 \sim 140^{\circ} \mathrm{F} \\
\text { AHH: } 150^{\circ} \mathrm{F}(*) \text { Alarm - Shut down UV lamps } \\
\text { AH : } 145^{\circ} \mathrm{F} \quad \text { Alarm message }\end{array}$ & $\begin{array}{l}\text { (*) Common alarm of following: } \\
\text { TI-60D205, TS-60D212, TS-60D213 } \\
\text { Set at PLC }\end{array}$ \\
\hline $\begin{array}{r}-60 \mathrm{D} 206 \\
(89342) \\
\end{array}$ & $\begin{array}{l}\text { UV Oxidizer 2B } \\
\text { Outlet temperature }\end{array}$ & $\begin{array}{l}\text { Operating : } 100-140^{\circ} \mathrm{F} \\
\text { AHH: } 150^{\circ} \mathrm{F}\left({ }^{*}\right) \text { Alarm - Shut down UV lamps } \\
\text { AH: } 145^{\circ} \mathrm{F} \quad \text { Alarm message }\end{array}$ & $\begin{array}{l}(*) \text { Common alarm of followings: } \\
\text { TI-60D206, TS-60D241, TS-60D242 } \\
\text { Set at PLC }\end{array}$ \\
\hline $\begin{array}{lr}\text { AS } & -60 \mathrm{D} 207 \\
\text { (MOIST) } & (89342) \\
\end{array}$ & $\begin{array}{l}\text { UV Oxidizer } 2 \\
\text { Outlet Moisture } \\
\end{array}$ & Alarm - Shut down UV lamps & Rupture disk break detector \\
\hline $\begin{array}{r}-60 \mathrm{D} 209 \\
(89342)\end{array}$ & $\begin{array}{l}\text { UV Oxidizer } 2 A \\
\text { Flow Reverser IA } \\
\text { Pressure }\end{array}$ & $\begin{array}{l}\text { Operating : } 90 \mathrm{psig} \\
\text { AL: } 76 \mathrm{psig} \quad \text { Alarm }\end{array}$ & $\begin{array}{l}\text { V-135A-002-004-4 } \\
\text { Ensure Adequate Air for Valve Operation. }\end{array}$ \\
\hline $\begin{array}{r}-60 \mathrm{D} 212 \\
(89342) \\
\end{array}$ & $\begin{array}{l}\text { UV Oxidizer } 2 \mathrm{~A} \\
\text { Inlet Temperature }\end{array}$ & $\begin{array}{l}\text { Operating : }<100 \mathrm{~F} \\
\text { AH: } 150^{\circ} \mathrm{F} \quad \text { Alarm }\end{array}$ & $\begin{array}{l}\text { V-135A-002-004-4 } \\
\text { Prevent Damage from High Temperature. }\end{array}$ \\
\hline $\begin{array}{lr}\text { TS } & -60 \mathrm{D} 213 \\
(\text { Local }) & (89342) \\
\end{array}$ & $\begin{array}{l}\text { UV Oxidizer } 2 \mathrm{~A} \\
\text { Inlet Temperature }\end{array}$ & $\begin{array}{l}\text { Operating : }<100^{\circ} \mathrm{F} \\
\text { AH: } 150^{\circ} \mathrm{F} \quad \text { Alarm }\end{array}$ & $\begin{array}{l}\text { Used when reverse flow } \\
\text { V-135A-002-004-4 }\end{array}$ \\
\hline $\begin{array}{lr}\text { AS } & -60 D 221 \\
\text { (MOIST) } & (89342)\end{array}$ & $\begin{array}{l}\text { UV Oxidizer 2A } \\
\text { Lamp Enclosure } \\
\text { Moisture }\end{array}$ & $\begin{array}{l}\text { Alarm } \\
\text { Shut down UV lamps }\end{array}$ & $\begin{array}{l}\text { V-135A-002-004-4 } \\
\text { Leak Detection. }\end{array}$ \\
\hline $\begin{array}{r}-60 \mathrm{D} 222 \\
(89342)\end{array}$ & $\begin{array}{l}\text { UV Oxidizer 2A } \\
\text { Lamp Enclosure } \\
\text { Temperature }\end{array}$ & $\begin{array}{ll}\text { AH: } 150^{\circ} \mathrm{F} & \begin{array}{l}\text { Alarm } \\
\text { Shut down UV lamps }\end{array}\end{array}$ & $\begin{array}{l}\text { V-135A-002-004-4 } \\
\text { Prevent Damage from High Temperature. }\end{array}$ \\
\hline $\begin{array}{lr}\text { AS } & -60 D 223 \\
\text { (MOIST) } & (89342)\end{array}$ & $\begin{array}{l}\text { UV Oxidizer 2B } \\
\text { Lamp Enclosure } \\
\text { Moisture }\end{array}$ & $\begin{array}{l}\text { Alarm } \\
\text { Shut down UV lamps }\end{array}$ & $\begin{array}{l}\text { V-135A-002-004-4 } \\
\text { Leak Detection }\end{array}$ \\
\hline
\end{tabular}


TABLE 6.2 60D UV OXIDATION (UV Oxidizer 2)

\begin{tabular}{|c|c|c|c|}
\hline $\begin{array}{l}\text { Tag No. (PID No. } \\
\text { H-2-89337) }\end{array}$ & \multirow{2}{*}{$\begin{array}{l}\text { Name } \\
\begin{array}{l}\text { UV Oxidizer 2B } \\
\text { Lamp Enclosure } \\
\text { Temperature }\end{array} \\
\end{array}$} & Set-Values and Functions & \multirow{2}{*}{$\begin{array}{l}\text { Remarks } \\
\text { V-135A-002-004-4 } \\
\text { Prevent Damage from High Temperature. }\end{array}$} \\
\hline $\begin{array}{r}-60 \mathrm{D} 224 \\
(89342)\end{array}$ & & $\begin{array}{ll}\text { AH: } 150^{\circ} \mathrm{F} & \text { Alarm } \\
& \text { Shut down UV lamps }\end{array}$ & \\
\hline $\begin{array}{r}-60 \mathrm{D} 233 \\
(89342)\end{array}$ & \begin{tabular}{|l} 
UV Oxidizer 2B \\
Flow Reverser IA \\
Pressure \\
\end{tabular} & $\begin{array}{l}\text { Operating : } 90 \text { psig } \\
\text { AL: } 76 \text { psig Alarm }\end{array}$ & $\begin{array}{l}\text { V-135A-002-004-4 } \\
\text { Ensure Adequate Air for Valve Operation. }\end{array}$ \\
\hline $\begin{array}{r}-60 \mathrm{D} 241 \\
(89342) \\
\end{array}$ & $\begin{array}{l}\text { UV Oxidizer 2B } \\
\text { Inlet Temperature }\end{array}$ & $\begin{array}{l}\text { Operating : }<100^{\circ} \mathrm{F} \\
\text { AH: } 150^{\circ} \mathrm{F} \quad \text { Alarm }\end{array}$ & $\begin{array}{l}\text { V-135A-002-004-4 } \\
\text { Prevent Damage from High Temperature. }\end{array}$ \\
\hline $\begin{array}{r}-60 \mathrm{D} 242 \\
(89342) \\
\end{array}$ & $\begin{array}{l}\text { UV Oxidizer 2B } \\
\text { Inlet Temperature }\end{array}$ & $\begin{array}{l}\text { Operating : }<100^{\circ} \mathrm{F} \\
\mathrm{AH}: \quad 150^{\circ} \mathrm{F} \quad \text { Alarm }\end{array}$ & $\begin{array}{l}\text { Used when reverse flow } \\
\text { V-135A-002-004-4 }\end{array}$ \\
\hline $\begin{array}{r}-60 D 251 \\
(89342) \\
\end{array}$ & $\begin{array}{l}\text { UV Oxidizer } 2 \mathrm{~A} \\
\text { Lamp End Door }\end{array}$ & Alarm & $V-135 A-002-004-4$ \\
\hline $\begin{array}{r}-60 \mathrm{D} 252 \\
(89342) \\
\end{array}$ & $\begin{array}{l}\text { UV Oxidizer 2B } \\
\text { Lamp End Door }\end{array}$ & Alarm & V-135A-002-004-4 \\
\hline $\begin{array}{r}-60 \mathrm{D} 260 \\
(89342)\end{array}$ & $\begin{array}{l}\text { UV Oxidizer } 2 \\
\text { Inlet Temperature }\end{array}$ & $\begin{array}{c}\text { Operating : }<100^{\circ} \mathrm{F} \\
\text { AH: } 150^{\circ} \mathrm{F} \text { Alarm } \\
\text { Shut down UV lamps }\end{array}$ & $\begin{array}{l}\text { Set at PLC } \\
\text { Prevent Damage from High Temperature. }\end{array}$ \\
\hline $\begin{array}{r}-60 \mathrm{D} 261 \\
(89342) \\
\end{array}$ & $\begin{array}{l}\text { UV Oxidizer } 2 \mathrm{~A} \\
\text { Level }\end{array}$ & AL: OFF & $\begin{array}{l}\text { Detect vaporization } \\
\text { Starting condition of UV lamps. }\end{array}$ \\
\hline $\begin{array}{r}-600264 \\
(89342) \\
\end{array}$ & $\begin{array}{l}\text { UV Oxidizer 2B } \\
\text { Level }\end{array}$ & AL: OFF & $\begin{array}{l}\text { Detect vaporization } \\
\text { Starting condition of UV lamps. }\end{array}$ \\
\hline $\begin{array}{r}-60 D 276 \\
(89342) \\
\end{array}$ & $\begin{array}{l}\text { UV Oxidizer } 2 \mathrm{~A} \\
\text { Lamp Drive Door }\end{array}$ & $\begin{array}{l}\text { Alarm } \\
\text { Shut down UV lamps }\end{array}$ & $V-135 A-002-004-4$ \\
\hline $\begin{array}{r}-60 \mathrm{D} 277 \\
(89342) \\
\end{array}$ & $\begin{array}{l}\text { UV Oxidizer 2B } \\
\text { Lamp Drive Door }\end{array}$ & $\begin{array}{l}\text { Alarm } \\
\text { Shut down UV lamps }\end{array}$ & $V-135 A-002-004-4$ \\
\hline $\begin{array}{l}-60 \mathrm{D} 281 \\
86)\end{array}$ & $\begin{array}{l}\text { UV Oxidizer } 2 \mathrm{~A} \\
\text { Lamp Current }\end{array}$ & 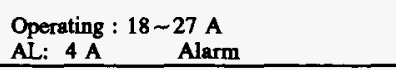 & $V-135 A-002-004-4$ \\
\hline $\begin{array}{l}-60 D 287 \\
92)\end{array}$ & $\begin{array}{l}\text { UV Oxidizer 2B } \\
\text { Lamp Current }\end{array}$ & $\begin{array}{l}\text { Operating : } 18 \sim 27 \mathrm{~A} \\
\text { AL: } 4 \mathrm{~A} \quad \text { Alarm }\end{array}$ & $V-135 A-002-004-4$ \\
\hline
\end{tabular}


TABLE 6.2 60D UV OXIDATION (UV Oxidizer 2)

\begin{tabular}{|c|c|c|c|c|}
\hline $\begin{array}{l}\text { Tag No. (PID No. } \\
\text { H-2-89337) }\end{array}$ & \multirow{2}{*}{$\begin{array}{l}\text { Name } \\
\text { UV Oxidizer 2A } \\
\text { Primary Ground } \\
\text { Fault }\end{array}$} & \multicolumn{2}{|c|}{ Set-Values and Functions } & \multirow{2}{*}{$\begin{array}{l}\text { Remarks } \\
\text { V-135A-002-004-4 } \\
\text { Fault Protection. }\end{array}$} \\
\hline $\begin{array}{r}-60 D 293 \\
(89342) \\
\end{array}$ & & AH: $4 \mathrm{~A}$ & $\begin{array}{l}\text { Alarm } \\
\text { Shut down UV lamps }\end{array}$ & \\
\hline $\begin{array}{r}-60 D 294 \\
(89342) \\
\end{array}$ & $\begin{array}{l}\text { UV Oxidizer } 2 \mathrm{~A} \\
\text { Secondary Ground } \\
\text { Fault }\end{array}$ & $\mathrm{AH}: 4 \mathrm{~A}$ & $\begin{array}{l}\text { Alarm } \\
\text { Shut down UV lamps }\end{array}$ & $\begin{array}{l}\text { V-135A-002-004-4 } \\
\text { Fault Protection. }\end{array}$ \\
\hline $\begin{array}{r}-60 \mathrm{D} 295 \\
(89342) \\
\end{array}$ & $\begin{array}{l}\text { UV Oxidizer 2B } \\
\text { Primary Ground } \\
\text { Fault }\end{array}$ & AH: $4 \mathrm{~A}$ & $\begin{array}{l}\text { Alarm } \\
\text { Shut down UV lamps }\end{array}$ & $\begin{array}{l}\text { V-135A-002-004-4 } \\
\text { Fault Protection. }\end{array}$ \\
\hline $\begin{array}{r}-600296 \\
(89342)\end{array}$ & $\begin{array}{l}\text { UV Oxidizer 2B } \\
\text { Secondary Ground } \\
\text { Fault }\end{array}$ & AH: 4 A & $\begin{array}{l}\text { Alarm } \\
\text { Shut down UV lamps }\end{array}$ & $\begin{array}{l}\text { V-135A-002-004-4 } \\
\text { Fault Protection. }\end{array}$ \\
\hline
\end{tabular}


TABLE 6.3 60D UV OXIDATION $\left(\mathrm{H}_{2} \mathrm{O}_{2}\right.$ Feed System)

\begin{tabular}{|c|c|c|c|}
\hline $\begin{array}{l}\text { Tag No. (PID No. } \\
\text { H-2-89337) }\end{array}$ & Name & Set-Values and Functions & Remarks \\
\hline $\begin{array}{r}-60 \mathrm{D} 301 \\
(89350) \\
\end{array}$ & $\begin{array}{l}\mathrm{H}_{2} \mathrm{O}_{2} \text { Feed Pump } \\
1 \mathrm{A1} \text { Discharge } \\
\text { Pressure }\end{array}$ & $\begin{array}{l}\text { Operating : }<60 \text { psig } \\
\text { AH: } 55 \text { psig Alarm } \\
\text { AL: } 15 \text { psig (*) Alarm - Stop } \mathrm{H}_{2} \mathrm{O}_{2} \text { Feed Pump 1A1 }\end{array}$ & $\begin{array}{l}\text { (*) Common alarm of followings: } \\
\text { PI-60D301, PI-60D302 } \\
\text { V-135A-002-810 }\end{array}$ \\
\hline $\begin{array}{r}-60 \mathrm{D} 302 \\
(89350) \\
\end{array}$ & $\begin{array}{l}\mathrm{H}_{2} \mathrm{O}_{2} \text { Feed Pump } \\
1 \mathrm{~A} 2 \text { Discharge } \\
\text { Pressure }\end{array}$ & $\begin{array}{l}\text { Operating : }<60 \text { psig } \\
\text { AH: } 55 \text { psig Alarm } \\
\text { AL: } 15 \text { psig (*) Alarm - Stop } \mathrm{H}_{2} \mathrm{O}_{2} \text { Feed Pump } 1 \mathrm{~A} 2\end{array}$ & See Above. \\
\hline $\begin{array}{r}-60 \mathrm{D} 303 \\
(89350) \\
\end{array}$ & $\begin{array}{l}\mathrm{H}_{2} \mathrm{O}_{2} \text { Feed Pump } \\
1 \mathrm{~B} 1 \text { Discharge } \\
\text { Pressure }\end{array}$ & $\begin{array}{l}\text { Operating : }<60 \text { psig } \\
\text { AH: } 55 \text { psig Alarm } \\
\text { AL: } 15 \text { psig (*) Alarm - Stop } \mathrm{H}_{2} \mathrm{O}_{2} \text { Feed Pump 1B1 }\end{array}$ & $\begin{array}{l}\text { (*) Common alarm of followings: } \\
\text { PI-60D303, PI-60D304 } \\
\text { V-135A-002-810 } \\
\end{array}$ \\
\hline $\begin{array}{r}-60 \mathrm{D} 304 \\
(89350) \\
\end{array}$ & $\begin{array}{l}\mathrm{H}_{2} \mathrm{O}_{2} \text { Feed Pump } \\
1 \mathrm{~B} 2 \text { Discharge } \\
\text { Pressure }\end{array}$ & $\begin{array}{l}\text { Operating : }<60 \text { psig } \\
\text { AH: } 55 \text { psig Alarm } \\
\text { AL: } 15 \text { psig }\left(^{*}\right) \text { Alarm }- \text { Stop } \mathrm{H}_{2} \mathrm{O}_{2} \text { Feed Pump } 1 \mathrm{~B} 2\end{array}$ & See Above. \\
\hline $\begin{array}{r}-60 \mathrm{D} 313 \\
(89350) \\
\end{array}$ & $\begin{array}{l}\mathrm{H}_{2} \mathrm{O}_{2} \text { Feed Tank } \\
\text { Level }\end{array}$ & $\begin{array}{ll}\text { AH: } 95 \% & \text { Alarm } \\
\text { AL: } 25 \% & \text { Alarm - Display " } \mathrm{H}_{2} \mathrm{O}_{2} \text { SUPPLY REQUIRED } \\
\text { ALL: } 7.0 \% & \text { Alarm - Stop } \mathrm{H}_{2} \mathrm{O}_{2} \text { Feed Pump }\end{array}$ & $\begin{array}{l}\text { AL: } 10 \text { days capacity } 47.4 \times 10=474(\mathrm{gal}) \\
\mathrm{H}_{2} \mathrm{O}_{2} \text { Consumption } \\
171(\mathrm{gpm}) \times(200+30)(\mathrm{ppm}) \times 10^{-6} \times(60 \times 24) \\
11.195=47.4(\mathrm{gal} / \mathrm{day})\end{array}$ \\
\hline $\begin{array}{r}-60 \mathrm{D} 336 \\
(89350) \\
\end{array}$ & $\begin{array}{l}\mathrm{H}_{2} \mathrm{O}_{2} \text { Feed Pump } \\
2 \mathrm{~A} 1 \text { Discharge } \\
\text { Pressure }\end{array}$ & $\begin{array}{l}\text { Operating : }<60 \text { psig } \\
\text { AH: } 55 \text { psig Alarm } \\
\text { AL: } 15 \text { psig (*) Alarm - Stop } \mathrm{H}_{2} \mathrm{O}_{2} \text { Feed Pump 2A1 }\end{array}$ & $\begin{array}{l}\text { (*) Common alarm of followings: } \\
\text { PI-60D301, PI-60D302 } \\
\text { V-135A-002-810 }\end{array}$ \\
\hline $\begin{array}{r}-60 D 337 \\
(89350) \\
\end{array}$ & $\begin{array}{l}\mathrm{H}_{2} \mathrm{O}_{2} \text { Feed Pump } \\
2 \mathrm{~A} 2 \text { Discharge } \\
\text { Pressure }\end{array}$ & $\begin{array}{l}\text { Operating : }<60 \text { psig } \\
\text { AH: } 55 \text { psig Alarm } \\
\text { Al: } 15 \text { psig }\left(^{*}\right) \text { Alarm - Stop } \mathrm{H}_{2} \mathrm{O}_{2} \text { Feed Pump } 2 \mathrm{A2}\end{array}$ & See Above. \\
\hline $\begin{array}{r}-60 \mathrm{D} 338 \\
(89350) \\
\end{array}$ & $\begin{array}{l}\mathrm{H}_{2} \mathrm{O}_{2} \text { Feed Pump } \\
2 \mathrm{~B} 1 \text { Discharge } \\
\text { Pressure }\end{array}$ & $\begin{array}{l}\text { Operating : }<60 \text { psig } \\
\text { AH: } 55 \text { psig Alarm } \\
\text { AL: } 15 \text { psig (*) Alarm - Stop } \mathrm{H}_{2} \mathrm{O}_{2} \text { Feed Pump 2B1 }\end{array}$ & $\begin{array}{l}\text { (*) Common alarm of followings: } \\
\text { PI-60D303, PI-60D } 304 \\
\text { V-135A-002-810 }\end{array}$ \\
\hline $\begin{array}{r}-60 D 340 \\
(89350)\end{array}$ & $\begin{array}{l}\mathrm{H}_{2} \mathrm{O}_{2} \text { Feed Pump } \\
\text { 2B2 Discharge } \\
\text { Pressure }\end{array}$ & $\begin{array}{l}\text { Operating : <60 psig } \\
\text { AH: } 55 \text { psig (*) Alarm } \\
\text { AL: } 15 \text { psig ( }\left(^{*}\right) \text { Alarm - Stop } \mathrm{H}_{2} \mathrm{O}_{2} \text { Feed Pump 2B2 }\end{array}$ & See Above. \\
\hline
\end{tabular}


TABLE 7 60D UV OXIDATION SYSTEM $\left(\mathrm{H}_{2} \mathrm{O}_{2}\right.$ Decomposer System - by PSI)

\begin{tabular}{|c|c|c|c|}
\hline $\begin{array}{l}\text { Tag No. (PID No. } \\
\text { H-2-89337) }\end{array}$ & Name & Set-Values and Functions & Remarks \\
\hline $\begin{array}{r}-60 D 401 \\
(89332) \\
\end{array}$ & $\begin{array}{l}\mathrm{H}_{2} \mathrm{O}_{2} \text { Decomposer } \\
\text { Inlet Pressure }\end{array}$ & Operating : 100 psig & $\begin{array}{l}\text { V-135A-002-004-4 } \\
\text { System Design. }\end{array}$ \\
\hline $\begin{array}{r}-60 \mathrm{D} 402 \\
(89332) \\
\end{array}$ & $\begin{array}{l}\mathrm{H}_{2} \mathrm{O}_{2} \text { Decomposer } \\
\text { (A) Outlet Pressure }\end{array}$ & Operating : 85 psig & $\begin{array}{l}\text { V-135A-002-004-4 } \\
\text { System Design. }\end{array}$ \\
\hline $\begin{array}{r}-60 \mathrm{D} 403 \\
(89332) \\
\end{array}$ & $\begin{array}{l}\mathrm{H}_{2} \mathrm{O}_{2} \text { Decomposer } \\
\text { (B) Outlet Pressure }\end{array}$ & Operating : 85 psig & $\begin{array}{l}\text { V-135A-002-004-4 } \\
\text { System Design. }\end{array}$ \\
\hline \begin{tabular}{ll|} 
AS & $-60 \mathrm{D} 404$ \\
(MOIST) & $(89332)$ \\
\end{tabular} & $\begin{array}{l}\mathrm{H}_{2} \mathrm{O}_{2} \text { Decomposer } \\
\text { Safety Valve Outlet } \\
\text { Moisture } \\
\end{array}$ & $\begin{array}{ll}\text { AH: ON } & \text { Alarm } \\
& \text { Shut down UV lamps }\end{array}$ & $\begin{array}{l}\text { V-135A-002-004-4 } \\
\text { Senses Relief Valve Discharge to the Sump. }\end{array}$ \\
\hline $\begin{array}{lr}\text { AS } & -60 \mathrm{D} 405 \\
\text { (MOIST) } & (89332)\end{array}$ & $\begin{array}{l}\mathrm{H}_{2} \mathrm{O}_{2} \text { Decomposer } \\
\text { Safety Valve Outlet } \\
\text { Moisture }\end{array}$ & $\begin{array}{cc}\text { AH: ON } & \text { Alarm } \\
& \text { Shut down UV lamps }\end{array}$ & See Above. \\
\hline
\end{tabular}


TABLE $8 \quad$ 60E DEGASIFICATION SYSTEM

\begin{tabular}{|c|c|c|c|c|}
\hline \multicolumn{2}{|c|}{$\begin{array}{l}\text { Tag No. (PID No. } \\
\text { H-2-89337) }\end{array}$} & \multirow{2}{*}{\begin{tabular}{|l} 
Name \\
$\begin{array}{l}\text { Degasification } \\
\text { Column Inlet Cooler } \\
\text { Outlet Temperature }\end{array}$ \\
\end{tabular}} & Set-Values and Functions & \multirow{2}{*}{$\begin{array}{l}\text { Remarks } \\
\begin{array}{l}\text { System Process Design. High Temperature for } \\
\text { Operation. }\end{array}\end{array}$} \\
\hline TIC & $\begin{array}{r}-60 \mathrm{E} 001 \\
(89334)\end{array}$ & & $\begin{array}{l}\text { AH: } 104^{\circ} \mathrm{F} \quad \text { Alarm } \\
\text { SV: } 86^{\circ} \mathrm{F}\end{array}$ & \\
\hline $\begin{array}{l}\text { AI } \\
\text { (COND) }\end{array}$ & $\begin{array}{r}-60 \mathrm{E} 003 \\
(89334) \\
\end{array}$ & $\begin{array}{l}\text { Degasification } \\
\text { Column Outlet } \\
\text { Conductivity }\end{array}$ & Operating : $0 \sim 10,000 \mathrm{E}-6 \mathrm{~s} / \mathrm{cm}$ & System Process Design Value. \\
\hline PI & $\begin{array}{r}-60 \mathrm{E} 010 \\
(89334) \\
\end{array}$ & $\begin{array}{l}\text { Degasification } \\
\text { Column Vent Gas } \\
\text { Pressure } \\
\end{array}$ & $\begin{array}{l}\text { Operating : }-1.8^{n} \text { IN W.C. } \\
\text { AH: } 0.0^{\prime \prime} \text { IN W.C. Alarm }\end{array}$ & $\begin{array}{l}\text { W.C.: Water Column } \\
\text { System Process Design Value. }\end{array}$ \\
\hline LIC & $\begin{array}{r}-60 \mathrm{E} 012 \\
(89334)\end{array}$ & $\begin{array}{l}\text { Degasification } \\
\text { Column Level }\end{array}$ & $\begin{array}{lc}\text { AH: } 90 \% & \text { Alarm - Change RO loop to "SHUTDOWN" } \\
\text { SV: } 50 \% & \\
\text { AL: } 10 \% & \text { Alarm - Stop Degasification Pump } \\
& 2025 \mathrm{E}-60 \mathrm{E}-\mathrm{P}-1 \mathrm{~A}, \mathrm{~B} \\
\end{array}$ & System Process Design Value. \\
\hline $\mathbf{P I}$ & $\begin{array}{r}-60 \mathrm{E} 021 \\
(89334)\end{array}$ & $\begin{array}{l}\text { Degasification Pump } \\
\text { Discharge Pressure }\end{array}$ & Operating : 43 psig (at $175 \mathrm{gpm}$ ) & $\begin{array}{l}\text { System Process Design Value. } \\
\text { Pump Design }\end{array}$ \\
\hline II & $\begin{array}{r}-60 \mathrm{E} 032 \\
(89334) \\
\end{array}$ & $\begin{array}{l}\text { Degasification Pump } \\
\text { (A) Current } \\
\end{array}$ & FLA: $19.4 \mathrm{~A}$ & FLA: Full Load Amperage. \\
\hline II & $\begin{array}{r}-60 \mathrm{E} 034 \\
(89334)\end{array}$ & $\begin{array}{l}\text { Degasification Pump } \\
\text { (B) Current }\end{array}$ & FLA: $19.4 \mathrm{~A}$ & FLA: Full Load Amperage. \\
\hline II & $\begin{array}{r}-60 E 036 \\
(89334)\end{array}$ & $\begin{array}{l}\text { Degasification } \\
\text { Blower Current }\end{array}$ & FLA: $3.9 \mathrm{~A}$ & FLA: Full Load Amperage. \\
\hline
\end{tabular}


TABLE 9 60F REVERSE OSMOSIS (1st RO Feed System)

\begin{tabular}{|c|c|c|c|c|}
\hline \multicolumn{2}{|c|}{$\begin{array}{l}\text { Tag No. (PID No. } \\
\mathrm{H}-2-89337 \text { ) } \\
\end{array}$} & \multirow{2}{*}{$\begin{array}{l}\text { Name } \\
\begin{array}{l}\text { 1st Ro Feed Tank } \\
\text { Level }\end{array}\end{array}$} & Set-Values and Functions & \multirow{2}{*}{$\begin{array}{l}\text { Remarks } \\
\text { See Equipment Process Calculation } \\
\text { Level Control }\end{array}$} \\
\hline LIC & $\begin{array}{r}-60 F 101 \\
(88979) \\
\end{array}$ & & $\begin{array}{ll}\text { AH : } 95 \% & \text { Alarm } \\
\text { SV : } 50 \% & \text { Change RO System loop to "SHUT-DOWN" } \\
\text { AL : } 10 \% & \text { Alarm - Stop 1st RO Feed Tank Pumps } \\
& \text { 2025E-60F-P-1A,B } \\
\end{array}$ & \\
\hline TI & $\begin{array}{l}-60 \mathrm{~F} 102 \\
(88979) \\
\end{array}$ & $\begin{array}{l}\text { 1st RO Feed Tank } \\
\text { Temperature }\end{array}$ & $\begin{array}{l}\text { Operating Range: } 50-86^{\circ} \mathrm{F} \\
\text { AH : } 104^{\circ} \mathrm{F} \text { Alarm }- \text { Stop 1st RO Feed Tank Pump } \\
\text { 2025E-60F-P-1A,B }\end{array}$ & $\begin{array}{l}\text { Influent temperature is set } 86^{\circ} \mathrm{F} \text { by Degasification } \\
\text { Column Inlet Cooler. } \\
\text { Maximum operating temperature : } 113^{\circ} \mathrm{F}\end{array}$ \\
\hline PI & $\begin{array}{r}-60 \mathrm{~F} 111 \\
(88979) \\
\end{array}$ & $\begin{array}{l}\text { 1st RO Feed Tank } \\
\text { Pump Discharge } \\
\text { Pressure }\end{array}$ & $\begin{array}{l}\text { Operating: } 300 \text { psig (at } 200 \mathrm{gpm} \text { ) } \\
\text { AH : } 400 \text { psig Alarm - Stop 1st RO Feed Tank Pump } \\
\text { AL : } 200 \text { psig Alarm }\end{array}$ & $\begin{array}{l}\text { System Process Design Value. } \\
\text { Pump Design. } \\
\text { Prevent Overpressurization of Membranes. }\end{array}$ \\
\hline AI & $\begin{array}{r}-60 \mathrm{~F} 112 \\
(88979) \\
\end{array}$ & 1st RO Inlet pH & 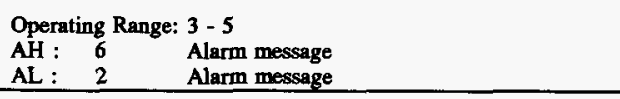 & $\begin{array}{l}\text { pH is set at } 4 \text { by AIC-60C103 } \\
\text { System Process Design Value. }\end{array}$ \\
\hline $\begin{array}{l}\text { AI } \\
\text { (COND) }\end{array}$ & $\begin{array}{l}-60 \mathrm{~F} 113 \\
(88979) \\
\end{array}$ & $\begin{array}{l}\text { 1st RO Inlet } \\
\text { Conductivity }\end{array}$ & $\begin{array}{l}\text { Operating Range: } 0-10,000 \mathrm{E}-6 \mathrm{~s} / \mathrm{cm} \\
\mathrm{AH}: \quad 7000 \mathrm{E}-6 \mathrm{~s} / \mathrm{cm} \quad \text { Alarm message }\end{array}$ & $\begin{array}{l}\text {-Proof of Test Data: } 47,400 \\
\text { (E-6 S/cm) } \\
\text {-Calculated Value: } 7,000(\mathrm{E}-6 \mathrm{~S} / \mathrm{cm})\end{array}$ \\
\hline II & $\begin{array}{r}-60 F 132 \\
(88979) \\
\end{array}$ & $\begin{array}{l}\text { 1st RO Feed Tank } \\
\text { Pump (A) Current }\end{array}$ & FLA: $66 \mathrm{~A}$ & $\begin{array}{l}\text { V-135A-003-049 } \\
\text { FLA: Full load Amperage }\end{array}$ \\
\hline II & $\begin{array}{r}-60 F 134 \\
(88979)\end{array}$ & $\begin{array}{l}\text { 1st RO Feed Tank } \\
\text { Pump (B) Current }\end{array}$ & FLA: $66 \mathrm{~A}$ & $\begin{array}{l}\text { V-135A-003-049 } \\
\text { FLA: Full load Amperage }\end{array}$ \\
\hline
\end{tabular}


TABLE 10 60F REVERSE OSMOSIS (1st RO Stage)

\begin{tabular}{|c|c|c|c|c|}
\hline \multicolumn{2}{|c|}{$\begin{array}{l}\text { Tag No. (PID No. } \\
\text { H-2-89337) }\end{array}$} & \multirow{2}{*}{\begin{tabular}{|l|} 
Name \\
1st RO Inlet Pressure \\
\end{tabular}} & Set-Values and Functions & \multirow[t]{2}{*}{ Remarks } \\
\hline PIC & $\begin{array}{l}-60 \mathrm{~F} 100 \\
(88980)\end{array}$ & & $\begin{array}{l}\text { Operating : } 270 \text { psig } \\
\text { AH : } 400 \text { psi Alarm message - Display "1st RO cleaning } \\
\text { Operation required" } \\
\text { SV : FIC-60F169 }\end{array}$ & \\
\hline PI & $\begin{array}{r}-60 \mathrm{~F} 160 \\
(88980)\end{array}$ & $\begin{array}{l}\text { 1st Array Brine } \\
\text { Pressure }\end{array}$ & $\begin{array}{l}\text { Operating : } 210 \mathrm{psig} \\
\text { AL : } 20 \text { psig Alarm - Stop 1st RO Booster Pump }\end{array}$ & $\begin{array}{l}\text { Prevent 1ST RO Booster Pump from Running Dry. } \\
\text { V-135A-003-052 }\end{array}$ \\
\hline FI & $\begin{array}{r}-60 \mathrm{~F} 161 \\
(88980)\end{array}$ & 1st Array Brine Flow & Operating : $81 \mathrm{gpm}$ & Design Process Value. V-135-003-050 \\
\hline $\begin{array}{l}\mathrm{AI} \\
\text { (COND) }\end{array}$ & $\begin{array}{r}-60 \mathrm{~F} 162 \\
(88980) \\
\end{array}$ & $\begin{array}{l}\text { 1st Array Brine } \\
\text { Conductivity }\end{array}$ & Operating : $11,000 \mathrm{E}-6 \mathrm{~s} / \mathrm{cm}$ & $\begin{array}{l}\text { Design : } 7410 \mathrm{ppm} \\
0-15,000 \mathrm{E}-6 \mathrm{~s} / \mathrm{cm}\end{array}$ \\
\hline II & $\begin{array}{r}-60 \mathrm{~F} 164 \\
(88980)\end{array}$ & $\begin{array}{l}\text { 1st RO Booster Pump } \\
\text { (A) Current }\end{array}$ & FLA : $20.5 \mathrm{~A}$ & FLA: Full load Amperage \\
\hline II & $\begin{array}{r}-60 \mathrm{~F} 166 \\
(88980)\end{array}$ & $\begin{array}{l}\text { 1st RO Booster pump } \\
\text { (B) Current }\end{array}$ & FLA : $20.5 \mathrm{~A}$ & FLA: Full load Amperage \\
\hline PI & $\begin{array}{r}-60 \mathrm{~F} 167 \\
(88980)\end{array}$ & $\begin{array}{l}\text { 1st RO Booster Pump } \\
\text { Discharge Pressure }\end{array}$ & 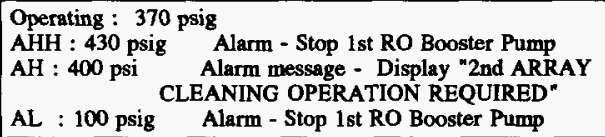 & To protect RO membrane from overpressure \\
\hline PI & $\begin{array}{r}-60 \mathrm{~F} 168 \\
(88980)\end{array}$ & $\begin{array}{l}\text { 1st Array Permeate } \\
\text { Pressure }\end{array}$ & $\begin{array}{lc}\text { Operating : } & 10 \text { psig } \\
\text { AH : } 25 \text { psi } & \text { Alarm message }\end{array}$ & $\begin{array}{l}\text { To protect RO Membrane from excessive } \\
\text { backpressure. V-135A-003-052 }\end{array}$ \\
\hline FIC & $\begin{array}{r}-60 \mathrm{~F} 169 \\
(88980)\end{array}$ & $\begin{array}{l}\text { 1st Array Permeate } \\
\text { Flow }\end{array}$ & $\begin{array}{ll}\text { Operating : } & 171 \mathrm{gpm} \\
\text { SV : } & 171 \mathrm{gpm}\end{array}$ & $\begin{array}{l}\text { PV : PT-60F169+FT-60F188 0-200gpm } \\
\text { CS : To PIC-60F } 100 \\
\text { CS : Control Signal }\end{array}$ \\
\hline $\begin{array}{l}\text { AI } \\
\text { (COND) }\end{array}$ & $\begin{array}{r}-60 F 170 \\
(88980)\end{array}$ & $\begin{array}{l}\text { 1st Array Permeate } \\
\text { Conductivity }\end{array}$ & $\begin{array}{ll}\text { Operating: } & 400 \mathrm{E}-6 \mathrm{~s} / \mathrm{cm} \\
\mathrm{AH}: & 600 \mathrm{E}-6 \mathrm{~s} / \mathrm{cm} \quad \text { Alarm message }\end{array}$ & Design : 223 ppm \\
\hline TI & $\begin{array}{r}-60 \mathrm{~F} 171 \\
(88980)\end{array}$ & $\begin{array}{l}\text { 1st Array Permeate } \\
\text { Temperature }\end{array}$ & \begin{tabular}{|ll} 
Operating : & $90^{\circ} \mathrm{F}$ \\
AH: $113^{\circ} \mathrm{F}$ & Alarm - Stop 1st RO Feed Tank Purm \\
& $2025 \mathrm{E}-60 \mathrm{~F}-\mathrm{P}-1 \mathrm{~A}, \mathrm{~B}$
\end{tabular} & $\begin{array}{l}\text { To protect RO Membrane from High Temperature } \\
\text { V-135A-003-052 }\end{array}$ \\
\hline PI & $\begin{array}{r}-60 \mathrm{~F} 181 \\
(88980)\end{array}$ & $\begin{array}{l}\text { 2nd Array Brine } \\
\text { Pressure }\end{array}$ & Operating : 325 psig & System Design Process Value \\
\hline FIC & $\begin{array}{l}-60 \mathrm{~F} 182 \\
(88980)\end{array}$ & 2nd Array Brine Flow & $\begin{array}{l}\text { Operating : } 15 \mathrm{gpm} \\
\text { SV: (FI-60F169+FI-60F188)X0.09 }\end{array}$ & $\begin{array}{l}\text { Design: FI-60F182 } \quad: 17 \mathrm{gpm} \\
\text { FI-60F169+60F188: } 171 \mathrm{gpm} \\
\text { PV : FT-60F182 } \\
\text { CS : To FY-60F182 }\end{array}$ \\
\hline RI & $\begin{array}{r}-60 \mathrm{~F} 183 \\
(88980)\end{array}$ & 1st RO Brine Activity & Operating : $3 \mathrm{E}-3 \mathrm{Ci} / \mathrm{m}^{3}$ & System Design Process Value \\
\hline
\end{tabular}


TABLE 10 60F REVERSE OSMOSIS (1st RO Stage)

\begin{tabular}{|lr|l|l|l|}
\hline \begin{tabular}{|lrl||} 
Tag No. (PID No. \\
H-2-89337)
\end{tabular} & Name & Set-Values and Functions & Remarks \\
\hline AI & $-60 F 184$ & 1st RO Brine pH & $\begin{array}{l}\text { Operating Range : 0- 14 } \\
\text { Operating }: 4.9 \\
\text { AH: 6.0 Alarm message } \\
\text { AL:4.0 Alarm message }\end{array}$ & $\begin{array}{l}\text { Protect RO Membranes from Damage due to High } \\
\text { or Low pH. }\end{array}$ \\
V-135A-003-052
\end{tabular}


TABLE 11 60F REVERSE OSMOSIS (2nd RO Feed System)

\begin{tabular}{|c|c|c|c|c|}
\hline \multicolumn{2}{|c|}{$\begin{array}{l}\text { Tag No. (PID No. } \\
\text { H-2-89337) }\end{array}$} & \multirow{2}{*}{$\begin{array}{l}\text { Name } \\
\begin{array}{l}\text { 2nd RO Feed Tank } \\
\text { Level }\end{array}\end{array}$} & Set-Values and Functions & \multirow{2}{*}{$\begin{array}{l}\text { Remarks } \\
\text { PV : LT-60F201 } 0-60^{\prime \prime} \mathrm{H}_{2} \mathrm{O} \\
\text { CS : FIC-60F263 } \\
\text { Design flow rate of LCV-60F201 is } 171 \mathrm{gpm}\end{array}$} \\
\hline LIC & $\begin{array}{l}-60 \mathrm{~F} 201 \\
(88981)\end{array}$ & & $\begin{array}{ll}\text { AH : } 85 \% & \text { Alarm - Stop 1st RO Feed Pumps and } \\
\text { SV : } 50 \% & \text { Booster Pump. } \\
\text { AL : 10\% } & \text { Alarm - Stop 2nd RO Feed Tank Pumps } \\
& \text { 2025E-60F-P-2A,B }\end{array}$ & \\
\hline $\mathrm{TI}$ & $\begin{array}{r}-60 \mathrm{~F} 202 \\
(88981) \\
\end{array}$ & $\begin{array}{l}\text { 2nd RO Feed Tank } \\
\text { Temperature }\end{array}$ & $\begin{array}{l}\text { Operating Range: } 90^{\circ} \mathrm{F} \\
\text { AH : } 113^{\circ} \mathrm{F} \text { Alarm - Stop 2nd RO Feed Tank Pump } \\
\text { 2025E-60F-P-2A,B }\end{array}$ & Maximum operating temperature : $113^{\circ} \mathrm{F}$ \\
\hline PI & $\begin{array}{l}-60 \mathrm{~F} 211 \\
(88981)\end{array}$ & $\begin{array}{l}\text { 2nd RO Feed Tank } \\
\text { Pump Discharge } \\
\text { Pressure }\end{array}$ & $\begin{array}{l}\text { Operating: } 490 \text { psig } \\
\text { AH : } 575 \text { psig Alarm - Stop 2nd RO Feed Tank Pump } \\
\begin{array}{l}2025-E-60 F-P-2 A, B \\
\text { AL : } 350 \text { psig Alarm }\end{array} \\
\end{array}$ & $\begin{array}{l}\text { Prevent Second RO Membranes from Damage due } \\
\text { to Over Pressure. } \\
\text { Prevent Pumps from Running Dry. }\end{array}$ \\
\hline II & $\begin{array}{r}-60 \mathrm{~F} 232 \\
(88981) \\
\end{array}$ & $\begin{array}{l}\text { 2nd RO Feed Tank } \\
\text { Pump (A) Current }\end{array}$ & FLA: $97 \mathrm{~A}$ & FLA: Full Load Amperage \\
\hline II & $\begin{array}{r}-60 \mathrm{~F} 234 \\
(88981)\end{array}$ & $\begin{array}{l}\text { 2nd RO Feed Tank } \\
\text { Punp (B) Current }\end{array}$ & FLA: 97 A & FLA: Full Load Amperage \\
\hline
\end{tabular}


TABLE 12 60F REVERSE OSMOSIS (2nd RO Stage)

\begin{tabular}{|c|c|c|c|c|}
\hline \multicolumn{2}{|c|}{$\begin{array}{l}\text { Tag No. (PID No. } \\
\text { H-2-89337) }\end{array}$} & \multirow{2}{*}{\begin{tabular}{|l} 
Name \\
2nd Ro Inlet \\
Pressure
\end{tabular}} & \multirow{2}{*}{$\begin{array}{l}\text { Set-Values and Functions } \\
\text { Operating : } 440 \text { psig } \\
\text { AH : } 575 \text { psi Alarm - Display 2nd RO CLEANING } \\
\text { OPERATION REQUIRED } \\
\text { SV: FIC-60F263 }\end{array}$} & \multirow{2}{*}{$\begin{array}{l}\text { Remarks } \\
\text { To show cleaning operation is required }\end{array}$} \\
\hline PIC & $-60 \mathrm{~F} 250$ & & & \\
\hline PI & $\begin{array}{r}-60 \mathrm{~F} 260 \\
(88982) \\
\end{array}$ & $\begin{array}{l}\text { 2nd RO Brine } \\
\text { Pressure }\end{array}$ & Operating : 410 psig & System Design Process Value. \\
\hline PI & $\begin{array}{r}-60 \mathrm{~F} 261 \\
(88982)\end{array}$ & $\begin{array}{l}\text { 2nd RO Permeate } \\
\text { Pressure }\end{array}$ & $\begin{array}{l}\text { Operating : } 50 \text { psi } \\
\text { AH: } \quad 140 \text { psig Alarm message 2nd RO Permeate Pressure } \\
\text { High }\end{array}$ & $\begin{array}{l}\text { Protect RO Membrane from Damage due to } \\
\text { Excessive Back Pressure. } \\
\text { V-135A-003-052 }\end{array}$ \\
\hline FIC & $\begin{array}{r}-60 \mathrm{~F} 262 \\
(88982) \\
\end{array}$ & 2nd RO Brine Flow & $\begin{array}{l}\text { Operating : } 9 \mathrm{gpm} \\
\text { SV : (FI-60F263) } \times 0.06\end{array}$ & CS : FCV-60F262 \\
\hline FIC & $\begin{array}{r}-60 \mathrm{~F} 263 \\
(88982) \\
\end{array}$ & $\begin{array}{l}\text { 2nd Ro Permeate } \\
\text { Flow }\end{array}$ & $\begin{array}{l}\text { Operating : } 156 \mathrm{gpm} \\
\text { SV : LIC-60F201 }\end{array}$ & CS : PIC-60F250 \\
\hline $\begin{array}{l}\text { AI } \\
(\mathrm{COND})\end{array}$ & $\begin{array}{r}-60 \mathrm{~F} 265 \\
(88982) \\
\end{array}$ & $\begin{array}{l}\text { 2nd RO Brine } \\
\text { Conductivity }\end{array}$ & $\begin{array}{l}\text { Operating : } 12,000 \text { E-6 S/cm } \\
\text { AH: } 15,000 \mathrm{E}-6 \mathrm{~S} / \mathrm{cm} \text { Alarm message }\end{array}$ & Design : 8090 ppm \\
\hline $\begin{array}{l}\text { AI } \\
(\mathrm{PH})\end{array}$ & $\begin{array}{l}-60 \mathrm{~F} 266 \\
(88982) \\
\end{array}$ & 2nd RO Brine $\mathrm{pH}$ & $\begin{array}{ll}\text { Operating }: & 4.5(0-14) \\
\text { AH: } 6.0 & \text { Alarm message } \\
\text { AL: } 3.5 & \text { Alarm message }\end{array}$ & $\begin{array}{l}\text { Protect RO Membranes from Damage due to High } \\
\text { or Low pH. } \\
\text { V-135A-003-052 }\end{array}$ \\
\hline TI & $\begin{array}{r}-60 \mathrm{~F} 269 \\
(88982) \\
\end{array}$ & $\begin{array}{l}\text { 2nd RO Permeate } \\
\text { Temperature }\end{array}$ & $\begin{array}{l}\text { Operating : } 92^{\circ} \mathrm{F} \\
\text { AH: } 113^{\circ} \mathrm{F} \quad \text { Alarm - Stop 2nd RO Feed Pump }\end{array}$ & $\begin{array}{l}\text { Protoct membranes from High Temperature. } \\
\text { V-135A-003-052 }\end{array}$ \\
\hline $\begin{array}{l}\text { AI } \\
\text { (COND) }\end{array}$ & $\begin{array}{r}-60 \mathrm{~F} 270 \\
(88982) \\
\end{array}$ & $\begin{array}{l}\text { 2nd RO Permeate } \\
\text { Conductivity }\end{array}$ & $\begin{array}{l}\text { Operating : } 40 \mathrm{E}-6 \mathrm{~S} / \mathrm{cm} \\
\text { AH: } 100 \mathrm{E}-6 \mathrm{~S} / \mathrm{cm} \quad \text { Alarm message }\end{array}$ & $\begin{array}{l}\text { Warn Operator of Fouled or Ruptured } \\
\text { Membranes. V-135A-003-052 }\end{array}$ \\
\hline $\begin{array}{l}\mathrm{AI} \\
(\mathrm{pH})\end{array}$ & $\begin{array}{r}-60 \mathrm{~F} 271 \\
(88982) \\
\end{array}$ & 2nd RO Permeate pH & $\begin{array}{ll}\text { Operating : } 2.2 & \\
\text { AH: } 3.5 & \text { Alarm message } \\
\text { AL: } 1.5 & \text { Alarm message }\end{array}$ & $\begin{array}{l}\text { Protect RO Membranes from Damage due to High } \\
\text { or Low pH. } \\
\text { V-135A-003-052 }\end{array}$ \\
\hline FIC & $\begin{array}{r}-60 F 273 \\
(88982) \\
\end{array}$ & $\begin{array}{l}\text { 2nd RO Permeate } \\
\text { Recirculation Flow }\end{array}$ & $\begin{array}{l}\text { Operating : } 0 \text { - } 156 \mathrm{gpm} \\
\text { SV : LIC-60F101 or FIT-60D007 }\end{array}$ & CS : FCV-60F273 \\
\hline $\begin{array}{l}\text { AI } \\
\text { (COND) }\end{array}$ & $\begin{array}{l}-60 \mathrm{~F} 274 \\
(88982) \\
\end{array}$ & $\begin{array}{l}\text { 2nd RO Sample } \\
\text { Concentrate } \\
\text { Conductivity } \\
\end{array}$ & $1,200 \sim 5,00 \mathrm{E}-6 \mathrm{~s} / \mathrm{cm}$ & System Design Process Value. \\
\hline $\begin{array}{l}\text { AI } \\
(C O N D)\end{array}$ & $\begin{array}{r}-60 F 275 \\
(88982)\end{array}$ & $\begin{array}{l}\text { 2nd RO Sample } \\
\text { Permeate } \\
\text { Conductivity }\end{array}$ & Operating : $\quad 10 \sim 30 \mathrm{E}-6 \mathrm{~s} / \mathrm{cm}$ & System Design Process Value. \\
\hline
\end{tabular}


TABLE 13 60F REVERSE OSMOSIS (RO/CIP System, Scale Inhibitor Dosing System) ZENON

\begin{tabular}{|c|c|c|c|c|}
\hline \multicolumn{2}{|c|}{$\begin{array}{l}\text { Tag No. (PID No. } \\
\text { H-2-89337) }\end{array}$} & \multirow{2}{*}{$\begin{array}{l}\text { Name } \\
\text { Cleaning Liquid } \\
\text { Return Flow } \\
\end{array}$} & \multirow[t]{2}{*}{ Set-Values and Functions } & \multirow{2}{*}{ Remarks } \\
\hline FI & $\begin{array}{r}-60 \mathrm{~F} 301 \\
(89343) \\
\end{array}$ & & & \\
\hline LI & $\begin{array}{r}-60 F 303 \\
(89343) \\
\end{array}$ & CIP Tank Level & $\begin{array}{ll}\text { AH: } 90 \% & \text { Alarm } \\
\text { AL: } 10 \% & \text { Alarm - Stop CIP Pump 2025E-60F-P-04 }\end{array}$ & $\begin{array}{l}\text { Tank Level Control } \\
\text { V-135A-003-052 }\end{array}$ \\
\hline PI & (89343) & $\begin{array}{l}\text { CIP Pump Discharge } \\
\text { Pressure }\end{array}$ & $\begin{array}{ll}\text { Operating: } 52 \text { psi } \\
\text { AH: } & \text { 55 psi Alarm - Stop CIP Pump } \\
& 2025 \mathrm{E}-60 \mathrm{~F}-\mathrm{P}-04 \\
& \\
\text { AL: } & 25 \text { psi Alarm - Stop CIP Pump } \\
& 2025 \mathrm{E}-60 \mathrm{~F}-\mathrm{P}-04 \\
\end{array}$ & $\begin{array}{l}\text { CIP Pump Operative Range. } \\
\text { V-135A-003-052 }\end{array}$ \\
\hline II & $\begin{array}{r}-60 F 306 \\
(89343) \\
\end{array}$ & CIP Pump Current & FLA: $3.4 \mathrm{~A}$ & FLA: Full Load Amperage \\
\hline TI & $\begin{array}{r}-60 F 307 \\
(89343) \\
\end{array}$ & $\begin{array}{l}\text { CIP Pump Outlet } \\
\text { Temperature }\end{array}$ & $\begin{array}{cc}\text { AH: } & 104^{\circ} \mathrm{F} \text { Alarm } \\
& \text { Stop CIP Pump }\end{array}$ & $\begin{array}{l}\text { Prevent damage to RO Membrane due to High } \\
\text { Temperature. }\end{array}$ \\
\hline LS & $\begin{array}{r}-60 F 308 \\
(88979)\end{array}$ & $\begin{array}{l}\text { Scale Inhibitor } \\
\text { Dosing Tank Level }\end{array}$ & $\begin{array}{l}\text { AL: } 6 \text { inch Alarm Message - SCALE INHIBITOR } \\
\text { SUPPLY REQUIRED (480 minutes operation after } \\
\text { Low alarm). Stop scale inhibitor dosing pump } \\
\text { 2025E-60F-P-5 }\end{array}$ & $\begin{array}{l}\text { Tank Size: } 21 \text { " } \Phi \times 26^{\prime \prime} \text { high } \\
\text { Prevent operation without Scale Inhibitor. } \\
\text { V-135A-003-052 }\end{array}$ \\
\hline
\end{tabular}


TABLE 14 60G ION EXCHANGE SYSTEM (Polisher System)

\begin{tabular}{|c|c|c|c|c|}
\hline \multicolumn{2}{|c|}{$\begin{array}{l}\text { Tag No. (PID No. } \\
\text { H-2-89337) }\end{array}$} & \multirow{2}{*}{\begin{tabular}{|l|} 
Name \\
$\begin{array}{l}\text { Polisher Verification } \\
\text { Water Flow }\end{array}$ \\
\end{tabular}} & \multirow{2}{*}{$\begin{array}{l}\text { Set-Values and Functions } \\
\text { Operating : } 20-100 \mathrm{gpm}\end{array}$} & \multirow{2}{*}{ Remarks } \\
\hline FI & $\begin{array}{r}-60 G 001 \\
(88983)\end{array}$ & & & \\
\hline FI & $\begin{array}{r}-60 \mathrm{G002} \\
(88983)\end{array}$ & Polisher SA Flow & Operating : $110 \mathrm{scfm}$ & System Design Process Value. \\
\hline $\begin{array}{l}\text { PI } \\
\text { (Local) }\end{array}$ & $\begin{array}{r}-60 G 005 \\
(88983) \\
\end{array}$ & $\begin{array}{l}\text { Service Air Inlet } \\
\text { Pressure }\end{array}$ & Operating : 90 psig & System Design Process Value. \\
\hline $\begin{array}{l}\text { PI } \\
\text { (Local) }\end{array}$ & $\begin{array}{r}-60 G 006 \\
(88983)\end{array}$ & $\begin{array}{l}\text { Service Air } \\
\text { Regulator Outlet } \\
\text { Pressure } \\
\end{array}$ & Operating : (80) psig & Air to Operate Values. \\
\hline PI & $\begin{array}{r}-60 G 100 \\
(88983) \\
\end{array}$ & $\begin{array}{l}\text { Polisher System Inlet } \\
\text { Pressure } \\
\end{array}$ & $\begin{array}{l}\text { Operating : } 70 \text { psig } \\
\text { AH: } 100 \text { psig Stop 2nd RO Feed Pump }\end{array}$ & Prevent Damage due to High Pressure. \\
\hline PI & $\begin{array}{r}-60 G 101 \\
(88983) \\
\end{array}$ & $\begin{array}{l}\text { Resin Strainer A } \\
\text { Outlet Pressure } \\
\end{array}$ & $\begin{array}{r}\text { Operating : } 45 \text { psig as Ist column } \\
20 \text { psig as 2nd column }\end{array}$ & System Design Process Value. \\
\hline PI & $\begin{array}{r}-60 G 102 \\
(88983) \\
\end{array}$ & \begin{tabular}{|l} 
Polisher A Outlet \\
Pressure \\
\end{tabular} & $\begin{array}{r}\text { Operating : } 50 \text { psig as 1st column } \\
25 \text { psig as } 2 n d \text { column }\end{array}$ & System Design Process Value. \\
\hline $\begin{array}{l}\text { AI } \\
\text { (COND) }\end{array}$ & $\begin{array}{r}-60 G 104 \\
(88983) \\
\end{array}$ & $\begin{array}{l}\text { Polisher A Outlet } \\
\text { Conductivity }\end{array}$ & $\begin{array}{l}\text { Operating : } 0.1 \mathrm{E}-6 \mathrm{~S} / \mathrm{cm} \\
\text { AH: } 1 \text { E-6 S/cm Alarm } \\
\text { - Change AOV-60G401 to "Surge Tank" } \\
\text { - Regeneration when used as 1st column }\end{array}$ & $\begin{array}{l}\text { When Polisher (A)is used as } 2 \text { nd column. } \\
\text { System Design Process Value. }\end{array}$ \\
\hline $\begin{array}{l}\mathrm{AI} \\
(\mathrm{pH})\end{array}$ & $\begin{array}{r}-60 G 105 \\
(88983)\end{array}$ & Polisher A Outlet pH & Operating : $5.5-8.5$ & $\begin{array}{l}\text { System Design } \\
\text { V-135A-006-075 }\end{array}$ \\
\hline PDI & $\begin{array}{r}-60 G 107 \\
(88983) \\
\end{array}$ & $\begin{array}{l}\text { Polisher A } \\
\text { Differential Pressure }\end{array}$ & Operating : 20 psig & $\begin{array}{l}\text { Calculated by OCS } \\
\text { if } 1 \text { st column : PI-60G100-PI-60G102 } \\
\text { if 2nd column : PI-60G301-PI-60G102 }\end{array}$ \\
\hline PDI & $\begin{array}{r}-60 G 108 \\
(88983)\end{array}$ & $\begin{array}{l}\text { Resin Strainer A } \\
\text { Differential Pressure }\end{array}$ & Operating : 5 psig & $\begin{array}{l}\text { Calculated by OCS : } \\
\text { PI-60G102-PI-60G101 }\end{array}$ \\
\hline PI & $\begin{array}{r}-60 G 201 \\
(88983) \\
\end{array}$ & $\begin{array}{l}\text { Resin Strainer B } \\
\text { Outlet Pressure } \\
\end{array}$ & $\begin{array}{r}\text { Operating : } 45 \text { psig as 1st column } \\
20 \text { psig as } 2 \text { nd column }\end{array}$ & System Design Process Value. \\
\hline PI & $\begin{array}{r}-60 G 202 \\
(88983) \\
\end{array}$ & $\begin{array}{l}\text { Polisher B Outlet } \\
\text { Pressure }\end{array}$ & $\begin{array}{c}\text { Operating : } 50 \text { psig as } 1 \text { st column } \\
25 \text { psig as } 2 \text { nd column }\end{array}$ & System Design Process Value. \\
\hline $\begin{array}{l}\text { AI } \\
\text { (COND) }\end{array}$ & $\begin{array}{r}-606204 \\
(88983)\end{array}$ & $\begin{array}{l}\text { Polisher B Outlet } \\
\text { Conductivity }\end{array}$ & $\begin{array}{l}\text { Operating : } 0.1 \mathrm{E}-6 \mathrm{~S} / \mathrm{cm} \\
\text { AH: } 1 \mathrm{E}-6 \mathrm{~S} / \mathrm{cm} \text { Alarm } \\
\text { Change AOV-60G401 to "Surge Tank" Regeneration } \\
\text { when used as } 1 \text { st column }\end{array}$ & When polisher (B) is used as 2 nd column. \\
\hline
\end{tabular}


TABLE 14 60G ION EXCHANGE SYSTEM (Polisher System)

\begin{tabular}{|c|c|c|c|c|}
\hline \multicolumn{2}{|c|}{$\begin{array}{l}\text { Tag No. (PID No. } \\
\text { H-2-89337) }\end{array}$} & \multirow{2}{*}{ Name } & \multirow{2}{*}{ Set-Values and Functions } & \multirow{2}{*}{$\begin{array}{l}\text { Remarks } \\
\text { System Design. } \\
\text { V-135A-006-075 }\end{array}$} \\
\hline $\begin{array}{l}\mathrm{AI} \\
(\mathrm{pH})\end{array}$ & $\begin{array}{r}-60 G 205 \\
(88983) \\
\end{array}$ & & & \\
\hline PDI & $\begin{array}{r}-60 G 207 \\
(88983)\end{array}$ & $\begin{array}{l}\text { Polisher B } \\
\text { Differential Pressure }\end{array}$ & Operating: 20 psig & $\begin{array}{l}\text { Calculated by OCS } \\
\text { if 1st columm : PI-60G100-PI-60G202 } \\
\text { if } 2 \text { nd column : PI-60G101-PI-60G202 }\end{array}$ \\
\hline PDI & $\begin{array}{r}-60 G 208 \\
(88983) \\
\end{array}$ & $\begin{array}{l}\text { Resin Strainer B } \\
\text { Differential Pressure }\end{array}$ & Operating : 5 psig & $\begin{array}{l}\text { Calculated by OCS : } \\
\text { PI-60G202 - PI-60G201 }\end{array}$ \\
\hline PI & $\begin{array}{r}-60 G 301 \\
(88983) \\
\end{array}$ & $\begin{array}{l}\text { Resin Strainer C } \\
\text { Outlet Pressure }\end{array}$ & $\begin{array}{c}\text { Operating : } 45 \text { psig as 1st column } \\
20 \text { psig as } 2 \text { nd column }\end{array}$ & System Design Process Value. \\
\hline PI & $\begin{array}{r}-60 G 302 \\
(88983) \\
\end{array}$ & $\begin{array}{l}\text { Polisher C Outlet } \\
\text { Pressure }\end{array}$ & $\begin{array}{l}\text { Operating : } 50 \text { psig as 1st column } \\
25 \text { psig as 2nd column } \\
\end{array}$ & System Design Process Value \\
\hline $\begin{array}{l}\text { AI } \\
\text { (COND) }\end{array}$ & $\begin{array}{r}-60 G 304 \\
(88983)\end{array}$ & $\begin{array}{l}\text { Polisher C Outlet } \\
\text { Conductivity }\end{array}$ & $\begin{array}{l}\text { Operating : } 0.1 \text { E-6 S/cm } \\
\text { AH: } 1 \text { E-6 S/cm Alarm } \\
\text { Change AOV-60G401 to "Surge Tank" } \\
\text { Regeneration when used as 1st column }\end{array}$ & When polisher (C) is used as 2 nd column \\
\hline $\begin{array}{l}\mathrm{AI} \\
(\mathrm{pH})\end{array}$ & $\begin{array}{r}-60 G 305 \\
(88983) \\
\end{array}$ & Polisher C Outlet pH & Operating : $5.5-8.5$ & $\begin{array}{l}\text { System Design. } \\
\text { V-135A-006-075 }\end{array}$ \\
\hline PDI & $\begin{array}{r}-60 G 307 \\
(88983)\end{array}$ & $\begin{array}{l}\text { Polisher C } \\
\text { Differential Pressure }\end{array}$ & Operating : 20 psig & $\begin{array}{l}\text { Calculated by OCS } \\
\text { if 1st column : PI-60G100-PI-60G302 } \\
\text { if 2nd column : PI-60G201-PI-60G302 }\end{array}$ \\
\hline PDI & $\begin{array}{r}-60 G 308 \\
(88983)\end{array}$ & $\begin{array}{l}\text { Resin Strainer } \\
\text { C Differential } \\
\text { Pressure } \\
\end{array}$ & Operating : 5 psig & $\begin{array}{l}\text { Calculated by OCS : } \\
\text { PI-60G302 - PI-60G301 }\end{array}$ \\
\hline LS & $\begin{array}{r}-60 G 501 \\
(88983) \\
\end{array}$ & $\begin{array}{l}\text { Polisher (A) } \\
\text { Level }\end{array}$ & Finish Blowdown 2 and 3 & $\begin{array}{l}\text { Design } \\
\text { V-135A-006-075 }\end{array}$ \\
\hline LS & $\begin{array}{r}-60 G 502 \\
(88983) \\
\end{array}$ & $\begin{array}{l}\text { Polisher (B) } \\
\text { Level }\end{array}$ & Finish Blowdown 2 and 3 & $\begin{array}{l}\text { Design } \\
\text { V-135A-006-075 } \\
\end{array}$ \\
\hline LS & $\begin{array}{r}-606503 \\
(88983)\end{array}$ & $\begin{array}{l}\text { Polisher (C) } \\
\text { Level }\end{array}$ & Finish Blowdown 2 and 3 & $\begin{array}{l}\text { Design } \\
\text { V-135A-006-075 }\end{array}$ \\
\hline
\end{tabular}


TABLE 15 60H EFFLUENT VERIFICATION (Verification System)

\begin{tabular}{|c|c|c|c|c|}
\hline $\begin{array}{l}\text { Tag No. (PID No. } \\
\text { H-2-89337) }\end{array}$ & \multirow{2}{*}{$\begin{array}{l}\text { Name } \\
\begin{array}{l}\text { Verification Tank(A) } \\
\text { Level }\end{array}\end{array}$} & \multicolumn{2}{|c|}{ Set-Values and Functions } & \multirow{2}{*}{$\begin{array}{l}\text { Remarks } \\
\text { See Equipment Process Calculation } \\
\text { System Design Process Values. }\end{array}$} \\
\hline (88985) & & $\begin{array}{l}\text { AHH : } 95 \% \\
\text { AH : } 93 \% \\
\text { AL : } 7 \% \\
\text { ALL : } 5 \%\end{array}$ & $\begin{array}{l}\text { Alarm } \\
\text { Stop Effluent pH Adjustment Tank Pumps } \\
\text { 2025E-60C-P-2A,B } \\
\text { (At "RECEIVING" Mode) } \\
\text { Alarm message } \\
\text { (At "RECEIVING" Mode) } \\
\text { Alarm message } \\
\text { Stop Transfer Pump } \\
\text { (at "DISCHARGE" mode) } \\
\text { Alarm } \\
\text { Stop Retum Pump 2025E-60H-P-1 } \\
\text { (at "VERIFYING" mode) }\end{array}$ & \\
\hline$-60 \mathrm{H001B}$ & $\begin{array}{l}\text { Verification Tank(B) } \\
\text { Level }\end{array}$ & $\begin{array}{l}\text { AHH : } 95 \% \\
\text { AH : } 93 \% \\
\text { AL : } 7 \% \\
\text { ALL : } 5 \%\end{array}$ & $\begin{array}{l}\text { Alarm } \\
\text { Stop Effluent pH Adjustment Tank Pumps } \\
2025 E-60 C-P-2 A, B \\
\text { (at "RECEIVING" Mode) } \\
\text { Alarm message } \\
\text { (at "RECEIVING" Mode) } \\
\text { Alarm message } \\
\text { Stop Transfer Pump } \\
\text { (at "DISCHARGE" Mode) } \\
\text { Alarm } \\
\text { Stop Retum Pump 2025E-60H-P-1 } \\
\text { (at "VERIFYING" mode) }\end{array}$ & Same as LI-60HoOtA \\
\hline $\begin{array}{r}-60 \mathrm{H} 001 \mathrm{C} \\
\\
\\
\\
\end{array}$ & $\begin{array}{l}\text { Verification Tank(C) } \\
\text { Level }\end{array}$ & $\begin{array}{l}\text { AHH : } 95 \% \\
\text { AH : } 93 \% \\
\text { AL : } 7 \% \\
\text { ALL : } 5 \%\end{array}$ & $\begin{array}{l}\text { Alarm } \\
\text { Stop Effluent pH Adjustment Tank Pumps } \\
2025 E-60 C-P-2 A, B \\
\text { (at "RECEIVING" Mode) } \\
\text { Alarm message } \\
\text { (at "RECEIVING" Mode) } \\
\text { Alarm message } \\
\text { Stop Transfer Pump } \\
\text { (at "DISCHARGE" Mode) } \\
\text { Alarm } \\
\text { Stop Returm Pump 2025E-60H-P-1 } \\
\text { (at "VERIFYING" mode) }\end{array}$ & Same as LI-60H001A \\
\hline $\begin{array}{r}\text { TI } \quad-60 \mathrm{H} 002 \mathrm{~A} \\
(88985)\end{array}$ & $\begin{array}{l}\text { Verification } \operatorname{Tank}(\mathrm{A}) \\
\text { Temperature }\end{array}$ & $\begin{array}{l}\text { AH : } 110^{\circ} \mathrm{F} \\
\text { SH : } 60^{\circ} \mathrm{F} \\
\text { SL }: 50^{\circ} \mathrm{F} \\
\text { AL : } 41^{\circ} \mathrm{F}\end{array}$ & $\begin{array}{l}\text { Alarm } \\
\text { Tum off freeze-protection heater } \\
\text { Tum on freeze-protection heater } \\
\text { Alarm }\end{array}$ & $\begin{array}{l}\text { Lower than max. operating temperature } 120^{\circ} \mathrm{F} \\
\text { SL }+10^{\circ} \mathrm{F} \\
\text { Maximum density point of water }\end{array}$ \\
\hline
\end{tabular}


TABLE 15 60H EFFLUENT VERIFICATION (Verification System)

\begin{tabular}{|c|c|c|c|}
\hline \begin{tabular}{|l} 
Tag No. (PID No. \\
H-2-89337) \\
\end{tabular} & \multirow{2}{*}{$\begin{array}{l}\text { Name } \\
\text { Verification Tank(B) } \\
\text { Temperature } \\
\end{array}$} & \multirow{2}{*}{$\begin{array}{l}\text { Set-Values and Functions } \\
\text { Same as TI-60H002A }\end{array}$} & \multirow{2}{*}{$\frac{\text { Remarks }}{\text { Same as TI-60H002A }}$} \\
\hline $\begin{array}{r}-60 \mathrm{H} 002 \mathrm{~B} \\
(88985) \\
\end{array}$ & & & \\
\hline $\begin{array}{r}-60 \mathrm{H} 002 \mathrm{C} \\
(88985) \\
\end{array}$ & $\begin{array}{l}\text { Verification Tank(C) } \\
\text { Temperature }\end{array}$ & Same as $\mathrm{TI}-60 \mathrm{H} 002 \mathrm{~A}$ & Same as TI-60H002A \\
\hline $\begin{array}{r}-60 \mathrm{H} 003 \mathrm{~A} \\
(88985) \\
\end{array}$ & $\begin{array}{l}\text { Verification Tank } \\
\text { (A) Level } \\
\end{array}$ & AH: $\quad 97 \%$ Alarm & $\begin{array}{l}\text { Back-Up for LI-60H001A } \\
\text { H(LS-60H001A) + 2\% }\end{array}$ \\
\hline $\begin{array}{r}-60 \mathrm{H} 003 \mathrm{~B} \\
(88985) \\
\end{array}$ & $\begin{array}{l}\text { Verification Tank (B) } \\
\text { Level }\end{array}$ & Same as LS-60H003A & Same as LS-60H003A \\
\hline $\begin{array}{r}-60 \mathrm{H} 003 \mathrm{C} \\
(88985) \\
\end{array}$ & $\begin{array}{l}\text { Verification Tank } \\
\text { (C) Level }\end{array}$ & Same as $\mathrm{LS}-60 \mathrm{H} 003 \mathrm{~A}$ & Same as $\mathrm{LS}-60 \mathrm{H} 003 \mathrm{~A}$ \\
\hline $\begin{array}{r}-60 \mathrm{H013} \\
(88985) \\
\end{array}$ & $\begin{array}{l}\text { Return Pump } \\
\text { Discharge Pressure }\end{array}$ & SV: 90 psig & System Process Design Value. \\
\hline $\begin{array}{r}-60 \mathrm{H016} \\
(88985) \\
\end{array}$ & $\begin{array}{l}\text { Verification Tank } \\
\text { Effluent Return Flow }\end{array}$ & Operating Range: $0-150 \mathrm{gpm}$ & System Process Design Value. \\
\hline $\begin{array}{r}-60 \mathrm{H} 032 \\
(88985) \\
\end{array}$ & Return Pump Current & FLA: $38 \mathrm{~A}$ & $V-1358-002-324$ \\
\hline $\begin{array}{r}-60 \mathrm{H} 034 \\
(88985) \\
\end{array}$ & $\begin{array}{l}\text { Transfer Pump (A) } \\
\text { Current }\end{array}$ & FLA: $56.6 \mathrm{~A}$ & FLA: Full Load Amperage \\
\hline $\begin{array}{r}-60 \mathrm{H} 036 \\
(88985) \\
\end{array}$ & $\begin{array}{l}\text { Transfer Pump (B) } \\
\text { Current }\end{array}$ & FLA: $56.6 \mathrm{~A}$ & FLA: Full Load Amperage \\
\hline $\begin{array}{r}-60 \mathrm{H} 037 \\
(88985) \\
\end{array}$ & $\begin{array}{l}\text { Transfer Pump } \\
\text { Discharge Pressure }\end{array}$ & Operating : 130 psig & Design: Pump outlet point 147 psig \\
\hline $\begin{array}{r}-60 \mathrm{H} 114 \\
(88985) \\
\end{array}$ & $\begin{array}{l}\text { Verification Tank } \\
\text { Discharge Flow }\end{array}$ & SV : (300 gpm) & System Design Process Value. \\
\hline FI $\begin{array}{r}-60 \mathrm{H} 129 \\
\text { (Local) }(88991) \\
\end{array}$ & $\begin{array}{l}\text { Sump Pump 1A, 1B } \\
\text { Seal Water Flow }\end{array}$ & Operating : $2 \sim 4 \mathrm{gpm}$ & $V-1358-002-002$ \\
\hline $\begin{array}{lr}\text { FI } & -60 \mathrm{H} 136 \\
\text { (Local) } & (88991)\end{array}$ & $\begin{array}{l}\text { Sump Pump 2A, 2B } \\
\text { Seal Water Flow }\end{array}$ & Operating : $2 \sim 4 \mathrm{gpm}$ & $V-1358-002-002$ \\
\hline
\end{tabular}


TABLE 16 60I WASTE EVAPORATION (Secondary Waste Receiving System)

\begin{tabular}{|c|c|c|c|}
\hline $\begin{array}{l}\text { Tag No. (PID No. } \\
\text { H-2-89337) }\end{array}$ & Name & Set-Values and Functions & Remarks \\
\hline (88986) & $\begin{array}{l}\text { Secondary Waste } \\
\text { Receiving Tank(A) } \\
\text { Level }\end{array}$ & $\begin{array}{l}\text { AHH : } 95 \% \text { Alarm } \\
\text { Shift positions of AOV-60B019, 60F013, 60G001, } \\
\text { and Close AOV-65C058, 65C059 } \\
\text { If H alarm of LS-601001B has already annunciated, } \\
\text { shift MTT from "OPERATION" to "READY" } \\
\text { AH : } 93 \% \text { Alarm Message Display } \\
\text { SL : } 47 \% \text { Starting condition of Polisher regeneration } \\
\text { AL : } 7 \% \text { Alarm message } \\
\text { Close AOV-601017 } \\
\text { Change to "HOT STANDBY" } \\
\text { ALL : } 5 \% \text { Alarm - Stop Secondary Waste Feed Pump (A) } \\
\text { 2025E-60I-PLA }\end{array}$ & . \\
\hline $\begin{array}{r}-601001 B \\
(88986) \\
\end{array}$ & $\begin{array}{l}\text { Secondary Waste } \\
\text { Receiving Tank(B) } \\
\text { Level }\end{array}$ & $\begin{array}{l}\text { AHH: } 95 \% \text { Alarm } \\
\text { Shift positions of AOV-60B019, 60F013, 60G001, } \\
\text { and close AOV-65C045, 046 } \\
\text { If H alarm of LS-601001A has already annunciated, } \\
\text { Shift Main Treatment Train from "OPERATION" } \\
\text { mode to "READY" } \\
\text { AH : } 93 \% \text { Alarm message Display } \\
\text { SL : } 47 \% \text { Starting condition of Polisher regeneration } \\
\text { AL : } 7 \% \text { Alarm message - Close AOV-60I-016 Change to } \\
\text { ALL : } 5 \% \text { Alarm - Stop Secondary Waste Feed pump (B) } \\
2025 \text {-60I-P-1B }\end{array}$ & Same as LI-60I001A \\
\hline $\begin{array}{r}-601002 A \\
(88986)\end{array}$ & $\begin{array}{l}\text { Secondary Waste } \\
\text { Receiving Tank(A) } \\
\text { Temperature }\end{array}$ & Operating Range: $65-140^{\circ} \mathrm{F}$ & $\begin{array}{l}\text { Influent RO concentrate } 17 \mathrm{gpm} \\
88^{\circ} \mathrm{F} \\
\begin{array}{ll}\text { Filter backwash } 1.5 \mathrm{gpm} & <140^{\circ} \mathrm{F} \\
\text { Polisher regeneration } 0.6 \mathrm{gpm} & <110^{\circ} \mathrm{F}\end{array}\end{array}$ \\
\hline $\begin{array}{r}-601002 \mathrm{~B} \\
(88986) \\
\end{array}$ & $\begin{array}{l}\text { Secondary Waste } \\
\text { Receiving Tank(B) } \\
\text { Temperature }\end{array}$ & Same as LI-60I002A & Same as LI-60I002A \\
\hline $\begin{array}{r}-601011 A \\
(88986) \\
\end{array}$ & $\begin{array}{l}\text { Secondary Waste } \\
\text { Receiving Tank(A) } \\
\text { Discharge Pressure }\end{array}$ & Operating: 60 psig & System Design Process Value. \\
\hline $\begin{array}{r}-601011 B \\
(88986)\end{array}$ & $\begin{array}{l}\text { Secondary Waste } \\
\text { Receiving Tank(B) } \\
\text { Discharge Pressure }\end{array}$ & Same as PI-60I01 1A & Same as PI-60I011A \\
\hline
\end{tabular}


TABLE 16 60I WASTE EVAPORATION (Secondary Waste Receiving System)

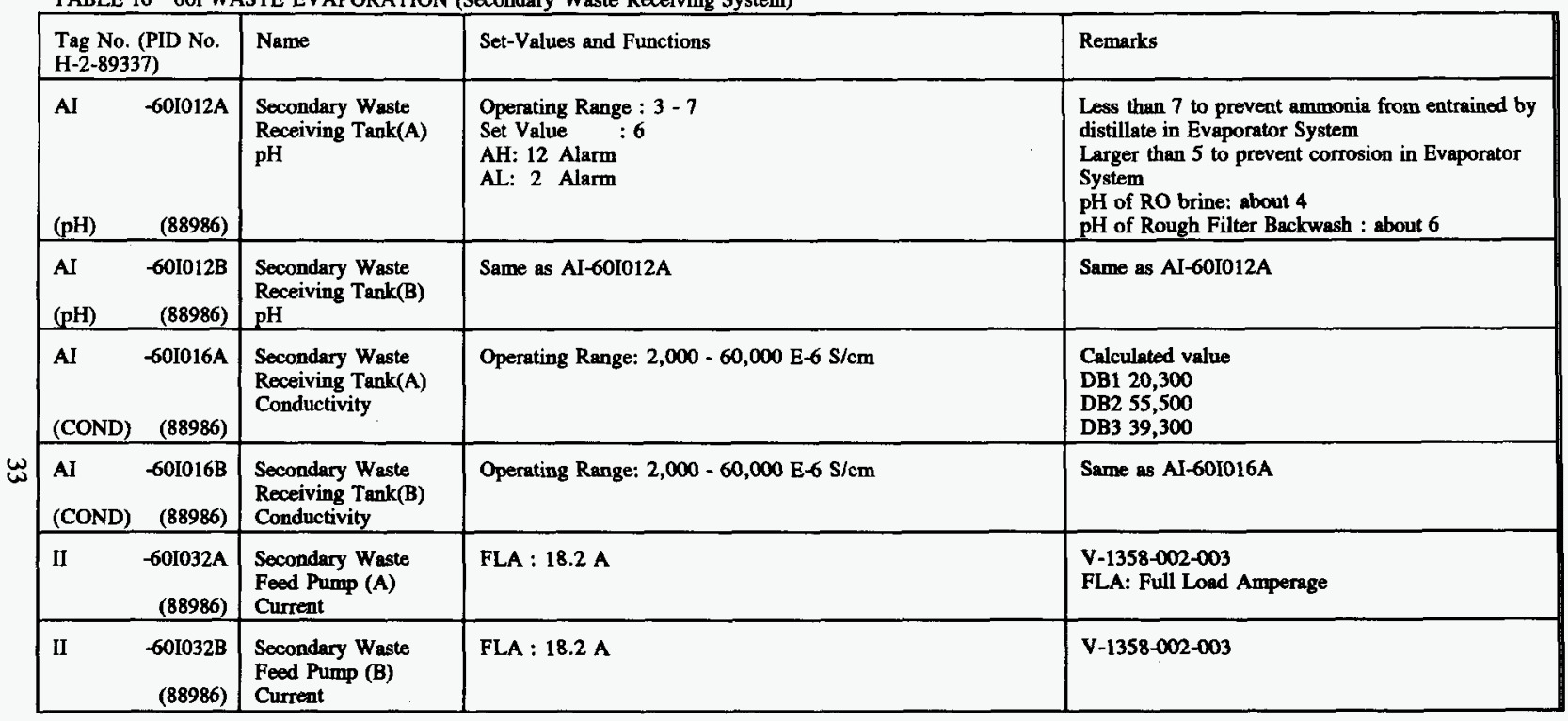


TABLE 17 60I WASTE EVAPORATION (Evaporator System)

\begin{tabular}{|c|c|c|c|c|c|}
\hline \multicolumn{2}{|c|}{$\begin{array}{l}\text { Tag No. (PID No. } \\
\text { H-2-89337) }\end{array}$} & \multirow{2}{*}{$\begin{array}{l}\text { Name } \\
\text { Evaporator Area } \\
\text { Radiation } \\
\end{array}$} & \multicolumn{2}{|c|}{ Set-Values and Functions } & \multirow{2}{*}{ Remarks } \\
\hline RI & $\begin{array}{r}-601035 \\
(89335) \\
\end{array}$ & & \multicolumn{2}{|c|}{ Operating : .01-100 mR/h range, set-value by client } & \\
\hline FI & $\begin{array}{r}-60 \mathrm{I} 102 \\
(88987) \\
\end{array}$ & $\begin{array}{l}\text { Secondary Waste } \\
\text { Evaporator Feed } \\
\text { Flow }\end{array}$ & \multicolumn{2}{|c|}{ Operating : $0 \sim 20 \mathrm{gpm}$} & $\begin{array}{l}\text { Design Flow } \\
\text { V-135A-004 }\end{array}$ \\
\hline FI & $\begin{array}{r}-601103 \\
(88987) \\
\end{array}$ & $\begin{array}{l}\text { Distillate Flash Tank } \\
\text { Discharge Flow }\end{array}$ & \multicolumn{2}{|c|}{ Operating : $0 \sim 23 \mathrm{gpm}$} & $\begin{array}{l}\text { Design Flow } \\
\text { V-135A-004 }\end{array}$ \\
\hline Al & $\begin{array}{r}-60 \mathrm{I} 104 \\
(88987) \\
\end{array}$ & $\begin{array}{l}\text { Distillate } \\
\text { Conductivity }\end{array}$ & \multicolumn{2}{|c|}{$\begin{array}{ll}\text { Operating : } 10 & \mathrm{E}-6 \mathrm{~S} / \mathrm{cm} \\
\mathrm{SH}: 1000 \mathrm{E}-6 & \begin{array}{c}\text { Open AOV-60I106 } \\
\text { Close AOV-60I105 }\end{array} \\
\mathrm{AH}: 500 \mathrm{E}-6 & \text { Alarm message } \\
\end{array}$} & Based on design basis \\
\hline DI & $\begin{array}{r}-601106 \\
(89335) \\
\end{array}$ & $\begin{array}{l}\text { Evaporator Vapor } \\
\text { Body Density }\end{array}$ & $\begin{array}{l}\text { AHH: } 1.45 \\
\mathrm{AH}: 1.42 \\
\mathrm{SH}: 1.21\end{array}$ & $\begin{array}{l}\text { Alarm change to "HOT STANDBY" } \\
\text { Alarm } \\
\text { Density exceeds SH } 5 \text { minutes continuously, } \\
\text { then open AOV-60I108 for } 10 \text { seconds }\end{array}$ & $\begin{array}{l}\text { Range: } 0.95-1.5 \\
\text { Calculate for each Waste Stream Processed. }\end{array}$ \\
\hline LIC & -601107 & $\begin{array}{l}\text { Evaporator Vapor } \\
\text { Body Level }\end{array}$ & $\begin{array}{l}\text { AHH : } 90 \% \\
\text { AH }: 85 \% \\
\text { SV }: 50 \% \\
\text { AL }: 35 \% \\
\text { ALL : } 33 \% \\
\text { ALLL: } 10 \%\end{array}$ & $\begin{array}{l}\text { Alarm - Stop Vapor Compressor } \\
\text { (HOT STANDBY) } \\
\text { Alarm } \\
\text { Alarm } \\
\text { Alarm - Stop Vapor Compressor } \\
\text { (HOT STANDBY) } \\
\text { Alarm - Stop Recirculation Pump } \\
\text { 2025E-60I-P-02 }\end{array}$ & $\begin{array}{l}\text { Should be adjusted by using density. } \\
\text { Range: } 7 \\
\text { (V-135A-004-123) } \\
\text { System Design Values for Operation. } \\
\text { Controller set value may vary and will be } \\
\text { controlled by approved operating procedures. }\end{array}$ \\
\hline LIC & -601108 & $\begin{array}{l}\text { Distillate Flash Tank } \\
\text { Level }\end{array}$ & $\begin{array}{l}\text { AHH : } 90 \% \\
\text { AH : } 80 \% \\
\text { SV : } 60 \% \\
\text { AL : } 55 \% \\
\text { ALL : } 15 \%\end{array}$ & $\begin{array}{l}\text { Alarm - Stop Distillate Flash Tank Pump } \\
\text { 2025E-60I-P-03 } \\
\text { Alarm - Start Distillate Flash Tank Pump at } \\
\text { START-UP, HOT-STANDBY mode } \\
\text { Alarm - Stop Distillate Flash Tank Purmp at } \\
\text { START-UP, HOT-STANDBY mode } \\
\text { Alarm - Stop Distillate Flash Tank Pump } \\
\text { 2025E-60I-P-03 }\end{array}$ & $\begin{array}{l}\text { System Design Values for Operation. } \\
\text { V-135A-004-123 }\end{array}$ \\
\hline
\end{tabular}


TABLE 17 60I WASTE EVAPORATION (Evaporator System)

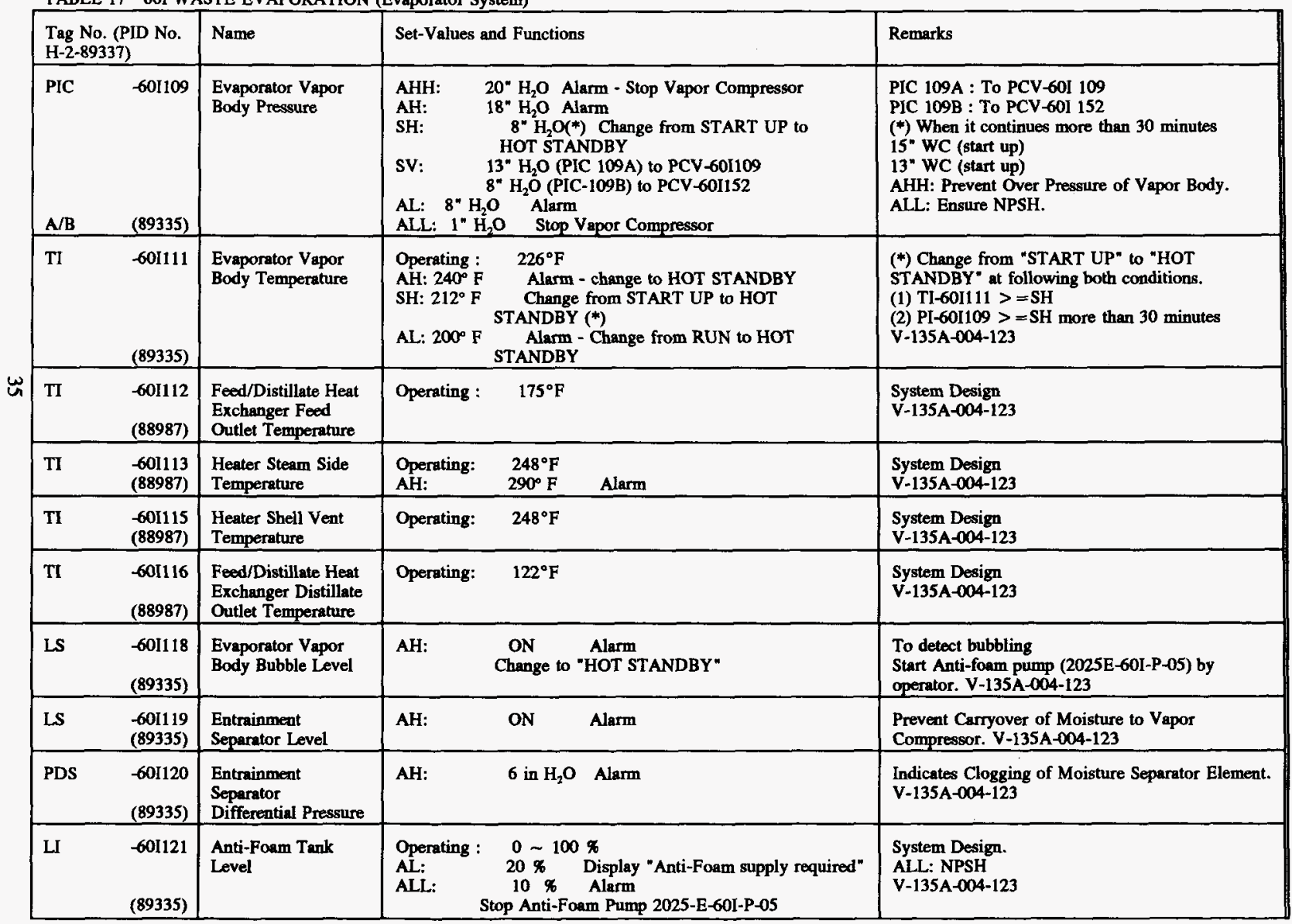


TABLE 17 60I WASTE EVAPORATION (Evaporator System)

\begin{tabular}{|c|c|c|c|c|}
\hline \multicolumn{2}{|c|}{$\begin{array}{l}\text { Tag No. (PID No. } \\
\mathrm{H}-2-89337 \text { ) }\end{array}$} & \multirow{2}{*}{$\begin{array}{l}\text { Name } \\
\text { Distillate Flash Tank } \\
\text { Pump Discharge } \\
\text { Pressure } \\
\end{array}$} & \multirow{2}{*}{$\begin{array}{l}\text { Set-Values and Functions } \\
\text { Operating : } 81 \text { psig }\end{array}$} & \multirow{2}{*}{$\begin{array}{l}\text { Remarks } \\
\text { System Design. } \\
\text { V-135A-004-123 }\end{array}$} \\
\hline PI & $\begin{array}{r}-601122 \\
(88987) \\
\end{array}$ & & & \\
\hline PI & $\begin{array}{r}-60 \mathrm{I} 123 \\
(89335) \\
\end{array}$ & $\begin{array}{l}\text { Recirculation Pump } \\
\text { Discharge Pressure }\end{array}$ & 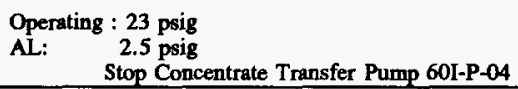 & $\begin{array}{l}\text { It is used when Evaporator drain-out operation } \\
\text { Stops Condensate Transfer Pump. }\end{array}$ \\
\hline Tw & $\begin{array}{r}-601125 \\
(89335) \\
\end{array}$ & $\begin{array}{l}\text { Heater Inlet } \\
\text { Temperature }\end{array}$ & Operating : $228^{\circ} \mathrm{F}$ & $\begin{array}{l}\text { System Design. } \\
\text { V-135A-004-123 }\end{array}$ \\
\hline TW & $\begin{array}{r}-601126 \\
(89335) \\
\end{array}$ & $\begin{array}{l}\text { Heater outlet } \\
\text { Temperature }\end{array}$ & Operating : $233^{\circ} \mathrm{F}$ & $\begin{array}{l}\text { System Design. } \\
\text { V.135A-004-123 }\end{array}$ \\
\hline TI & $\begin{array}{l}-601127 \\
(88987)\end{array}$ & $\begin{array}{l}\text { Evaporator Vent Gas } \\
\text { cooler Cooling Water } \\
\text { Outlet Temperature } \\
\end{array}$ & Operating : $97^{\circ} \mathrm{F}$ & $\begin{array}{l}\text { System Design. } \\
\text { V-135A-004-123 }\end{array}$ \\
\hline TW & $\begin{array}{r}-60 \mathrm{I} 128 \\
(88987) \\
\end{array}$ & $\begin{array}{l}\text { Evaporator Vent Gas } \\
\text { Cooler Cooling } \\
\text { Water Inlet } \\
\text { Temperature } \\
\end{array}$ & Operating : $76^{\circ} \mathrm{F}$ & $\begin{array}{l}\text { System Design. } \\
\text { V-135A-004-123 }\end{array}$ \\
\hline $\begin{array}{l}\text { PI } \\
\text { (Local) }\end{array}$ & $\begin{array}{r}-601129 \\
(88987) \\
\end{array}$ & $\begin{array}{l}\text { Evaporator Vent Gas } \\
\text { Cooler Vent Pressure }\end{array}$ & Operating: $\quad 0.5 \mathrm{psig}$ & $\begin{array}{l}\text { System Design. } \\
\text { V-135A-004-123 } \\
\end{array}$ \\
\hline TI & $\begin{array}{r}-60 I 130 \\
(88987) \\
\end{array}$ & $\begin{array}{l}\text { Secondary Waste } \\
\text { Inlet Temperature }\end{array}$ & Operating : $\quad-86^{\circ} \mathrm{F}$ & $\begin{array}{l}\text { System Design. } \\
\text { V-135A-004-123 }\end{array}$ \\
\hline TI & $\begin{array}{r}-60 I 131 \\
(88987) \\
\end{array}$ & $\begin{array}{l}\text { Distillate Flash Tank } \\
\text { Outlet temperature }\end{array}$ & Operating : $213^{\circ} \mathrm{F}$ & $\begin{array}{l}\text { System Design. } \\
\text { V-135A-004-123 }\end{array}$ \\
\hline LIC & $\begin{array}{r}-601132 \\
(89335) \\
\end{array}$ & $\begin{array}{l}\text { Level Control Tank } \\
\text { Level }\end{array}$ & $\begin{array}{lcl}\text { AH: } & 13 \% & \text { Alarm } \\
\text { SV: } & 10 \% & \\
\text { AL: } & 2 \% & \text { Alarm }\end{array}$ & $\begin{array}{l}\text { System Design/As Built. } \\
\text { V-135A-004-123 }\end{array}$ \\
\hline PI & $\begin{array}{r}-601133 \\
(89335) \\
\end{array}$ & $\begin{array}{l}\text { Heater Vapor } \\
\text { Pressure }\end{array}$ & Operating: 14.5 psig & $\begin{array}{l}\text { System Design. } \\
\text { V-135A-004-123 }\end{array}$ \\
\hline PI & $\begin{array}{r}-60 \mathrm{I} 134 \\
(89335) \\
\end{array}$ & $\begin{array}{l}\text { Concentrate Transfer } \\
\text { Pump Discharge } \\
\text { Pressure }\end{array}$ & Operating: 70 psig & $\begin{array}{l}\text { System Design. } \\
\text { V-135A-004-123 }\end{array}$ \\
\hline $\begin{array}{l}\text { AI } \\
(\mathrm{pH})\end{array}$ & $\begin{array}{r}-601135 \\
(89335)\end{array}$ & $\begin{array}{l}\text { Evaporator } \\
\text { Concentrate pH }\end{array}$ & 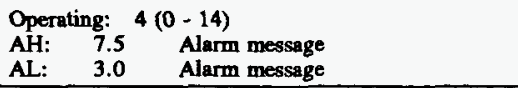 & $\begin{array}{l}\text { System Process Design. } \\
\text { V-135A-004-123 }\end{array}$ \\
\hline
\end{tabular}


TABLE 17 60I WASTE EVAPORATION (Evaporator System)

\begin{tabular}{|c|c|c|c|c|}
\hline \multicolumn{2}{|c|}{$\begin{array}{l}\text { Tag No. (PID No. } \\
\text { H-2-89337) }\end{array}$} & \multirow{2}{*}{$\begin{array}{l}\text { Name } \\
\begin{array}{l}\text { Vapor compressor } \\
\text { Outlet Silencer Level }\end{array} \\
\end{array}$} & Set-Values and Functions & \multirow{2}{*}{$\begin{array}{l}\text { Remarks } \\
\text { System Design. } \\
\text { V-135A-004-123 }\end{array}$} \\
\hline LS & $\begin{array}{r}-601136 \\
(88987) \\
\end{array}$ & & ON Open AOV-60I210 for 5 seconds & \\
\hline $\begin{array}{l}\text { TS } \\
\text { (Local) }\end{array}$ & $\begin{array}{r}-601137 \\
(88987) \\
\end{array}$ & $\begin{array}{l}\text { Vapor Compressor } \\
\text { Temperature }\end{array}$ & $\begin{array}{l}330^{\circ} \mathrm{F} \quad \text { Alarm } \\
\text { Stop Vapor Compressor }\end{array}$ & $\begin{array}{l}\text { Protect Compressor from High Temperature. } \\
\text { V-135A-004-123 }\end{array}$ \\
\hline $\begin{array}{l}\text { PDS } \\
\text { (Local) }\end{array}$ & $\begin{array}{l}-601138 \\
(88987)\end{array}$ & $\begin{array}{l}\text { Vapor Compressor } \\
\text { Differential Pressure }\end{array}$ & $\begin{array}{l}19.5 \text { psi Alarm } \\
\text { Stop Vapor Compressor }\end{array}$ & $\begin{array}{l}\text { Protect Compressor. } \\
\text { V-135A-004-123 }\end{array}$ \\
\hline $\begin{array}{l}\text { TDS } \\
\text { (Local) }\end{array}$ & $\begin{array}{r}-601139 \\
(88987) \\
\end{array}$ & $\begin{array}{l}\text { Vapor Compressor } \\
\text { Differential } \\
\text { Temperature }\end{array}$ & $\begin{array}{l}120^{\circ} \mathrm{F} \quad \text { Alarm } \\
\text { Stop Vapor Compressor }\end{array}$ & $\begin{array}{l}\text { Protect Compressor. } \\
\text { V-135A-004-123 }\end{array}$ \\
\hline $\begin{array}{l}\text { PS } \\
\text { (Local) }\end{array}$ & $\begin{array}{r}601140 \\
(88987) \\
\end{array}$ & $\begin{array}{l}\text { Vapor Compressor } \\
\text { Pressure }\end{array}$ & $\begin{array}{lr}\text { AL: } & -10 \text { inch } \mathrm{H}_{2} \mathrm{O} \text { Alarm } \\
\text { Stop Vapor Compressor }\end{array}$ & $\begin{array}{l}\text { Prevent Damage due to Moisture Carnyover. } \\
\text { V-135A-004-123 }\end{array}$ \\
\hline $\begin{array}{l}\text { PS } \\
\text { (Local) }\end{array}$ & $\begin{array}{r}-601141 \\
(88987) \\
\end{array}$ & $\begin{array}{l}\text { Lube Oil System } \\
\text { Pressure }\end{array}$ & $\begin{array}{l}16 \mathrm{psig}\left({ }^{*}\right) \text { Stop Auxiliary oil pump 2025E-60I-P-7 } \\
13 \mathrm{psig} \text { Start Auxiliary oil pump 2025-602-P-7 }\end{array}$ & $\begin{array}{l}\text { (*) One contact } \\
\text { SH: OFF (open) when rising } \\
\text { SL: ON (close) when falling }\end{array}$ \\
\hline $\begin{array}{l}\text { PS } \\
\text { (Local) }\end{array}$ & $\begin{array}{r}601142 \\
(88987) \\
\end{array}$ & $\begin{array}{l}\text { Lube Oil System } \\
\text { Pressure }\end{array}$ & $\begin{array}{l}11 \text { psig Alarm } \\
\text { Stop Vapor Compressor }\end{array}$ & $\begin{array}{l}\text { Ensure Adequate Lubrication. } \\
\text { V-135A-004-123 }\end{array}$ \\
\hline $\begin{array}{l}\text { TS } \\
\text { (Local) }\end{array}$ & $\begin{array}{r}-601143 \\
(88987) \\
\end{array}$ & $\begin{array}{l}\text { Lube Oil System } \\
\text { Temperature }\end{array}$ & $\begin{array}{c}140^{\circ} \mathrm{F} \quad \text { Alarm } \\
\text { Stop Vapor Compressor }\end{array}$ & $\begin{array}{l}\text { Protect Compressor. } \\
\text { V-135A-004-123 }\end{array}$ \\
\hline II & $\begin{array}{r}-60 I 143 \\
(88987) \\
\end{array}$ & $\begin{array}{l}\text { Vapor Compressor } \\
\text { Current }\end{array}$ & FLA : $765 \mathrm{~A}$ & $\begin{array}{l}\text { V-135A-004-043 } \\
\text { FLA: Full Load Amperage }\end{array}$ \\
\hline $\begin{array}{l}\text { TI } \\
\text { (Local) }\end{array}$ & $\begin{array}{r}-601144 \\
(88987) \\
\end{array}$ & $\begin{array}{l}\text { Oil Cooler outlet oil } \\
\text { Temperature }\end{array}$ & Operating: $100^{\circ} \mathrm{F}$ & $\begin{array}{l}\text { System Design. } \\
\text { V-135A-004-123 }\end{array}$ \\
\hline FS & $\begin{array}{r}-601145 \\
(88987)\end{array}$ & $\begin{array}{l}\text { Distillate Vapor } \\
\text { Compressor Inlet } \\
\text { Flow }\end{array}$ & $\begin{array}{l}\text { Operating: } 4 \mathrm{gpm} \\
\text { AL: } \quad 0.5 \mathrm{gpm} \quad \text { Alarm } \\
\quad \quad \text { Stop Vapor compressor 2025-E-60I-C-01 }\end{array}$ & $\begin{array}{l}\text { V-135A-004-004 } \\
\text { AL: Protect Compressor. }\end{array}$ \\
\hline LS & $\begin{array}{l}-601146 \\
(88987)\end{array}$ & $\begin{array}{l}\text { Vapor Compressor } \\
\text { Inlet Silencer Level }\end{array}$ & Open AOV-60I262 for 5 seconds & $\begin{array}{l}\text { When level is below SH } \\
\text { AOV-601262 Close } \\
\text { AOV-601264 Open }\end{array}$ \\
\hline $\begin{array}{l}\text { FI } \\
\text { (Local) }\end{array}$ & $\begin{array}{r}-601148 \\
(88987) \\
\end{array}$ & $\begin{array}{l}\text { Distillate Vapor } \\
\text { Compressor Inlet } \\
\text { Flow }\end{array}$ & Operating: $4 \mathrm{gpm}$ & $V-135 A-004-123$ \\
\hline $\begin{array}{l}\text { PI } \\
\text { (Local) }\end{array}$ & $\begin{array}{l}-601149 \\
(88987)\end{array}$ & $\begin{array}{l}\text { Oil Cooler Cooling } \\
\text { water Inlet Pressure }\end{array}$ & Operating : 100 psig & $V-135 A-004-123$ \\
\hline
\end{tabular}


TABLE 17 60I WASTE EVAPORATION (Evaporator System)

\begin{tabular}{|c|c|c|c|c|}
\hline \multicolumn{2}{|c|}{$\begin{array}{l}\text { Tag No. (PID No. } \\
\text { H-2-89337) }\end{array}$} & \multirow{2}{*}{$\begin{array}{l}\text { Name } \\
\text { Concentrate Transfer } \\
\text { Pump Current } \\
\end{array}$} & Set-Values and Functions & \multirow{2}{*}{\begin{tabular}{|l} 
Remarks \\
V-135A-004-043 \\
Full Load Amperage \\
\end{tabular}} \\
\hline II & $\begin{array}{r}-601150 \\
(89335) \\
\end{array}$ & & FLA : $5.3 \mathrm{~A}$ & \\
\hline $\begin{array}{l}\text { PI } \\
\text { (Local) }\end{array}$ & $\begin{array}{r}-601152 \\
(88987) \\
\end{array}$ & Boiler Pressure & Operating : $20 \mathrm{psig}$ & $V-135 A-004-123$ \\
\hline $\begin{array}{l}\text { TI } \\
\text { (Local) }\end{array}$ & $\begin{array}{r}-601153 \\
(88987) \\
\end{array}$ & Boiler Temperature & Operating : $250^{\circ} \mathrm{F}$ & $V-135 A-004-123$ \\
\hline $\begin{array}{l}\text { PI } \\
\text { (Local) }\end{array}$ & $\begin{array}{l}-60 \mathrm{I} 154 \\
(89335) \\
\end{array}$ & $\begin{array}{l}\text { Distillate Reuse } \\
\text { Pressure } \\
\end{array}$ & Operating : 55 psig & V-135A-004-123 \\
\hline $\begin{array}{l}\text { PI } \\
\text { (Local) }\end{array}$ & $\begin{array}{r}-601155 \\
(89335) \\
\end{array}$ & $\begin{array}{l}\text { Distillate/ } \\
\text { Verification Pressure }\end{array}$ & Operating : 50 psig & V-135A-004-123 \\
\hline II & $\begin{array}{r}-601169 \\
(89335) \\
\end{array}$ & $\begin{array}{l}\text { Recirculation Pump } \\
\text { Current }\end{array}$ & FLA: $\quad 89.4 \mathrm{~A}$ & $\begin{array}{l}\text { V-135A-004-123 } \\
\text { FLA: Full Load Amperage }\end{array}$ \\
\hline II & $\begin{array}{r}-601187 \\
(88987) \\
\end{array}$ & $\begin{array}{l}\text { Silencer Drain Pump } \\
\text { Current }\end{array}$ & FLA: $\quad 8.9 \mathrm{~A}$ & $\begin{array}{l}\text { V-135A-004-123 } \\
\text { FLA: Full Lond Amperage } \\
\end{array}$ \\
\hline II & $\begin{array}{r}-601199 \\
(88987) \\
\end{array}$ & $\begin{array}{l}\text { Distillate Flash } \\
\text { Tank Pump Current }\end{array}$ & FLA: $\quad 18.2 \mathrm{~A}$ & $\begin{array}{l}\text { V-135A-004-043 } \\
\text { FLA: Full Lond Ampernge }\end{array}$ \\
\hline $\begin{array}{l}\text { LSL } \\
\text { (local) }\end{array}$ & $\begin{array}{r}-601290 \\
(88987) \\
\end{array}$ & Boiler Level & $\begin{array}{ll}\text { MOV Open: } 57 / 8^{\prime \prime} & \text { Trip: } 31 / 8^{*} \\
\text { MOV Close: } 67 / 8^{\prime \prime} & \text { Reset: } 51 / 4^{\prime \prime}\end{array}$ & Boiler Water Level Control \\
\hline $\begin{array}{l}\text { LSLL } \\
\text { (local) }\end{array}$ & $\begin{array}{r}-601291 \\
(88987) \\
\end{array}$ & Boiler Level & $\begin{array}{cc}\text { ALL: } & \text { Trip: } 31 / 8^{*} \text { Mov Open: } 57 / 8^{*} \\
& \text { Reset: } 51 / 4^{*} \text { Mov Close: } 67 / 8^{\prime \prime} \\
\end{array}$ & Low low water cutoff \\
\hline $\begin{array}{l}\text { PS } \\
\text { (local) }\end{array}$ & $\begin{array}{r}-601292 \\
(88987) \\
\end{array}$ & Boiler Pressure & AHH : $\begin{array}{c}47.5 \text { psig Alarm } \\
\text { Shut down boiler }\end{array}$ & High-high pressure cutoff \\
\hline $\begin{array}{l}\text { PS } \\
\text { (local) }\end{array}$ & $\begin{array}{r}-601293 \\
(88987) \\
\end{array}$ & Boiler Pressure & AH: $\quad 45$ psig Alarm & $V-135 A-004-123$ \\
\hline $\begin{array}{l}\text { PC } \\
\text { (local) }\end{array}$ & $\begin{array}{r}-601294 \\
(88987) \\
\end{array}$ & $\begin{array}{l}\text { Boiler Steam } \\
\text { Pressure }\end{array}$ & SV: $\quad 20 \mathrm{psig}$ & V-135A-004-123 \\
\hline $\begin{array}{l}\text { LG } \\
\text { (Local) }\end{array}$ & $\begin{array}{r}-601303 \\
(88987) \\
\end{array}$ & Boiler Level & & $\begin{array}{l}\text { Tag No. Changed from } 601197 \\
\text { V-135A-004-123 }\end{array}$ \\
\hline II & $\begin{array}{r}-601305 \\
(88987) \\
\end{array}$ & $\begin{array}{l}\text { Aux. Oil Pump } \\
\text { Current }\end{array}$ & FLA : $3.1 \mathrm{~A}$ & $\begin{array}{l}\text { V-135A-004-123 } \\
\text { FLA: Full Load Amperage }\end{array}$ \\
\hline $\begin{array}{l}\text { FI } \\
\text { (Local) }\end{array}$ & $\begin{array}{l}-601805 \\
(89335)\end{array}$ & $\begin{array}{l}\text { Recirculation Pump } \\
\text { Seal Water Flow }\end{array}$ & Operating : $2.5 \mathrm{gPm}$ & $V-135 A-004-123$ \\
\hline
\end{tabular}


TABLE 18 60J WASTE DRYING (Concentrate Receiving System)

\begin{tabular}{|c|c|c|c|c|}
\hline \multicolumn{2}{|c|}{$\begin{array}{l}\text { Tag No. (PID No. } \\
\text { H-2-89337) }\end{array}$} & \multirow{2}{*}{$\begin{array}{l}\text { Name } \\
\begin{array}{l}\text { Concentrate Tank(A) } \\
\text { Level }\end{array}\end{array}$} & Set-Values and Functions & \multirow[t]{2}{*}{ Remarks } \\
\hline LI & $\begin{array}{r}-60 \mathrm{J001A} \\
\mathbf{( 8 8 9 8 8 )} \\
\end{array}$ & & $\begin{array}{cc}\text { AHH: } & 94.8 \% \text { Alarm - Shift positions of } \\
& \text { AOV-60J029 and close AOV-65C041, } \\
& \text { 65C042. If H alarm of LS-60J001B has } \\
\text { already annunciated, Shift Evaporator from } & \text { READY mode to HOT } \\
& \\
\text { AH: } & 92.7 \% \quad \text { Alarm Message } \\
\text { AL: } & 4.4 \% \quad \text { Alarm message } \\
\text { ALL: } & 2.3 \% \quad \text { Alarm - Stop Concentrate Tank } \\
& \text { Pump(A) 2025E-60J-P-1A }\end{array}$ & \\
\hline LI & $\begin{array}{r}-60 \mathrm{~J} 001 \mathrm{~B} \\
\mathbf{( 8 8 9 8 8 )} \\
\end{array}$ & $\begin{array}{l}\text { Concentrate Tank(B) } \\
\text { Level }\end{array}$ & 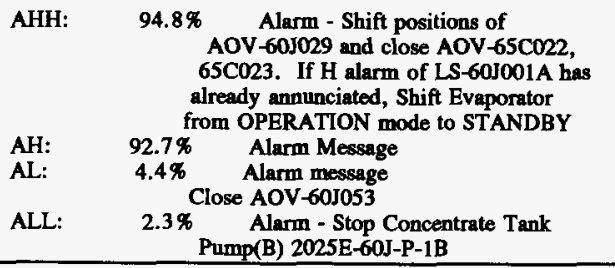 & Same as LI-60J001A \\
\hline $\mathbf{T I}$ & $\begin{array}{r}-60 J 002 A \\
(88988) \\
\end{array}$ & $\begin{array}{l}\text { Concentrate Tank(A) } \\
\text { Temperature }\end{array}$ & $\begin{array}{l}\text { Operating Range: } 60 \sim 230^{\circ} \mathrm{F} \\
\text { Expected temp.: }<140^{\circ} \mathrm{F} \\
\text { AH: } \quad 220^{\circ} \mathrm{F} \text { Alarm }\end{array}$ & $\begin{array}{l}\text { Lower than max. operating temperature } 230^{\circ} \mathrm{F} \\
\text { Prevent Boiling }\end{array}$ \\
\hline TI & $\begin{array}{r}-60 \mathrm{~J} 002 \mathrm{~B} \\
(88988) \\
\end{array}$ & $\begin{array}{l}\text { Concentrate Tank (B) } \\
\text { Temperature }\end{array}$ & $\begin{array}{l}\text { Operating Range: } 60 \sim 230^{\circ} \mathrm{F} \\
\text { Expected temp.: }<140^{\circ} \mathrm{F} \\
\text { AH: } \quad 220^{\circ} \mathrm{F} \text { Alamm }\end{array}$ & Same as TI-60J002A \\
\hline PI & $\begin{array}{r}-601011 A \\
(88988) \\
\end{array}$ & $\begin{array}{l}\text { Concentrate Tank } \\
\text { Pump(A) Discharge } \\
\text { Pressure } \\
\end{array}$ & Operating: $\quad 76$ psig & $\begin{array}{l}\text { SP.GR } 1.407 \\
\text { Head } 125 \mathrm{ft}\end{array}$ \\
\hline PI & $\begin{array}{r}-60 \mathrm{~J} 011 \mathrm{~B} \\
(88988) \\
\end{array}$ & $\begin{array}{l}\text { Concentrate Tank } \\
\text { Pump(B) Discharge } \\
\text { Pressure }\end{array}$ & Same as PI-60J011A & Same as PI-60J011A \\
\hline $\begin{array}{l}\text { AI } \\
\text { (pH) }\end{array}$ & $\begin{array}{r}-60 J 012 A \\
(88988) \\
\end{array}$ & $\begin{array}{l}\text { Concentrate Tank(A) } \\
\text { pH }\end{array}$ & $\begin{array}{lll}\text { Operating Range: } & 3-6 \\
\text { Set Value: } & 4 & \\
\text { AH: } & 7.0 & \text { Alarm } \\
\text { AL: } & 3.5 & \text { Alarm } \\
\end{array}$ & $\begin{array}{l}4(\text { Expected value })+0.5 \\
4(\text { Expected value })-0.5\end{array}$ \\
\hline $\begin{array}{l}\mathbf{A} I \\
(\mathrm{pH})\end{array}$ & $\begin{array}{r}-60 J 012 B \\
(88988) \\
\end{array}$ & $\begin{array}{l}\text { Concentrate Tank(B) } \\
\text { pH }\end{array}$ & Same as AI-60J112A & Same as AI-60J012A \\
\hline
\end{tabular}


TABLE 18 60J WASTE DRYING (Concentrate Receiving System)

\begin{tabular}{|rr|l|l|l|}
\hline \multicolumn{2}{|l|}{$\begin{array}{l}\text { Tag No. (PID No. } \\
\text { H-2-89337) }\end{array}$} & Name & Set-Values and Functions & Remarks \\
\hline II & $\begin{array}{r}-60 \mathrm{J017} \\
(88988)\end{array}$ & $\begin{array}{l}\text { Concentrate } \\
\text { Circulation Pump (B) } \\
\text { Current }\end{array}$ & FLA : 18.2 A & V-1358-002-004 \\
\hline II & -605032 & $\begin{array}{l}\text { Concentrate } \\
\text { Circulation Pump } \\
\text { (A) Current }\end{array}$ & FLA : 18.2 A & V-1358-002-004 \\
FLA: Full Load Amperage
\end{tabular}


TABLE 19 60J WASTE DRYING (Dryer System)

\begin{tabular}{|c|c|c|c|c|}
\hline \multicolumn{2}{|c|}{$\begin{array}{l}\text { Tag No. (PID No. } \\
\text { H-2-89337) }\end{array}$} & \multirow{2}{*}{$\begin{array}{l}\text { Name } \\
\begin{array}{l}\text { Dryer Steam } \\
\text { Pressure }\end{array}\end{array}$} & Set-Values and Functions & \multirow{2}{*}{ R-135A-005 } \\
\hline $\begin{array}{l}\text { PI } \\
\text { (Local) }\end{array}$ & $\begin{array}{l}-60 \mathrm{~J} 004 \\
(88989)\end{array}$ & & Operating : 150 psig & \\
\hline PI & $\begin{array}{l}-60 J 005 \\
(88989)\end{array}$ & $\begin{array}{l}\text { Concentrate Feed } \\
\text { Pump Discharge } \\
\text { Pressure }\end{array}$ & Operating : 10 psig & $V-135 A-005$ \\
\hline PI & $\begin{array}{r}-605007 \\
(88989)\end{array}$ & $\begin{array}{l}\text { Dryer Distillate } \\
\text { Pump Discharge } \\
\text { Pressure } \\
\end{array}$ & Operating : 30 psig & $V-135 A-005$ \\
\hline $\begin{array}{l}\text { PI } \\
\text { (Local) }\end{array}$ & $\begin{array}{l}-60 j 010 \\
(88989) \\
\end{array}$ & $\begin{array}{l}\text { Dryer Seal Water } \\
\text { Pressure }\end{array}$ & Operating : 25 psig & $V-135 A-005$ \\
\hline TI & $\begin{array}{r}-60 j 011 \\
(88989) \\
\end{array}$ & $\begin{array}{l}\text { Dryer Outlet Steam } \\
\text { Temperature }\end{array}$ & $\begin{array}{ll}\text { Operating : } & 365^{\circ} \mathrm{F} \\
\text { SH : } 228^{\circ} \mathrm{F} & \text { End of Warming-up (HEAT-UP 1) }\end{array}$ & V-135A-005 \\
\hline \multirow[t]{2}{*}{ TI } & $\begin{array}{l}-60 j 014 \\
(88989) \\
\end{array}$ & $\begin{array}{l}\text { Dryer Steam } \\
\text { Temperature }\end{array}$ & Operating : $365^{\circ} \mathrm{F}$ & $V-135 A-005$ \\
\hline & $\begin{array}{l}-60 j 018 \\
(88989)\end{array}$ & $\begin{array}{l}\text { Dryer Powder } \\
\text { Hopper Temp }{ }^{\circ} \mathrm{F}\end{array}$ & $\begin{array}{ll}\text { Operating : } & \\
\text { SH: } 212^{\circ} \mathrm{F} & \text { Start Concentrate Feed Pump } \\
\text { AL: } 194^{\circ} \mathrm{F} & \text { Alarm }\end{array}$ & $V-135 A-005$ \\
\hline TI & $\begin{array}{r}-60 \mathrm{~J} 019 \\
(88989) \\
\end{array}$ & $\begin{array}{l}\text { Dryer Vapor } \\
\text { Temperature } \\
\end{array}$ & Operating : $212^{\circ} \mathrm{F}$ & $V-135 A-005$ \\
\hline TS & $\begin{array}{l}-60 \mathrm{~J} 020 \\
(88989) \\
\end{array}$ & Drum Powder Level & SH: $225^{\circ} \mathrm{F}$ & To detoct powder level in the drum (drum fill) \\
\hline TIC & $\begin{array}{l}-605028 \\
(88989)\end{array}$ & $\begin{array}{l}\text { Dryer Feed } \\
\text { Temperature }\end{array}$ & 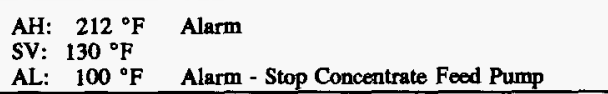 & $\begin{array}{l}\text { System Design. } \\
\text { V-135A-005 }\end{array}$ \\
\hline PIC & $\begin{array}{r}-605032 \\
(88989) \\
\end{array}$ & $\begin{array}{l}\text { Dryer Steam } \\
\text { Pressure }\end{array}$ & $\begin{array}{l}\text { SV2: } 150 \text { psig } \\
\text { SH: } 150 \text { psig Starting condition of Concentrate Feed Pump } \\
\text { SV1: } 10 \text { psig Initial set point } \\
\text { AL: } 135 \text { psig Alarm - Stop Concentrate Feed Pump } \\
\end{array}$ & $\begin{array}{l}\text { System Design. } \\
\text { V-135A-005 }\end{array}$ \\
\hline PIC & $\begin{array}{r}-60 j 033 \\
(88989) \\
\end{array}$ & $\begin{array}{l}\text { Dryer Vapor } \\
\text { Pressure }\end{array}$ & $\begin{array}{l}\text { AH: }+5.0 \text { in } \mathrm{H}_{2} \mathrm{O} \text { Alarm } \\
\text { SV: }-6 \text { in } \mathrm{H}_{2} \mathrm{O}\end{array}$ & $V-135 A-005$ \\
\hline FI & $\begin{array}{r}-60 J 034 \\
(88989)\end{array}$ & Dryer Feed Flow & Operating : $480 \mathrm{lb} / \mathrm{hr}$ & $\begin{array}{l}0.8 \mathrm{gpm} \text { Process Design. } \\
\text { V-135A-005 }\end{array}$ \\
\hline FI & $\begin{array}{l}-60 \mathrm{~J} 035 \\
(88989)\end{array}$ & $\begin{array}{l}\text { Distillate to Surge } \\
\text { Tank Flow }\end{array}$ & Operating : $0.6 \mathrm{gpm}$ & $\begin{array}{l}\text { Process Design. } \\
\text { V-135A-005 }\end{array}$ \\
\hline
\end{tabular}


TABLE 19 60J WASTE DRYING (Dryer System)

\begin{tabular}{|c|c|c|c|c|}
\hline \multicolumn{2}{|c|}{$\begin{array}{l}\text { Tag No. (PID No. } \\
\text { H-2-89337) } \\
\end{array}$} & \multirow{2}{*}{$\begin{array}{l}\text { Name } \\
\begin{array}{l}\text { Spray Condenser } \\
\text { Level }\end{array}\end{array}$} & Set-Values and Functions & \multirow{2}{*}{$\begin{array}{l}\text { Remarks } \\
\text { Range: } 0 \text { - } 24 \text { in water } \\
\text { Design Operation } \\
\text { V-135A-005 }\end{array}$} \\
\hline LIC & $\begin{array}{r}-60 J 036 \\
(88989) \\
\end{array}$ & & $\begin{array}{ll}\text { SH: } 60 \% & \text { Close AOV-60J157 (Weekly Flush) } \\
\text { SV: } 50 \% & \\
\text { AL: } 10 \% & \text { Alarm - Stop dryer distillate Pump } \\
& \text { 2025E-60J-P-3 } \\
\end{array}$ & \\
\hline TI & $\begin{array}{l}-601038 \\
(88989) \\
\end{array}$ & $\begin{array}{l}\text { Distillate Condenser } \\
\text { Outlet Temperature } \\
\end{array}$ & $\begin{aligned} & \text { Operating: } 130^{\circ} \mathrm{F} \\
& \text { AH: } 160^{\circ} \mathrm{F} \text { Alarm } \\
&\end{aligned}$ & $\begin{array}{l}\text { Design Conditions } \\
\text { V-135A-005 }\end{array}$ \\
\hline $\begin{array}{l}\text { FI } \\
\text { (Local) }\end{array}$ & $\begin{array}{l}-60 j 041 \\
(88989) \\
\end{array}$ & Dryer SA Seal Flow & Operating : $3.4 \mathrm{scfh}$ & $\begin{array}{l}\text { Pressure : } 5 \text { psig } \\
\text { V-135A-005-901 }\end{array}$ \\
\hline $\begin{array}{l}\text { FI } \\
\text { (Local) }\end{array}$ & $\begin{array}{r}-60 \mathrm{~J} 042 \\
(88989) \\
\end{array}$ & $\begin{array}{l}\text { Dryer Seal Water } \\
\text { Flow }\end{array}$ & Operating : $1 \mathrm{gpm}$ & $V-135 A-005-901$ \\
\hline (Local) & $\begin{array}{l}-60 \mathrm{J045} \\
(88989) \\
\end{array}$ & $\begin{array}{l}\text { Vent Cooler Cooling } \\
\text { Water Outlet } \\
\text { Temperature } \\
\end{array}$ & Operating : $94^{\circ} \mathrm{F}$ & $V-135 A-005$ \\
\hline $\begin{array}{l}\text { PI } \\
\text { (Local) }\end{array}$ & $\begin{array}{l}-60 \mathrm{~J} 048 \\
(88989)\end{array}$ & $\begin{array}{l}\text { Dryer Vacuum } \\
\text { Blower Inlet } \\
\text { Pressure }\end{array}$ & Operating : $0-10$ in $\mathrm{H}_{2} \mathrm{O}$ & $V-135 A-005$ \\
\hline II & $\begin{array}{l}-605055 \\
(88989)\end{array}$ & $\begin{array}{l}\text { Dryer Distillate } \\
\text { Pump Current }\end{array}$ & FLA: $6.2 \mathrm{~A}$ & FLA: Full Load Amperage \\
\hline II & $\begin{array}{l}-60 \mathrm{J056} \\
(88989)\end{array}$ & Dryer Current & FLA: $21.7 \mathrm{~A}$ & FLA: Fult Load Amperage \\
\hline $\begin{array}{l}\text { TI } \\
\text { (Local) }\end{array}$ & $\begin{array}{l}-60 \mathrm{~J} 194 \\
(88989)\end{array}$ & $\begin{array}{l}\text { Hot Water } \\
\text { Temperature }\end{array}$ & Operating: $180-200^{\circ} \mathrm{F}$ & $\begin{array}{l}\text { Temperature Range during Weekly Flush. } \\
\text { V-135A-005 }\end{array}$ \\
\hline $\begin{array}{l}\text { PI } \\
\text { (Local) }\end{array}$ & $\begin{array}{l}-60 \mathrm{~J} 200 \\
(88989) \\
\end{array}$ & $\begin{array}{l}\text { Dryer Seal SA } \\
\text { Pressure }\end{array}$ & Operating : 5 psig & V-135A-005 \\
\hline $\begin{array}{l}\text { PI } \\
\text { (Local) }\end{array}$ & $\begin{array}{l}-60 \mathrm{~J} 201 \\
(88989)\end{array}$ & $\begin{array}{l}\text { Dryer Foed } \\
\text { Preheater Steam } \\
\text { Pressure 1 }\end{array}$ & Operating : 15 psig & $V-135 A-005$ \\
\hline $\begin{array}{l}\text { PI } \\
\text { (Local) }\end{array}$ & $\begin{array}{l}-60 J 202 \\
(88989) \\
\end{array}$ & $\begin{array}{l}\text { Dryer Feed } \\
\text { Preheater Steam } \\
\text { Pressure } 2\end{array}$ & Operating : 15 psig & $V-135 A-005$ \\
\hline $\begin{array}{l}\text { TI } \\
\text { (Local) }\end{array}$ & $\begin{array}{l}-601203 \\
(88989)\end{array}$ & $\begin{array}{l}\text { Distillate Condenser } \\
\text { Cooling Water Outlet } \\
\text { Temperature }\end{array}$ & Operating : $86^{\circ} \mathrm{F}$ & $v-135 A-005$ \\
\hline
\end{tabular}


TABLE 19 60J WASTE DRYING (Dryer System)

\begin{tabular}{|c|c|c|c|c|}
\hline \multicolumn{2}{|c|}{$\begin{array}{l}\text { Tag No. (PID No. } \\
\text { H-2-89337) }\end{array}$} & Name & Set-Values and Functions & Remarks \\
\hline $\begin{array}{l}\text { TI } \\
\text { (Local) }\end{array}$ & $\begin{array}{l}-60 J 204 \\
(88989) \\
\end{array}$ & $\begin{array}{l}\text { Distillate Condenser } \\
\text { Cooling Water Inlet } \\
\text { Temperature }\end{array}$ & Operating : $76^{\circ} \mathrm{F}$ & $V-135 A-005$ \\
\hline RI & $\begin{array}{l}-60 \mathrm{~J} 233 \\
(88989)\end{array}$ & $\begin{array}{l}\text { Filled Drum Area } \\
\text { Radiation }\end{array}$ & Operating : $2.3 * 10^{1} \mathrm{mk} / \mathrm{h}, 3 \mathrm{ft}$ from Drum Storage Area & $2.1^{\mathrm{mk}} / \mathrm{kr} 3 \mathrm{ft}$ from Drum Storage Area \\
\hline
\end{tabular}


TABLE $20 \quad 80 \mathrm{C}$ DRUM HANDLING SYSTEM

\begin{tabular}{|c|c|c|c|}
\hline $\begin{array}{l}\text { Tag No. (PID No. } \\
\text { H-2-89337) }\end{array}$ & Name & Set-Values and Functions & Remarks \\
\hline $\begin{array}{r}-80 C 400 \\
(88989)\end{array}$ & Drum Weight & Operating : 417.3 pounds & $\begin{array}{l}\text { Approximate Full Weight. Control Based on } \\
\text { Temperature. V-135A-002 }\end{array}$ \\
\hline
\end{tabular}

TABLE 21 1B COMPRESSED AIR SYSTEM (Service Air)

\begin{tabular}{|c|c|c|c|c|}
\hline \multicolumn{2}{|c|}{$\begin{array}{l}\text { Tag No. (PID No. } \\
\text { H-2-89337) }\end{array}$} & \multirow{2}{*}{$\begin{array}{l}\text { Name } \\
\text { Service Air } \\
\text { Receiving Tank } \\
\text { Pressure }\end{array}$} & Set-Values and Functions & \multirow{2}{*}{ Remarks } \\
\hline $\begin{array}{l}\text { PI } \\
\text { (Local) }\end{array}$ & $\begin{array}{r}-1 \mathrm{BO01} \\
(89000)\end{array}$ & & Operating: $110 \mathrm{psig}$ & \\
\hline PI & $\begin{array}{r}-1 \mathrm{~B} 011 \\
(89000) \\
\end{array}$ & Service Air Pressure & $\begin{array}{l}\text { Operating: } 90 \text { psig } \\
\text { AL: } \quad 80 \mathrm{psig}\end{array}$ & Process Design Vahue. \\
\hline II & $\begin{array}{r}-1 \mathrm{B031} \\
(89000)\end{array}$ & Compressor Current & FLA : 178 A & Full Load Amperage \\
\hline
\end{tabular}

TABLE 22 1D COMPRESSED AIR SYSTEM (Instrument Air)

\begin{tabular}{|c|c|c|c|c|}
\hline \multicolumn{2}{|c|}{$\begin{array}{l}\text { Tag No. (PID No. } \\
\text { H-2-89337) }\end{array}$} & Name & Set-Values and Functions & Remarks \\
\hline $\begin{array}{l}\text { PI } \\
\text { (Local) }\end{array}$ & $\begin{array}{l}-1 \mathrm{D} 001 \\
(89336)\end{array}$ & $\begin{array}{l}\text { Instrument Air } \\
\text { Receiving Tank } \\
\text { Pressure }\end{array}$ & Operating: $110 \mathrm{psig}$ & Process Design Value. \\
\hline PI & $\begin{array}{l}-1 \mathrm{D} 011 \\
(89336)\end{array}$ & $\begin{array}{l}\text { Instrument Air } \\
\text { Pressure }\end{array}$ & $\begin{array}{l}\text { Operating: } 90 \text { psig } \\
\text { AL: } \quad 80 \text { psig }\end{array}$ & $\begin{array}{l}\text { Operating pressure of Air-operated valve is } 90 \\
\text { psig }\end{array}$ \\
\hline
\end{tabular}


TABLE 23 20B SUMP \& BUILDING DRAINS (Sump Tank System)

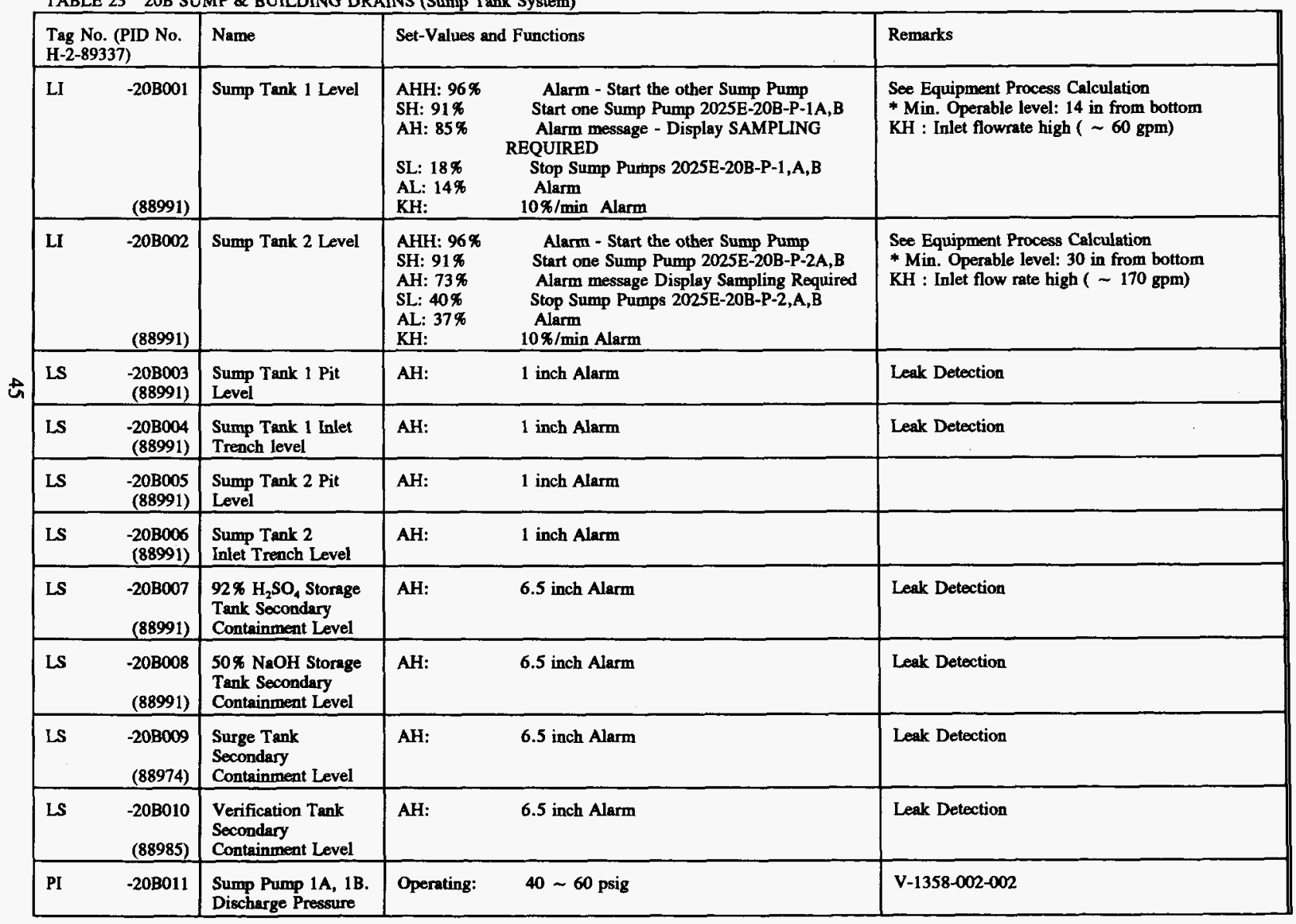


TABLE 23 20B SUMP \& BUILDING DRAINS (Sump Tank System)

\begin{tabular}{|c|c|c|c|c|}
\hline \multicolumn{2}{|c|}{$\begin{array}{l}\text { Tag No. (PID No. } \\
\text { H-2-89337) }\end{array}$} & \multirow{2}{*}{$\begin{array}{l}\text { Name } \\
\begin{array}{l}\text { Sump Pump 2A, 2B. } \\
\text { Discharge Pressure }\end{array}\end{array}$} & Set-Values and Functions & \multirow{2}{*}{$\frac{\text { Remarks }}{\text { V-1358-002-002 }}$} \\
\hline PI & $\begin{array}{r}-20 \mathrm{~B} 012 \\
(\mathbf{8 8 9 9 1 )} \\
\end{array}$ & & $40 \sim 60$ psig & \\
\hline PI & $\begin{array}{r}-20 \mathrm{~B} 013 \\
(88991)\end{array}$ & $\begin{array}{l}\text { Sump Tank } 1 \text { Inlet } \\
\text { Trench Level }\end{array}$ & 1 inch Alarm & Leak Detection \\
\hline LS & $\begin{array}{r}-20 \mathrm{~B} 014 \\
(88991) \\
\end{array}$ & $\begin{array}{l}\text { Sump Tank } 1 \text { Inlet } \\
\text { Trench Level }\end{array}$ & 1 inch Alarm & Leak Detection \\
\hline LS & $\begin{array}{r}-20 \mathrm{~B} 015 \\
(89350) \\
\end{array}$ & $\begin{array}{l}\mathrm{H}_{2} \mathrm{O}_{2} \text { Feed Tank } \\
\text { Secondary } \\
\text { Containment Level }\end{array}$ & 1 inch Alarm & Leak Detection \\
\hline LS & $\begin{array}{r}-20 \mathrm{~B} 016 \\
(88992)\end{array}$ & $\begin{array}{l}4 \% \mathrm{H}_{2} \mathrm{SO}_{4} / \mathrm{NaOH} \\
\text { Tank Secondary } \\
\text { Containment Level }\end{array}$ & 1 inch Alarm & Leak Detection \\
\hline $\begin{array}{l}\text { AI } \\
\text { (COND) }\end{array}$ & $\begin{array}{r}-20 \mathrm{~B} 021 \\
(88991) \\
\end{array}$ & $\begin{array}{l}\text { Sump Tank } 1 \\
\text { Conductivity }\end{array}$ & Operating Range: $0-1500 \mathrm{E}-6 \mathrm{~S} / \mathrm{cm}$ & Assume as same as receiving line conductivity \\
\hline FI & $\begin{array}{r}-20 \mathrm{~B} 022 \\
(88991) \\
\end{array}$ & $\begin{array}{l}\text { Sump Tank } 1 \text { Outlet } \\
\text { Flow }\end{array}$ & Operating Range: $30-60 \mathrm{gpm}$ & Design Process Value. \\
\hline $\begin{array}{l}\text { AI } \\
\text { (COND) }\end{array}$ & $\begin{array}{r}-20 \mathrm{~B} 024 \\
(88991) \\
\end{array}$ & $\begin{array}{l}\text { Sump Tank } 2 \\
\text { conductivity }\end{array}$ & Operating range: $0-1500 \mathrm{E}-6 \mathrm{~S} / \mathrm{cm}$ & Design Process Value. \\
\hline $\mathrm{FI}$ & $\begin{array}{r}-20 \mathrm{~B} 025 \\
(88991) \\
\end{array}$ & $\begin{array}{l}\text { Sump Tank } 2 \text { Outlet } \\
\text { Flow }\end{array}$ & Operating Range: $100-200$ gpm & Design Process Value. \\
\hline II & $\begin{array}{r}-20 \mathrm{~B} 032 \\
(88991) \\
\end{array}$ & $\begin{array}{l}\text { Sump Pump 1A } \\
\text { Current } \\
\end{array}$ & $10.2 \mathrm{~A}$ & $\begin{array}{l}\text { V-1358-002-325 } \\
\text { FLA: Full Load Amperage }\end{array}$ \\
\hline II & $\begin{array}{r}-20 \mathrm{~B} 034 \\
(88991) \\
\end{array}$ & $\begin{array}{l}\text { Sump Pump 1B } \\
\text { Current }\end{array}$ & $10.2 \mathrm{~A}$ & $\begin{array}{l}\text { V-1358-002-325 } \\
\text { FLA: Full Load Amperage }\end{array}$ \\
\hline II & $\begin{array}{r}-20 \mathrm{~B} 036 \\
(88991) \\
\end{array}$ & $\begin{array}{l}\text { Sump Pump 2A } \\
\text { Current }\end{array}$ & $12.5 \mathrm{~A}$ & $\begin{array}{l}\text { V-1358-002-330 } \\
\text { FLA: Full Load Amperage }\end{array}$ \\
\hline II & $\begin{array}{r}-20 \mathrm{~B} 038 \\
(88991)\end{array}$ & $\begin{array}{l}\text { Sump Pump 2B } \\
\text { Current }\end{array}$ & $12.5 \mathrm{~A}$ & $\begin{array}{l}\text { V-1358-002-330 } \\
\text { FLA: Full Load Amperage }\end{array}$ \\
\hline
\end{tabular}


TABLE 24 45D PROCESS OFFGAS (Vessel Ventilation System)

\begin{tabular}{|c|c|c|c|}
\hline $\begin{array}{l}\text { Tag No. (PID No. } \\
\text { H-2-89337) }\end{array}$ & \multirow{2}{*}{$\begin{array}{l}\text { Name } \\
\begin{array}{l}\text { VOG Filter Unit } \\
\text { Inlet Pressure }\end{array}\end{array}$} & Set-Values and Functions & \multirow{2}{*}{$\begin{array}{l}\text { Remarks } \\
\text { Process Design Value. }\end{array}$} \\
\hline $\begin{array}{r}-45 \mathrm{D} 001 \\
(88993) \\
\end{array}$ & & $\begin{array}{lll}\mathrm{AH}: 0^{\prime \prime} & \mathrm{H}_{2} \mathrm{O} & \text { Alarm } \\
\text { SV }: 4^{\prime \prime} & \mathrm{H}_{2} \mathrm{O} & \\
\text { AL }:-8.8^{\prime \prime} & \mathrm{H}_{2} \mathrm{O} & \text { Alarm } \\
\end{array}$ & \\
\hline $\begin{array}{r}-45 D 003 \\
(88993) \\
\end{array}$ & $\begin{array}{l}\text { VOG Blower } \\
\text { Discharge } \\
\text { Temperature } \\
\end{array}$ & Operating Range: $60^{\circ}-170^{\circ} \mathrm{F}$ & $V-1358-004$ \\
\hline $\begin{array}{r}-45 \mathrm{D} 005 \\
(88993) \\
\end{array}$ & $\begin{array}{l}\text { VOG Blower } \\
\text { Discharge Flow }\end{array}$ & Operating Range: $0-900 \mathrm{cfm}$ & See Equipment Process Calculation \\
\hline $\begin{array}{r}-45 D 032 \\
(88993) \\
\end{array}$ & $\begin{array}{l}\text { VOG Blower (A) } \\
\text { Current }\end{array}$ & FLA: $6.1 \mathrm{~A}$ & FLA: Full Load Amperage \\
\hline $\begin{array}{r}-45 \mathrm{D} 034 \\
(88993) \\
\end{array}$ & $\begin{array}{l}\text { VOG Blower (B) } \\
\text { Current }\end{array}$ & FLA: $6.1 \mathrm{~A}$ & FLA: Full Laad Amperage \\
\hline $\begin{array}{l}\text { 45D101 } \\
\text { (88993) } \\
\end{array}$ & $\begin{array}{l}\text { VOG Filter HEPA } \\
\text { Filter Differential } \\
\text { Pressure }\end{array}$ & Operating : $1.0^{\prime \prime}-2.0^{\prime \prime} \mathrm{H}_{2} \mathrm{O}$ & $\begin{array}{l}V-1358-004-301 \\
\text { CSC letter on } 6 / 10 / 94\end{array}$ \\
\hline $\begin{array}{lr}\text { PDI } & -45 D 102 \\
\text { (LOCAL) } & (88993) \\
\end{array}$ & $\begin{array}{l}\text { VOG Filter Demister } \\
\text { Differential Pressure }\end{array}$ & Operating : $1.0^{N} \sim 1.5^{\mathrm{N}} \mathrm{H}_{2} \mathrm{O}$ & V-1358-004-301 \\
\hline $\begin{array}{ll}\text { PDI } & -45 D 103 \\
\text { (LOCAL) } & (88993) \\
\end{array}$ & $\begin{array}{l}\text { VOG Filter Pre- } \\
\text { Filter Differential } \\
\text { Pressure }\end{array}$ & Operating : $0.1 \sim 1.0^{n} \mathrm{H}_{2} \mathrm{O}$ & $V-1358-004-301$ \\
\hline $\begin{array}{r}-45 \mathrm{D} 105 \\
(88993) \\
\end{array}$ & $\begin{array}{l}\text { VOG Filter HEPA } \\
\text { Filter Differential } \\
\text { Pressure }\end{array}$ & $1.0^{\prime \prime} \sim 2.0^{\prime \prime} \mathrm{H}_{2} \mathrm{O}$ & $V-1358-004-301$ \\
\hline $\begin{array}{r}-45 D 106 \\
(88993) \\
\end{array}$ & $\begin{array}{l}\text { VOG Filter Heater } \\
\text { Outlet Temperature }\end{array}$ & $\begin{array}{lll}\text { SH: } & 135{ }^{\circ} \mathrm{F} & \begin{array}{c}\text { Heater off } \\
\text { SL: }\end{array} \\
\end{array}$ & Design Process Value. \\
\hline $\begin{array}{r}-45 \mathrm{D} 107 \\
(88993)\end{array}$ & $\begin{array}{l}\text { VOG Filter Charcoal } \\
\text { Filter Outlet } \\
\text { Temperature }\end{array}$ & $\begin{array}{l}\text { Operating: } \\
\text { AH: }\end{array}$ & $V-1358-004-301$ \\
\hline
\end{tabular}




\begin{tabular}{|c|c|c|c|c|}
\hline \multicolumn{2}{|c|}{$\begin{array}{l}\text { Tag No. (PID No. } \\
\text { H-2-89337) }\end{array}$} & \multirow{2}{*}{ Name } & Set-Values and Functions & \multirow{2}{*}{$\begin{array}{l}\text { Remarks } \\
\text { Range: } 0 \text { - } 8 \text { in } \\
\text { Design Operating Levels } \\
\text { V-135A-012 }\end{array}$} \\
\hline LI & $\begin{array}{r}-65 A 001 \\
(88999) \\
\end{array}$ & & $\begin{array}{ll}\text { AH }: 7.5 \text { in } & \text { Alarm } \\
\text { SH }: 4 \text { in } & \text { Close Demineralized water feed valve } \\
\text { SL }: 2.66 \text { in } & \text { Open Demineralized water feed valve } \\
\text { AL }: 1 \text { in } & \text { Alarm - Stop Feed Pump }\end{array}$ & \\
\hline PI & $\begin{array}{r}-65 A 002 \\
(88999) \\
\end{array}$ & $\begin{array}{l}\text { Feed Pump } \\
\text { Discharge Pressure }\end{array}$ & Operating : $170 \mathrm{psig}$ & $V-135 A-012$ \\
\hline PI & $\begin{array}{r}-65 A 003 \\
(88999) \\
\end{array}$ & $\begin{array}{l}\text { Condensate Tank } \\
\text { Pressure }\end{array}$ & 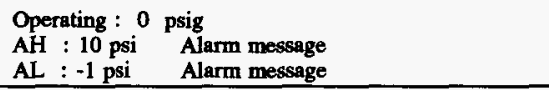 & $V-135 A-012$ \\
\hline II & $\begin{array}{r}-65 A 004 \\
(88999) \\
\end{array}$ & Feed Pump Current & FLA: $4 \mathrm{~A}$ & FLA: Full Load Amperage \\
\hline LI & $\begin{array}{r}-65 A 010 \\
(88999) \\
\end{array}$ & Boiler Level & $\begin{array}{l}\text { AH }: 6 \text { in Alarm } \\
\text { SH }: 4.25 \text { in Stop Feed Pump } \\
\text { SL }: 3.33 \text { in Start Feed Pump } \\
\text { AL }: 1.75 \text { in Alarm Stop Boiler }\end{array}$ & $\begin{array}{l}\text { Range: } 0-8 \text { in } \\
\text { V-135A-012-307 } \\
\text { Design Operating Levels }\end{array}$ \\
\hline $\begin{array}{l}\text { PIC } \\
\text { PC }\end{array}$ & $\begin{array}{r}-65 A 011 \\
(88999) \\
-65 A 041 \\
(88999) \\
\end{array}$ & $\begin{array}{l}\text { Boiler Pressure } \\
\text { (remote) } \\
\text { Boiler Pressure } \\
\text { (local) }\end{array}$ & $\begin{array}{l}\text { Operating : } 155 \text { psi } \\
\text { AH : } 168 \text { psig Alarm Stop Boiler } \\
\text { SV: } 155 \text { psig } \\
\text { AL : } 140 \text { psig Alarm }\end{array}$ & V-135A-012-307 and as Built. \\
\hline LS & $\begin{array}{r}-65 A 013 \\
(88999) \\
\end{array}$ & Boiler Level & $\begin{array}{r}\text { AL: } \mathbf{0 . 7 5} \text { in (*) } \\
\text { Stop Boiler }\end{array}$ & 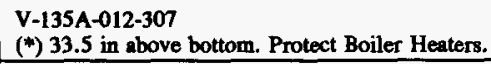 \\
\hline PS & $\begin{array}{r}-65 \mathrm{~A} 014 \\
(88999) \\
\end{array}$ & Boiler Pressure & $\begin{array}{c}\text { AH: } 170 \text { psig } \\
\text { Stop Boiler }\end{array}$ & $\begin{array}{l}\text { V-135A-012-307 } \\
\text { Protect Boiler from Over Pressure } \\
\end{array}$ \\
\hline TIC & $\begin{array}{r}-65 A 035 \\
(88999)\end{array}$ & $\begin{array}{l}\text { Condensate Tank } \\
\text { Temperature }\end{array}$ & SV: $205 \mathrm{~F}$ & $V-135 A-012$ \\
\hline
\end{tabular}


TABLE 26 65C CHEMICAL MAKEUP AND STORAGE (Chemical Reagent Feed System)

\begin{tabular}{|c|c|c|c|c|c|}
\hline \multicolumn{2}{|c|}{\begin{tabular}{|l|} 
Tag No. (PID No. \\
H-2-89337) \\
\end{tabular}} & \multirow{2}{*}{$\begin{array}{l}\text { Name } \\
\begin{array}{l}92 \% \mathrm{H}_{2} \mathrm{SO}_{4} \text { Storage } \\
\text { Tank Level }\end{array} \\
\end{array}$} & \multicolumn{2}{|c|}{ Set-Values and Functions } & \multirow[t]{2}{*}{ Remarks } \\
\hline $\mathrm{LI}$ & $-65 \mathrm{C} 101$ & & $\begin{array}{l}\text { AH : } 94 \% \\
\text { AL : } 8 \% \\
\text { ALL : } 5 \%\end{array}$ & $\begin{array}{l}\text { Alarm } \\
\text { Alarm Message - Display } \mathrm{H}_{2} \mathrm{SO}_{4} \text { SUPPLY } \\
\text { REQUIRED } \\
\text { Alarm - Stop 92\% } \mathrm{H}_{2} \mathrm{SO}_{4} \text { Storage Tank Pump } \\
\text { 2025E-60C-P-1 }\end{array}$ & \\
\hline PI & $\begin{array}{r}-65 C 102 \\
(88992) \\
\end{array}$ & $\begin{array}{l}92 \% \mathrm{H}_{2} \mathrm{SO}_{4} \text { Storage } \\
\text { Tank Pump Discharge } \\
\text { Pressure }\end{array}$ & Operating: $60 \mathrm{ps}$ & & $\begin{array}{l}\text { Head } 77 \mathrm{ft} \\
\text { Specific Gravity } 1.82(77 \mathrm{~F})\end{array}$ \\
\hline FQI & $\begin{array}{r}-65 C 103 \\
(88992) \\
\end{array}$ & $\begin{array}{l}92 \% \mathrm{H}_{2} \mathrm{SO}_{4} \text { Integrated } \\
\text { Flow }\end{array}$ & $\begin{array}{l}\text { Operating Flow } \\
\text { SH }: 2.2 \text { gal } \\
\text { (FQS) }\end{array}$ & $\begin{array}{l}\text { rate : } 0.63 \mathrm{gpm} \\
\text { Close AOV-65C-010 }\end{array}$ & Flowrate $0.63 \mathrm{gpm}$ set by HV-65C006 \\
\hline FQI & (88992) & \begin{tabular}{|l|} 
Verification Tank \\
Effluent Integrated \\
Flow
\end{tabular} & $\begin{array}{l}\text { SAH : } 89.5 \mathrm{gal} \\
\text { SHH : } 162.6 \mathrm{ga} \\
\text { AL : } 16 \mathrm{gpm}\end{array}$ & $\begin{array}{l}\text { When AOV-65C052 is opened by LSL- } \\
65 \mathrm{C} 111 \text {, close AOV-65C052 } \\
\text { When AOV-65C051 is opened by } \\
\text { LSL-65C211, close AOV-65C051 } \\
\text { Alarm (for } \mathrm{H}_{2} \mathrm{SO}_{4} \text { preparation) } \\
\text { Close AOV-65C010 }\end{array}$ & $\begin{array}{ll}\text { * For } \mathrm{H}_{2} \mathrm{SO}_{4} \text { preparation, flowrate is set to } \\
24.0 \mathrm{gpm} \text { by HV-65C-086 } \\
\text { * } \quad \text { For } \mathrm{NaOH} \text { Preparation, flowrate is set to } \\
23.2 \mathrm{gpm} \text { by HV-65C-087 }\end{array}$ \\
\hline LS & $\begin{array}{r}-65 C 105 \\
(88992) \\
\end{array}$ & $\begin{array}{l}92 \% \mathrm{H}_{2} \mathrm{SO}_{4} \text { Storage } \\
\text { Tank Level }\end{array}$ & AH : $96 \%$ & Alarm & $\begin{array}{l}\text { Back-up for LI-65C101 } \\
\mathrm{H}+2 \%\end{array}$ \\
\hline LI & $-65 C 111$ & $\begin{array}{l}4 \mathrm{O} \mathrm{H}_{2} \mathrm{SO}_{4} \text { Solution } \\
\text { Tank Level }\end{array}$ & $\begin{array}{l}\text { AHH: } 96 \% \\
\text { AH : } 93 \% \\
\text { SL : } 73 \% \\
\text { AL : } 5 \%\end{array}$ & $\begin{array}{l}\text { Alarm - Stop } 92 \% \mathrm{H}^{2} \mathrm{SO}^{4} \text { Storage Tank Pump } \\
\text { Alarm } \\
\text { Open AOV-65C010, } 65 \mathrm{CO52} \\
\text { Start-up condition of polisher regeneration } \\
\text { operation } \\
\text { Alarm - Stop } 4 \% \mathrm{H}_{2} \mathrm{SO}_{4} \text { Solution Tank Pump } \\
2025 \mathrm{E}-65 \mathrm{C}-\mathrm{P}-3\end{array}$ & $\begin{array}{l}\text { See Equipment Process Calculation } \\
\text { Polisher Regeneration } \\
\text { (V-135A-006-010) } \\
537 \text { gal } \\
\text { keep more than } 270 \text { gal between SL and AL }\end{array}$ \\
\hline PI & $\begin{array}{r}-65 \mathrm{C} 112 \\
(88992)\end{array}$ & $\begin{array}{l}4 \mathrm{H}_{2} \mathrm{H}_{2} \mathrm{SO}_{4} \text { Solution } \\
\text { Tank Pump Discharge } \\
\text { Pressure }\end{array}$ & Operating: $66 \mathrm{ps}$ & & Specific Gravity 1.02 (77 F) \\
\hline TI & (88992) & $\begin{array}{l}92 \% \mathrm{H}_{2} \mathrm{SO}_{4} \text { Storage } \\
\text { Tank Temperature }\end{array}$ & $\begin{array}{l}\text { Operating : }-15^{\circ} \\
\mathrm{AH}: 110^{\circ} \mathrm{F} \\
\mathrm{AL}:-15^{\circ} \mathrm{F}\end{array}$ & $\begin{array}{l}\text { F } 110^{\circ} \mathrm{F} \\
\text { Alarm } \\
\text { Alarm }\end{array}$ & $\begin{array}{l}\text { Freezing point: }-15^{\circ} \mathrm{F}(92 \text { wt } \%) \\
\quad\left(-4^{\circ} \mathrm{F}(90 \text { wt } \%)\right) \\
\text { Tank heater control set point: } 25^{\circ} \mathrm{F} \\
\quad \text { (thermostat) } \\
\text { SH: } 25^{\circ} \mathrm{F} \text { Turn off heater } \\
\text { SL: } 15^{\circ} \mathrm{F} \text { Tum on heater }\end{array}$ \\
\hline$\pi$ & $\begin{array}{r}-65 C 122 \\
(88992)\end{array}$ & $\begin{array}{l}4 \% \mathrm{H}_{2} \mathrm{SO}_{4} \text { Solution } \\
\text { Tank Temperature }\end{array}$ & $\begin{array}{l}\text { Operating : } 60 \\
\mathrm{AH}: 110^{\circ} \mathrm{F}\end{array}$ & $\begin{array}{l}110^{\circ} \mathrm{F} \\
\text { Alarm - Close AOV-65C010, } 65 \mathrm{CO} 22\end{array}$ & Lower than max. operating temperature $120^{\circ} \mathrm{F}$ \\
\hline LS & $\begin{array}{r}-65 C 143 \\
(88984)\end{array}$ & $\begin{array}{l}\mathrm{H}_{2} \mathrm{SO}_{4} \text { Dilution Pot } \\
\text { Level }\end{array}$ & $A L: O N$ & Alarm & Prevent Operation without $\mathrm{H} 2 \mathrm{SO} 4$ \\
\hline
\end{tabular}


TABLE 26 65C CHEMICAL MAKEUP AND STORAGE (Chemical Reagent Feed System)

\begin{tabular}{|c|c|c|c|c|c|}
\hline \multicolumn{2}{|c|}{$\begin{array}{l}\text { Tag No. (PID No. } \\
\text { H-2-89337) }\end{array}$} & \multirow{2}{*}{\begin{tabular}{|l|} 
Name \\
Secondary Waste \\
Receiving Tank $\mathrm{H}_{2} \mathrm{SO}_{4}$ \\
Integrated Flow \\
\end{tabular}} & \multicolumn{2}{|c|}{ Set-Values and Functions } & \multirow{2}{*}{\begin{tabular}{|l|} 
Remarks \\
Set value will be calculated by the operator. \\
\end{tabular}} \\
\hline$\overline{F I}$ & $\begin{array}{r}-65 \mathrm{C} 144 \\
(88986)\end{array}$ & & \multicolumn{2}{|c|}{ SH : $(22 \sim 52) \mathrm{gal}$} & \\
\hline FI & $\begin{array}{r}-65 \mathrm{C} 145 \\
(88988)\end{array}$ & $\begin{array}{l}\text { Concentrate Tank } \\
\mathrm{H}_{2} \mathrm{SO}_{4} \text { Integrated Flow }\end{array}$ & \multicolumn{2}{|c|}{ SH : $(360 \sim 1850) c c$} & Set value will be calculated by the operator. \\
\hline FIC & $\begin{array}{r}-65 \mathrm{C} 146 \\
(88983)\end{array}$ & $\begin{array}{l}\text { Polisher } \mathrm{H}_{2} \mathrm{SO}_{4} \\
\text { Integrated Flow }\end{array}$ & \multicolumn{2}{|c|}{$\begin{array}{l}\text { AH : } 21 \mathrm{gpm} \text { Alarm } \\
\text { SV : } 17.9 \mathrm{gpm} \\
\text { AL : } 16 \mathrm{gpm} \text { Alarm }\end{array}$} & V-135A-006-017 \\
\hline II & $\begin{array}{r}-65 C 155 \\
(88992)\end{array}$ & \begin{tabular}{|l|}
$92 \% \mathrm{H}_{2} \mathrm{SO}_{4}$ \\
Storage Tank \\
Pump Current
\end{tabular} & \multicolumn{2}{|c|}{ FLA : $10.2 \mathrm{~A}$} & FLA: Full Load Amperage \\
\hline II & $\begin{array}{r}-65 \mathrm{C} 156 \\
(88982)\end{array}$ & \begin{tabular}{|l}
$4 \% \mathrm{H}_{2} \mathrm{SO}_{4}$ \\
Solution Tank \\
Pump Current
\end{tabular} & \multicolumn{2}{|c|}{ FLA : $12.5 \mathrm{~A}$} & FLA: Full Load Amperage \\
\hline $\mathrm{LI}$ & (88992) & $\begin{array}{l}50 \% \mathrm{NaOH} \text { Storage } \\
\text { Tank Level }\end{array}$ & $\begin{array}{l}\text { AH : } 96 \% \\
\text { AL : } 12 \% \\
\text { ALL : } 6 \%\end{array}$ & $\begin{array}{l}\text { Alarm } \\
\text { Alarm } \\
\text { Display "NaOH SUPPLY REQUIRED" } \\
\text { Alarm - Stop 50\% NaOH Storage Tank Pump } \\
\text { 202SE-60C-P-2 }\end{array}$ & Process Design Values \\
\hline PI & $\begin{array}{r}-65 \mathrm{C} 202 \\
(88992)\end{array}$ & $\begin{array}{l}\text { 50\% NaOH Storage } \\
\text { Tank Pump Discharge } \\
\text { Pressure }\end{array}$ & \multicolumn{2}{|c|}{ Operating : 50 psig } & $\begin{array}{l}\text { Head: } 77 \mathrm{ft} \\
\text { Specific Gravity: } 1.52 \text { (77 F) }\end{array}$ \\
\hline FQI & $\begin{array}{r}-65 C 203 \\
(88992)\end{array}$ & $\begin{array}{l}50 \% \mathrm{NaOH} \text { Integrated } \\
\text { Flow }\end{array}$ & \multicolumn{2}{|c|}{$\begin{array}{l}\text { Operating flow rate: } 1.4 \mathrm{gpm} \\
\text { SH: } 9.4 \mathrm{gal} \quad \text { Close AOV-65C-030, 65C051 }\end{array}$} & Flowrate is set to $1.4 \mathrm{gpm}$ by HV-65C029 \\
\hline LS & $\begin{array}{r}-65 C 204 \\
(88992)\end{array}$ & $\begin{array}{l}\text { 50\% NaOH Storage } \\
\text { Tank Level }\end{array}$ & \multicolumn{2}{|c|}{ AH: $98 \% \quad$ Alarm } & $\begin{array}{l}\text { Back-up for LI-65C201 } \\
\mathrm{H}+2 \%\end{array}$ \\
\hline LI & (88992) & $\begin{array}{l}4 \% \mathrm{NaOH} \text { Solution } \\
\text { Tank Level }\end{array}$ & $\begin{array}{l}\text { AHH: } 98 \% \\
\text { AH : } 95 \% \\
\text { SL }: 72 \% \\
\text { AL : } 20 \%\end{array}$ & $\begin{array}{l}\text { Alarm - Stop } 50 \% \text { NaOH Storage Tank Pump } \\
\text { Alarm - Close AOV-65C030, 65C-051 } \\
\text { Open AOV-65C030, 65C051. Start-up } \\
\text { condition of polisher regeneration operation } \\
\text { Stop } 4 \% \text { NaOH Solution Tank Pump 2025E- } \\
65 \mathrm{C}-\mathrm{P}-4\end{array}$ & $\begin{array}{l}\text { See equipment Process Calculation } \\
\text { Polisher regeneration : (V-135A-006-010) } \\
\text { Caustic Exhaustion } 90 \mathrm{gal} \\
\text { Caustic Intro. } 528 \mathrm{gal} \\
\text { Caustic/Acid Intro. } 528 \mathrm{gal} \\
\text { Keep } 573 \mathrm{gal} \text { between SL and AL } \\
\end{array}$ \\
\hline PI & $\begin{array}{r}-65 C 212 \\
(88992)\end{array}$ & $\begin{array}{l}4 \% \text { NaOH Solution } \\
\text { Tank Pump Discharge } \\
\text { Pressure }\end{array}$ & \multicolumn{2}{|c|}{ Operating : 63 psig } & Specific Gravity: 1.04 (77 F) \\
\hline TI & $\begin{array}{r}-65 C 221 \\
(88992)\end{array}$ & $\begin{array}{l}50 \% \mathrm{NaOH} \text { Storage } \\
\text { Tank Temperature }\end{array}$ & \multicolumn{2}{|c|}{$\begin{array}{l}\text { AH : } 110^{\circ} \mathrm{F} \text { Alarm } \\
\text { SH : } 85^{\circ} \mathrm{F} \text { HEATER OFF } \\
\text { SL : } 75^{\circ} \mathrm{F} \text { HEATER ON } \\
\text { AL : } 70^{\circ} \mathrm{F} \text { Alarm }\end{array}$} & 50 wt $\% \mathrm{NaOH}$ solubility limit: $66^{\circ} \mathrm{F}$ \\
\hline
\end{tabular}


TABLE 26 65C CHEMICAL MAKEUP AND STORAGE (Chemical Reagent Feed System)

\begin{tabular}{|c|c|c|c|c|}
\hline \multicolumn{2}{|c|}{$\begin{array}{l}\text { Tag No. (PID No. } \\
\text { H-2-89337) }\end{array}$} & \multirow{2}{*}{\begin{tabular}{|l|} 
Name \\
$4 \%$ NaOH Solution \\
Tank Temperature \\
\end{tabular}} & \multirow{2}{*}{\begin{tabular}{|l|} 
Set-Values and Functions \\
Operating Range : $0-120^{\circ} \mathrm{F}$ \\
$\mathrm{AH}: 110^{\circ} \mathrm{F} \quad$ Alarm \\
\end{tabular}} & \multirow{2}{*}{\begin{tabular}{|l|} 
Remarks \\
Process Design Value. \\
\end{tabular}} \\
\hline TI & $\begin{array}{r}-65 C 222 \\
(88992)\end{array}$ & & & \\
\hline LS & $\begin{array}{r}-65 C 243 \\
(88984)\end{array}$ & $\begin{array}{l}\text { NaOH Dilution Pot } \\
\text { Level }\end{array}$ & $\mathrm{AL}: \mathrm{ON} \quad$ Alarm & Prevent Operation without $\mathrm{NAOH}$ \\
\hline FI & $\begin{array}{r}-65 C 244 \\
(88986) \\
\end{array}$ & $\begin{array}{l}\text { Secondary Waste } \\
\text { Receiving Tank NaOH } \\
\text { Integrated Flow }\end{array}$ & SH $:(2 \sim 72) \mathrm{gal}$ & Set value will be calculated by the operator \\
\hline FI & $\begin{array}{r}-65 C 245 \\
(88988)\end{array}$ & $\begin{array}{l}\text { Concentrate Tank } \\
\text { NaOH Integrated Flow }\end{array}$ & $\mathrm{SH}: 0.249 \sim 1.27 \mathrm{gal}$ & Set value will be calculated by the operator \\
\hline FIC & $\begin{array}{r}-65 C 246 \\
(88938) \\
\end{array}$ & $\begin{array}{l}\text { Polisher } \mathrm{NaOH} \\
\text { Integrated Flow }\end{array}$ & $\begin{array}{|ll|}\text { AH : } 21 \mathrm{gpm} & \text { Alarm } \\
\text { SV : } 17.6 \mathrm{gpm} & \\
\text { AL : } 16 \mathrm{gpm} & \text { Alarm } \\
\end{array}$ & V-135A-006-017 \\
\hline II & $\begin{array}{r}-65 C 255 \\
(88992)\end{array}$ & $\begin{array}{l}50 \% \text { NaOH Storage } \\
\text { Tank Pump Current }\end{array}$ & FLA : $10.2 \mathrm{~A}$ & FLA: Full Load Amperage \\
\hline II & $\begin{array}{r}-65 C 256 \\
(88992)\end{array}$ & $\begin{array}{l}\text { 4\% NaOH Solution } \\
\text { Tank Pump Current }\end{array}$ & FLA : $12.5 \mathrm{~A}$ & FLA: Full Load Amperage \\
\hline
\end{tabular}


TABLE 27 95B CLOSED LOOP COOLING (Seal Water System)

\begin{tabular}{|c|c|c|c|c|}
\hline \multicolumn{2}{|c|}{$\begin{array}{l}\text { Tag No. (PID No. } \\
\text { H-2-89337) }\end{array}$} & \multirow{2}{*}{$\begin{array}{l}\text { Name } \\
\begin{array}{l}\text { Seal Water Tank } \\
\text { Level }\end{array}\end{array}$} & \multirow{2}{*}{$\begin{array}{l}\text { Set-Values and Functions } \\
\text { AHH : } 95 \% \text { Alarm } \\
\text { AL : } 10 \% \text { Alarm } \\
\text { ALL : } 5 \% \text { Alarm Stop Seal water pump 2025E-95B-P-1 }\end{array}$} & \multirow{2}{*}{$\begin{array}{l}\text { Remarks } \\
\text { Process Design Values. }\end{array}$} \\
\hline LI & $\begin{array}{r}-95 \mathrm{B001} \\
(89001) \\
\end{array}$ & & & \\
\hline PI & $\begin{array}{r}-95 \mathrm{~B} 002 \\
(89001) \\
\end{array}$ & $\begin{array}{l}\text { Seal Water Pump } \\
\text { Discharge Pressure }\end{array}$ & $\begin{array}{l}\text { Operating : } 80 \text { psig } \\
\text { AL : } 65 \text { psig Alarm }\end{array}$ & Process Design Values. \\
\hline $\begin{array}{l}\text { TI } \\
\text { (Local) }\end{array}$ & $\begin{array}{r}-95 \mathrm{~B} 021 \\
(89001) \\
\end{array}$ & $\begin{array}{l}\text { Seal Water cooler } \\
\text { Outlet Temperature }\end{array}$ & Operating : $<90^{\circ} \mathrm{F}$ & Process Design Values \\
\hline II & $\begin{array}{r}-958032 \\
(89001)\end{array}$ & $\begin{array}{l}\text { Seal Water Pump } \\
\text { Current }\end{array}$ & FLA : $2.2 \mathrm{~A}$ & $\begin{array}{l}\text { V-135A-015-303 } \\
\text { FLA: Full Load Amperage }\end{array}$ \\
\hline
\end{tabular}


TABLE 28 95C COOLING WATER (Cooling Water System)

\begin{tabular}{|c|c|c|c|c|}
\hline \multicolumn{2}{|c|}{$\begin{array}{l}\text { Tag No. (PID No. } \\
\text { H-2-89337) }\end{array}$} & \multirow{2}{*}{\begin{tabular}{|l|} 
Name \\
$\begin{array}{l}\text { Cooling Water } \\
\text { Pressure }\end{array}$
\end{tabular}} & \multirow{2}{*}{\begin{tabular}{|l|} 
Set-Values and Functions \\
Operating: 80 psig \\
AL : 70 psig Alarm
\end{tabular}} & \multirow{2}{*}{$\begin{array}{l}\text { Remarks } \\
\begin{array}{l}\text { Maintain following condition to avoid cross } \\
\text { contamination. Cooling water pressure }>\text { Process } \\
\text { stream (Max: } 70 \text { psig) }\end{array}\end{array}$} \\
\hline PI & $\begin{array}{r}-950012 \\
(88998)\end{array}$ & & & \\
\hline II & $\begin{array}{r}-95 C 032 \\
(88998) \\
\end{array}$ & $\begin{array}{l}\text { Cooling Water Pump } \\
\text { Current }\end{array}$ & FLA : $110 \mathrm{~A}$ & FLA: Fuil Load Amperage \\
\hline \begin{tabular}{|l} 
FI \\
(Local)
\end{tabular} & $\begin{array}{r}-95 C 041 \\
(88998) \\
\end{array}$ & $\begin{array}{l}\text { Air Compressor Unit } \\
\text { Cooling Water Flow }\end{array}$ & Operating : $28 \mathrm{gpm}$ & V-135A-011-316 \\
\hline $\begin{array}{l}\text { FI } \\
\text { (Local) }\end{array}$ & $\begin{array}{r}-95 C 042 \\
(88998)\end{array}$ & $\begin{array}{l}\text { Seal Water Cooler } \\
\text { Cooling Water Flow }\end{array}$ & Operating : $9 \mathrm{gpm}$ & V-135A-015-304 \\
\hline $\begin{array}{l}\text { FI } \\
\text { (Local) }\end{array}$ & $\begin{array}{r}-95 \mathrm{CO} 43 \\
(88998)\end{array}$ & \begin{tabular}{|l|} 
Evaporator \\
Compressor Oil \\
Cooler Cooling Water \\
Flow
\end{tabular} & Operating : $33 \mathrm{gpm}$ & V-135A-015-123 \\
\hline $\begin{array}{l}\text { FI } \\
\text { (Local) }\end{array}$ & $\begin{array}{r}-95 \mathrm{C} 044 \\
(88998)\end{array}$ & $\begin{array}{l}\text { Evaporator Vent Gas } \\
\text { Cooler Cooling Water } \\
\text { Flow }\end{array}$ & Operating : $93 \mathrm{gpm}$ & V-135A-015-123 \\
\hline $\begin{array}{l}\text { FI } \\
\text { (Local) }\end{array}$ & $\begin{array}{r}-950046 \\
(88998)\end{array}$ & $\begin{array}{l}\text { Surge Tank Pump (C) } \\
\text { Cooling Water Flow }\end{array}$ & Operating : $6 \mathrm{gpm}$ & $\mathrm{V}-1358-002-\mathrm{B}-001$ \\
\hline $\begin{array}{l}\mathbf{F I} \\
\text { (Local) }\end{array}$ & \begin{tabular}{r|}
$-95 C 047$ \\
$(88998)$
\end{tabular} & $\begin{array}{l}\text { Surge Tank Pump (B) } \\
\text { Cooling Water Flow }\end{array}$ & Operating : $6 \mathrm{gpm}$ & Same as Surge Tank Pump (A) \\
\hline \begin{tabular}{|l} 
FI \\
(Local)
\end{tabular} & $\begin{array}{r}-95 \mathrm{CO48} \\
(88998)\end{array}$ & $\begin{array}{l}\text { Surge Tank Pump (A) } \\
\text { Cooling Water Flow }\end{array}$ & Operating : $6 \mathrm{gpm}$ & Same as Surge Tank Pump (A) \\
\hline $\begin{array}{l}\text { FI } \\
\text { (Local) }\end{array}$ & $\begin{array}{r}-95 C 049 \\
(88998)\end{array}$ & $\begin{array}{l}\text { Degasification Column } \\
\text { Inlet Cooler Cooling } \\
\text { Water Flow }\end{array}$ & Operating : $261 \mathrm{gpm}$ & Process Design Value. \\
\hline $\begin{array}{l}\text { FI } \\
\text { (Local) }\end{array}$ & $\begin{array}{r}-95 \mathrm{C} 050 \\
(88998)\end{array}$ & $\begin{array}{l}\text { UV Oxidation Inlet } \\
\text { Cooler Cooling Water } \\
\text { Flow }\end{array}$ & Operating : $192 \mathrm{gpm}$ & Process Design Value. \\
\hline FI & $\begin{array}{r}-95 \operatorname{Co51} \\
(88998)\end{array}$ & $\begin{array}{l}\text { Cooling Water Blow } \\
\text { Down Flow }\end{array}$ & Operating : $22 \mathrm{gpm}$ & Process Design Value. \\
\hline $\begin{array}{l}\text { FI } \\
\text { (Local) }\end{array}$ & $\begin{array}{r}-95 C 052 \\
(88998)\end{array}$ & $\begin{array}{l}\text { Secondary Waste Feed } \\
\text { Pump (B) Cooling } \\
\text { Water Flow }\end{array}$ & Operating: $2 \mathrm{gpm}$ & $\mathrm{V}-1358-002-\mathrm{B}-003$ \\
\hline $\begin{array}{l}\text { FI } \\
\text { (Local) }\end{array}$ & $\begin{array}{r}-95 C 053 \\
(88998)\end{array}$ & $\begin{array}{l}\text { Secondary Waste Feed } \\
\text { Pump (A) Cooling } \\
\text { Water Flow }\end{array}$ & Operating : $2 \mathrm{gpm}$ & Same as Secondary Waste Feed Pump (B) \\
\hline
\end{tabular}


TABLE 28 95C COOLING WATER (Cooling Water System)

\begin{tabular}{|c|c|c|c|c|}
\hline \multicolumn{2}{|c|}{\begin{tabular}{|l} 
Tag No. (PID No. \\
H-2-89337)
\end{tabular}} & \multirow{2}{*}{\begin{tabular}{|l|} 
Name \\
Concentrate \\
Circulation Pump (B) \\
Cooling Water Flow \\
\end{tabular}} & Set-Values and Functions & \multirow{2}{*}{\begin{tabular}{|l|} 
Remarks \\
V-1358-002-004
\end{tabular}} \\
\hline $\begin{array}{l}\text { FI } \\
\text { (Local) }\end{array}$ & $\begin{array}{r}-95 \operatorname{Co54} \\
(88998)\end{array}$ & & Opersting: $2 \mathrm{gpm}$ & \\
\hline $\begin{array}{l}\text { FI } \\
\text { (Local) }\end{array}$ & $\begin{array}{l}-95 \operatorname{Co55} \\
(88998)\end{array}$ & $\begin{array}{l}\text { Concentrate } \\
\text { Circulation Pump (A) } \\
\text { Cooling Water Flow }\end{array}$ & Operating : $2 \mathrm{gpm}$ & Same as Concentrate Circulation Pump (B) \\
\hline $\begin{array}{l}\text { FI } \\
\text { (Local) }\end{array}$ & $\begin{array}{r}-95 \cos 6 \\
(88998)\end{array}$ & $\begin{array}{l}\text { Dryer Boiler cooling } \\
\text { Water Flow }\end{array}$ & Operating : $4 \mathrm{gpm}$ & V-135A-004-123 \\
\hline $\begin{array}{l}\text { FI } \\
\text { (Local) }\end{array}$ & $\begin{array}{l}-95 \mathrm{C} 058 \\
(88998)\end{array}$ & $\begin{array}{l}\text { Dryer condensate } \\
\text { Cooler Cooling Water } \\
\text { Flow }\end{array}$ & Operating : $73 \mathrm{gpm}$ & $V-135 A-005-901$ \\
\hline $\begin{array}{l}\text { FI } \\
\text { (Local) }\end{array}$ & $\begin{array}{l}-95 \operatorname{Cos} 9 \\
(88998)\end{array}$ & $\begin{array}{l}\text { Concentrate Tanks } \\
\text { Vent Gas cooler } \\
\text { Cooling Water Flow }\end{array}$ & Operating : $3 \mathrm{gpm}$ & Process Design Value \\
\hline PI & $\begin{array}{r}-95 C 080 \\
(88998)\end{array}$ & $\begin{array}{l}\text { Blowdown Pump } \\
\text { Outlet Pressure }\end{array}$ & Operating: 85 psig & Process Design Value \\
\hline FI & $\begin{array}{r}-95 \mathrm{C083} \\
(88998)\end{array}$ & $\begin{array}{l}\text { Evaporator Skid } \\
\text { Pumps Cooling Water } \\
\text { Flow }\end{array}$ & Operating: $3.5 \mathrm{gpm}$ & $\begin{array}{l}\text { Approximately value for reference } \\
\text { Distillate Flush Tank Pump } 2 \mathrm{gpm} \\
\text { Silencer Drain Pump } 1 \mathrm{gpm} \\
\text { Concentrate Transfer Pump } 0.5 \mathrm{gpm} \\
\end{array}$ \\
\hline LS & $\begin{array}{r}-95 C 101 \\
(88998) \\
\end{array}$ & Cooling Tower Level & \begin{tabular}{|ll} 
AL : OFF & $\begin{array}{l}\text { Alarm - Stop Cooling Water Pump } \\
\text { 2025E-95C-P-1 }\end{array}$ \\
\end{tabular} & $12.5^{\prime \prime}$ from bottom \\
\hline II & $\begin{array}{r}-95 \mathrm{C} 102 \\
(88998) \\
\end{array}$ & $\begin{array}{l}\text { Cooling Tower (A) } \\
\text { Current }\end{array}$ & FLA : $48 \mathrm{~A}$ & \begin{tabular}{|l|} 
V-135A-010-003 \\
FLA: Full Losd Amperage \\
\end{tabular} \\
\hline$\overline{\mathrm{II}}$ & $\begin{array}{r}-95 C 103 \\
(88998) \\
\end{array}$ & $\begin{array}{l}\begin{array}{l}\text { Cooling Tower (B) } \\
\text { Current }\end{array} \\
\end{array}$ & FLA : $48 \mathrm{~A}$ & $\begin{array}{l}\text { V-135A-010-003 } \\
\text { FLA: Full Load Amperage }\end{array}$ \\
\hline TS & $\begin{array}{r}-95 \mathrm{C} 104 \\
(88998)\end{array}$ & $\begin{array}{l}\text { Cooling Water } \\
\text { Temperature }\end{array}$ & $\begin{array}{ll}\text { Operating: } & 76^{\circ} \mathrm{F} \\
\text { SH2: } 81^{\circ} \mathrm{F} & \text { Fan 2 on } \\
\text { SH1: } 78^{\circ} \mathrm{F} & \text { Fan 1 on }\end{array}$ & Reset Point: 3F below set point \\
\hline TS & \begin{tabular}{r|}
$-95 \mathrm{C} 115$ \\
$(88998)$
\end{tabular} & $\begin{array}{l}\text { Cooling Water } \\
\text { Temperature } \\
\end{array}$ & $\begin{array}{l}\text { Operating temperature: } 40^{\circ} \mathrm{F} \\
\text { AL : } 40^{\circ} \mathrm{F} \text { Heater-on/off }\end{array}$ & thermostat \\
\hline II & $\begin{array}{r}-95 \mathrm{C} 118 \\
(88998)\end{array}$ & $\begin{array}{l}\text { Blowdown Pump } \\
\text { Current }\end{array}$ & FLA : $10.2 \mathrm{~A}$ & FLA: Full Load Amperage \\
\hline $\begin{array}{l}\text { PI } \\
\text { (Local) }\end{array}$ & $\begin{array}{r}-95 \mathrm{C} 122 \\
(88975)\end{array}$ & $\begin{array}{l}\text { UV Inlet cooler } \\
\text { Outlet Pressure }\end{array}$ & Operating : 100 psig & Process Design Value \\
\hline
\end{tabular}


TABLE 29 95D DEMINERALIZED WATER SYSTEM

\begin{tabular}{|lr|l|l|l|}
\hline $\begin{array}{l}\text { Tag No. (PID No. } \\
\text { H-2-89337) }\end{array}$ & Name & Set-Values and Functions & Remarks \\
\hline $\begin{array}{l}\text { PI } \\
\text { (Local) }\end{array} \quad \begin{aligned} \text { (890013) } \\
\text { AI }\end{aligned}$ & $\begin{array}{l}\text { Demineralizer Inlet } \\
\text { Pressure }\end{array}$ & Operating: $50 \mathrm{psig}$ & Process Design Value \\
\hline (89023) & $\begin{array}{l}\text { Demineralizer } \\
\text { Outlet Resistivity }\end{array}$ & $\begin{array}{l}\text { Operating : } 1 \sim 10 \mathrm{MO} / \mathrm{cm} \\
\text { AL: } 1 \mathrm{MM} / \mathrm{cm} \quad \text { Alarm }\end{array}$ & Process Design Value \\
\hline
\end{tabular}


TABLE $30 \quad 45$ HVAC - RCA

\begin{tabular}{|c|c|c|c|}
\hline $\begin{array}{l}\text { Tag No. PID No. } \\
\mathrm{H}-2-89021(1 / 2)\end{array}$ & Name & Set-Values and Functions & Selection Basis \\
\hline FIC-45E001 & $\begin{array}{l}\text { EXHAUST AIR EFFLUENT } \\
\text { DISCHARGE AIR FLOW } \\
\end{array}$ & $\begin{array}{l}\text { OPERATING: } 52,000 \text { CFM } \\
\text { SV: PDIG-45A002 } \\
\end{array}$ & (DoC. C-13K1-001, V-45) \\
\hline II -45A001A & EXHAUST FAN $45 \mathrm{~A}-\mathrm{F}-1 \mathrm{~A}$ & OPERATING: $87 \mathrm{~A}$ & $\begin{array}{l}\text { FULL LOAD AMPERE: 112A (DOC. C-13K1-001, } \\
\text { V-47A) }\end{array}$ \\
\hline II $-45 \mathrm{~A} 001 \mathrm{~B}$ & EXHAUST FAN $45 \mathrm{~A}-\mathrm{F}-1 \mathrm{~B}$ & OPERATING: $87 \mathrm{~A}$ & $\begin{array}{l}\text { FULL LOAD AMPERE: } 112 \mathrm{~A} \text { (DOC C-13K1-001, V- } \\
\text { 47A) }\end{array}$ \\
\hline II -45A001C & EXHAUST FAN $45 \mathrm{~A}-\mathrm{F}-1 \mathrm{C}$ & OPERATING: $87 \mathrm{~A}$ & $\begin{array}{l}\text { FULL LOAD AMPERE: 112A (DOC C-13K1-001, V- } \\
\text { 47A) }\end{array}$ \\
\hline II-45B001A & $\begin{array}{l}\text { AIR HANDLING UNIT } \\
45 \mathrm{~B}-\text { EVU-1A }\end{array}$ & OPERATING: 49 amps & FULL LOAD AMPERE: $57.5 \mathrm{~A}$ \\
\hline II -45B001B & $\begin{array}{l}\text { AIR HANDLING UNIT } \\
45 \mathrm{~B}-\text { EVU-1B }\end{array}$ & OPERATING: 52 amps & FULL LOAD AMPERE: $57.5 \mathrm{~A}$ \\
\hline II $-45 B 006$ & $\begin{array}{l}\text { AIR HANDLING UNIT } \\
45 \mathrm{~B}-\text { EVU-9 }\end{array}$ & OPERATING: 3.2 amps & FULL LOAD AMPERE: $4.1 \mathrm{~A}$ \\
\hline II - 45B007. & $\begin{array}{l}\text { AIR HANDLING UNIT } \\
45 \mathrm{~B}-\mathrm{EVU}-10\end{array}$ & OPERATING: 3.1 & FULL LOAD AMPERE: $4.1 \mathrm{~A}$ \\
\hline PDI - 45A001A & NORTH BLDG EXTERIOR & OPERATING: -0.05 IN & RANGE: $-0.8 \sim+0.2$ IN \\
\hline PDI $-45 A 001 B$ & SOUTH BLDG EXTERIOR & OPERATING : -0.05 IN & RANGE: $-0.8-+0.2$ IN \\
\hline PDI - 45A001C & WEST BLDG EXTERIOR & OPERATING: -0.05 IN & RANGE: $-0.8-+0.2$ IN \\
\hline PDI - 45A001D & EAST BLDG EXTERIOR & OPERATING: -0.05 IN & RANGE: $-0.8 \sim+0.2$ IN \\
\hline PDI - 45A004 & $\begin{array}{l}\text { ROOM } 131 / 113 \\
\text { PRESSURE }\end{array}$ & OPERATING: -0.05 IN & RANGE: $-0.3-+0.2 \mathrm{IN}$ \\
\hline PDAH $-45 A 005$ & $\begin{array}{l}\text { DRYER/DRUM HANDLING } \\
\text { AREA PRESSURE }\end{array}$ & $\begin{array}{l}\text { OPERATING: }-0.1 \text { IN } \\
\mathrm{AH}=0.0 \text { IN }\end{array}$ & RANGE: $-0.3 \sim+0.2$ IN \\
\hline PDI $=45 A 007$ & $\begin{array}{l}\text { ROOM } 131 / 206 \\
\text { PRESSURE }\end{array}$ & OPERATING: $0.05 \mathrm{IN}$ & RANGE: $-0.3 \sim+0.2 \mathrm{IN}$ \\
\hline PDIC-45A002 & Exhaust Fan Control & $\begin{array}{l}\text { SV: }-0.05 \text { IN } \\
\text { AL: }-0.5 \text { IN } \mathrm{H}_{2} \mathrm{O}\end{array}$ & SHUT DOWN RCA HVAC SYSTEM \\
\hline PDI - 45B001A & $\begin{array}{l}\text { AIR HANDLING UNIT } \\
\text { EVU-1A } \\
\text { PRE-FILTER PDI } \\
\end{array}$ & OPERATING: $1.0 \mathrm{IN}$ & (DOC. C-13K1-001,V-44) \\
\hline
\end{tabular}


TABLE $30 \quad 45$ HVAC - RCA

\begin{tabular}{|c|c|c|c|}
\hline $\begin{array}{l}\text { Tag No. PID No. } \\
\text { H-2-89021(1/2) }\end{array}$ & Name & Set-Values and Functions & Selection Basis \\
\hline PDI - 45B001B & $\begin{array}{l}\text { AIR HANDLING UNIT } \\
\text { EVU-1B } \\
\text { PRE-FILTER PDI }\end{array}$ & OPERATING: $1.0 \mathrm{IN}$ & (DOC. C-13K1-001,V-44) \\
\hline PDI $-45 B 008$ & $\begin{array}{l}\text { AIR HANDLING UNIT } \\
\text { 45B-EVU-9 FILTER PDI }\end{array}$ & OPERATING: 1.6 IN & (DOC. C-13K1-001, X-17) \\
\hline PDI - 45B009 & $\begin{array}{l}\text { AIR HANDLING UNIT } \\
\text { 45B-EVU-10 FILTER } \\
\text { PDI }\end{array}$ & OPERATING: $1.6 \mathrm{IN}$ & (DOC. C-13K1-001, X-17) \\
\hline PDI - 45B010A & $\begin{array}{l}\text { AIR HANDLING UNIT } \\
\text { 45B-EVU-1A HEPA } \\
\text { FILTER PDI }\end{array}$ & OPERATING: 2.5 IN & (DOC. C-13K1-001, V-44) \\
\hline PDI - 45B010B & $\begin{array}{l}\text { AIR HANDLING UNIT } \\
\text { 45B - EVU-1B HEPA } \\
\text { FILTER PDI }\end{array}$ & OPERATING: 2.5 IN & (DOC. C-13K1-001, V-44) \\
\hline PDI - 45C001A & $\begin{array}{l}\text { HEPA FILTER 45C-FL- } \\
\text { 2A PRE-FILTER PDI } \\
\end{array}$ & OPERATING: 1.2 IN & (DOC. C-13K1-001, V-46) \\
\hline PDI $-45 \mathrm{C} 001 \mathrm{~B}$ & $\begin{array}{l}\text { HEPA FILTER 45C-FL- } \\
\text { 2B PRE-FILTER PDI }\end{array}$ & OPERATING: 1.2 IN & (DOC. C-13K1-001, V-46) \\
\hline PDI - 45C001C & $\begin{array}{l}\text { HEPA FILTER 45C-FL- } \\
\text { 2C PRE-FILTER PDI } \\
\end{array}$ & OPERATING: 1.2 IN & (DOC. C-13K1-001, V-46) \\
\hline PDI $-45 \mathrm{C} 002 \mathrm{~A}$ & $\begin{array}{l}\text { HEPA FILTER } 45 \mathrm{C} \cdot \mathrm{FL}- \\
\text { 2A HEPA PDI }\end{array}$ & OPERATING: $4.0 \mathrm{IN}$ & (DOC. C-13K1-001, V-46) \\
\hline PDI $-45 \mathrm{COO2B}$ & $\begin{array}{l}\text { HEPA FILTER } 45 \mathrm{C}-\mathrm{FL}- \\
\text { 2B HEPA PDI }\end{array}$ & OPERATING: $4.0 \mathrm{IN}$ & (DOC. C-13K1-001, V-46) \\
\hline PDI $-45 \mathrm{C} 002 \mathrm{C}$ & $\begin{array}{l}\text { HEPA FILTER } 45 \mathrm{C}-\mathrm{FL}- \\
2 \mathrm{C} \text { HEPA PDI }\end{array}$ & OPERATING: 4.0 IN & (DOC. C-13K1-001, V-46) \\
\hline PI - 45A008 & $\begin{array}{l}\text { HEPA FILTER INLET } \\
\text { HEADER AIR PRESSURE }\end{array}$ & OPERATING: $-4.5 \mathrm{IN}$ & (DOC. C-13K1-001, V-46) \\
\hline$T E-45 B 001 A$ & $\begin{array}{l}\text { PRE HEAT COIL OUTLET } \\
\text { AIR TEMP. SENSOR }\end{array}$ & TS : $40^{\circ} \mathrm{F}$ & $\begin{array}{l}\text { ENERGIZE ELECTRIC PREHEAT COIL } \\
\text { 2025E-45B-E-1A (BY LOCAL CONTROLLER) }\end{array}$ \\
\hline
\end{tabular}


TABLE $30 \quad 45$ HVAC - RCA

\begin{tabular}{|c|c|c|c|}
\hline $\begin{array}{l}\text { Tag No. PID No. } \\
\text { H-2-89021(1/2) }\end{array}$ & Name & Set-Values and Functions & Selection Basis \\
\hline$T E-45 B 001 B$ & $\begin{array}{l}\text { PRE HEAT COIL OUTLET } \\
\text { AIR TEMP. SENSOR } \\
\end{array}$ & TS : $40^{\circ} \mathrm{F}$ & $\begin{array}{l}\text { ENERGIZE ELECTRIC PREHEAT COIL 2025E-45B- } \\
\text { E-1B }\end{array}$ \\
\hline TE-45B005 & $\begin{array}{l}\text { SUPPLY AIR DUCT } \\
\text { TEMP. }\end{array}$ & OPERATING: $62^{\circ} \mathrm{F}$ & System Design \\
\hline $\mathrm{TI}-45 \mathrm{A002}$ & $\begin{array}{l}\text { HEPA FILTER INLET } \\
\text { HEADER AIR TEMP. }\end{array}$ & OPERATING: $65^{\sim} 82^{\circ} \mathrm{F}$ & System Design \\
\hline TIC - 45A001-1 & ROOM TEMP. SENSORS & $\begin{array}{l}\text { SV: } \text { OPERATOR SELECTED } \\
\text { AH: } 86^{\circ} \mathrm{F} \\
\text { AL: } 61^{\circ} \mathrm{F} \\
\text { TSH: } 85^{\circ} \mathrm{F} \\
\text { TSL: } 83^{\circ} \mathrm{F} \\
\end{array}$ & $\begin{array}{l}\text { CONTROL CHILLED WATER FLOW, CONTROL } \\
\text { EVAPORATIVE MEDIA UNIT PUMP 2025E-RTB-EM- } \\
\text { 1A/1B }\end{array}$ \\
\hline TIC-45A001-2 & ROOM TEMP. SENSORS & Sv: $65^{\bullet} \mathrm{F}$ & $\begin{array}{l}\text { CONTROL FINAL HEATING COIL 2025E-45B-E- } \\
2 \mathrm{~A} / 2 \mathrm{~B}\end{array}$ \\
\hline TIC-45A006 & ROOM 132 SPACE TEMP. & $\begin{array}{l}\text { SV: } \text { OPERATOR SELECTED } \\
\text { AH: } 105^{\circ} \mathrm{F} \\
\text { AL: } 61^{\circ} \mathrm{F}\end{array}$ & $\begin{array}{l}\text { ENERGIZED FAN ( } 45 B-E V U-F-5) \& \\
\text { MODULATE TY-45A006 }\end{array}$ \\
\hline TIC-45A007 & ROOM 139 SPACE TEMP. & $\begin{array}{l}\text { SV: } \text { OPERATOR SELECTED } \\
\text { AH: } 105^{\circ} \mathrm{F} \\
\text { AL: } 61^{\circ} \mathrm{F}\end{array}$ & $\begin{array}{l}\text { ENERGIZED FAN (45B-EVU-F-6) } \& \\
\text { MODULATE TY }-45 \text { A007 }\end{array}$ \\
\hline RI - 45E001A & & $\mathrm{AH}:$ & TO BE DETERMINED BY OPERATOR \\
\hline RI -45E001B & & $\mathrm{AH}:$ & TO BE DETERMINED BY OPERATOR \\
\hline $\mathrm{RI}-45 \mathrm{E} 001 \mathrm{C}$ & & AH: & TO BE DETERMINED BY OPERATOR \\
\hline RI - 45E001D & & AH: & TO BE DETERMINED BY OPERATOR \\
\hline
\end{tabular}


TABLE $30 \quad 45$ HVAC - NON RCA

\begin{tabular}{|c|c|c|c|}
\hline $\begin{array}{l}\text { Tag No. PID No. } \\
\mathrm{H}-2-89021(2 / 2)\end{array}$ & Name & Set-Values and Functions & Selection Basis \\
\hline II - 45B002 & $\begin{array}{l}\text { AIR HANDLING UNIT } \\
45 \mathrm{~B}-\mathrm{EVU}-6\end{array}$ & OPERATING: $16 \mathrm{~A}$ & FULL LOAD AMPERE: $19.6 \mathrm{~A}$ \\
\hline II - 45B003 & $\begin{array}{l}\text { AIR HANDLING UNIT } \\
45 \mathrm{~B} \text {-EVU- } 7\end{array}$ & OPERATING: $2.5 \mathrm{~A}$ & FULL LOAD AMPERE: $3.1 \mathrm{~A}$ \\
\hline II $-45 B 004$ & $\begin{array}{l}\text { AIR HANDLING UNIT } \\
45 \mathrm{~B}-\mathrm{EVU}-8\end{array}$ & OPERATING : $11 \mathrm{~A}$ & FULL LOAD AMPERE: $14 \mathrm{~A}$ \\
\hline PDI $-45 B 004$ & $\begin{array}{l}\text { AIR HANDLING UNIT } \\
45 \mathrm{~B}-\mathrm{FL}-2 \text { FILTER PDI }\end{array}$ & OPERATING: 3.8 IN & (DOC. C-13K1-001, VI-49) \\
\hline PDI $-45 B 005$ & $\begin{array}{l}\text { AIR HANDLING UNIT } \\
45 \mathrm{~B}-\mathrm{FL}-3 \text { FILTER PDI }\end{array}$ & OPERATING: $3.8 \mathrm{IN}$ & (DOC. C-13K1-001, VI-49) \\
\hline PDI $-45 B 006$ & $\begin{array}{l}\text { AIR HANDLING UNIT } \\
\text { 45B-FL-4 FILTER PDI }\end{array}$ & OPERATING: $1.9 \mathrm{IN}$ & (DOC. C-13K1-001, VI-49) \\
\hline PDI - 45B007 & $\begin{array}{l}\text { AIR HANDLING UNIT } \\
\text { 45B-FL-5 FILTER PDI }\end{array}$ & OPERATING: 1.9 IN & (DOC. C-13K1-001, VI-49) \\
\hline TI $-45 A 003$ & ROOM 107 TEMP. & $\begin{array}{l}\text { OPERATING: } 72^{\circ} \mathrm{F} \pm 2^{\circ} \mathrm{F} \\
\text { AL } 68^{\circ} \mathrm{F} \\
\text { AH } 82^{\circ} \mathrm{F}\end{array}$ & System Design \\
\hline TI $-45 A 004$ & ROOM 126 TEMP. & $\begin{array}{l}\text { OPERATING: } 72^{\circ} \mathrm{F} \pm 2^{\circ} \mathrm{F} \\
\text { AL } 68^{\circ} \mathrm{F} \\
\text { AH } 82^{\circ} \mathrm{F}\end{array}$ & System Design \\
\hline TI $-45 A 005$ & CONTROL ROOM TEMP. & $\begin{array}{l}\text { OPERATING: } 72^{\circ} \mathrm{F} \pm 2^{\circ} \mathrm{F} \\
\text { AL } 68^{\circ} \mathrm{F} \\
\text { AH } 76^{\circ} \mathrm{F}\end{array}$ & CONTROL POINT: $72^{\circ} \mathrm{F}$ \\
\hline$T I-45 B 006$ & $\begin{array}{l}\text { AIR HANDLER UNIT } \\
45 B-\text { EVU-6 SUPPLY AIR } \\
\text { TEMP. }\end{array}$ & OPERATING: $57.5^{\circ} \mathrm{F}$ & (DOC C-13K1-001, VII-33) \\
\hline TI - 45B007 & $\begin{array}{l}\text { AIR HANDLER UNIT } \\
45 \mathrm{~B}-\text { EVU-7 SUPPLY AIR } \\
\text { TEMP. }\end{array}$ & OPERATING: $66^{\circ} \mathrm{F}$ & (DOC C-13K1-001, VIII-18) \\
\hline TI $-45 B 008$ & $\begin{array}{l}\text { AIR HANDLER UNIT } \\
45 B-\text { EVU-8 SUPPLY AIR } \\
\text { TEMP. }\end{array}$ & OPERATING: $57.5^{\circ} \mathrm{F}$ & (DOC C-13K1-001, VII-33) \\
\hline
\end{tabular}


TABLE $30 \quad 45$ HVAC - NON RCA

\begin{tabular}{|c|c|c|c|}
\hline $\begin{array}{l}\text { Tag No PID No, } \\
\mathrm{H}-2-89021(2 / 2)\end{array}$ & Name & Set-Values and Functions & Selection Basis \\
\hline 45B-EVU- 6 & ROOM TEMP. CONT. & $\begin{array}{l}\text { SV: } \\
78^{\circ} F(S), 72^{\circ} F(W)\end{array}$ & $\begin{array}{l}\text { ENERGIZED FAN (45B-EVU-F-2) \& CONDENSING } \\
\text { UNIT (EVU-3) INSIDE OF AIR HANDLING UNIT } \\
(2025 \text { E-RTB-EVU-6) }\end{array}$ \\
\hline 45B-EVU-7 & ROOM TEMP. CONT. & $\begin{array}{l}\text { SV: } \\
78^{\circ} \mathrm{F}(\mathrm{S}), 72^{\circ} \mathrm{F}(\mathrm{W})\end{array}$ & $\begin{array}{l}\text { ENERGIZED FAN ( } 45 B-E V U-F-3) \text { \& CONDENSING } \\
\text { UNIT (EVU-4) INSIDE OF AIR HANDLING UNIT } \\
(2025 \text { E-RTB-EVU-7) }\end{array}$ \\
\hline
\end{tabular}


TABLE 31 45B HVAC CHILLED WATER (Chiller System)

\begin{tabular}{|c|c|c|c|}
\hline $\begin{array}{l}\text { Tag No. PID No. } \\
\text { H-2-89325 }\end{array}$ & Name & Set-Values and Functions & Selection Basis \\
\hline FI $-45 B 001 A$ & $\begin{array}{l}\text { AIR COOLED WATER } \\
\text { CHILLER INLET FLOW } \\
\text { (A) }\end{array}$ & OPERATING: $286 \mathrm{GPM}$ & System Design \\
\hline $\mathrm{FI}-45 \mathrm{~B} 001 \mathrm{~B}$ & $\begin{array}{l}\text { AIR COOLED WATER } \\
\text { CHILLER INLET FLOW } \\
\text { (B) }\end{array}$ & OPERATING: $286 \mathrm{GPM}$ & System Design \\
\hline$F I-45 B 002 A$ & $\begin{array}{l}\text { AIR HANDL_ING UNIT } \\
45 B-E V U-1 A \text { INLET } \\
\text { FLOW }\end{array}$ & OPERATING: $490 \mathrm{GPM}$ & System Design \\
\hline FI $-45 B 002 B$ & $\begin{array}{l}\text { AIR HANDLING UNIT } \\
45 \mathrm{~B}-\text { EVU-1B INLET } \\
\text { FLOW }\end{array}$ & OPERATING: $490 \mathrm{GPM}$ & System Design \\
\hline FI-45B003 & $\begin{array}{l}\text { AIR HANDLING UNIT } \\
45 \mathrm{~B}-\text { EVU-9 INLET FLOW }\end{array}$ & OPERATING: $28 \mathrm{GPM}$ & System Design \\
\hline F1-45B004 & $\begin{array}{l}\text { AIR HANDLING UNIT } \\
45 \mathrm{~B}-\text { EVU- } 10 \text { INLET } \\
\text { FLOW }\end{array}$ & OPERATING: $30 \mathrm{GPM}$ & System Design \\
\hline FS $-45 B 005 C$ & MINIMUM FLOW SW & SV: 120 GPM & System Design \\
\hline FS -45B005D & MINIMUM FLOW SW & SV: $120 \mathrm{GPM}$ & System Design \\
\hline II - 45B005A & $\begin{array}{l}\text { CHILLED WATER } \\
\text { CIRCULATION PUMP } \\
45 B-P-1 A \\
\text { CURRENT } \\
\end{array}$ & OPERATING: $40 \mathrm{~A}$ & FULL LOAD AMP. 62A (DOC. V-13K1-003-701) \\
\hline II $-45 B 005 B$ & $\begin{array}{l}\text { CHILLED WATER } \\
\text { CIRCULATION PUMP } \\
45 \mathrm{~B}-\mathrm{P}-1 \mathrm{~B} \\
\text { CURRENT }\end{array}$ & OPERATING: $40 \mathrm{~A}$ & FULL LOAD AMP. 62A (DOC. V-13K1-003-701) \\
\hline MAHL-45B001 & $\begin{array}{l}\text { CONTROL ROOM } \\
\text { HUMIDITY } \\
\end{array}$ & $\begin{array}{l}\text { AL } 30 \% \\
\text { AH } 80 x \\
\end{array}$ & System Design \\
\hline MI -45B001 & $\begin{array}{l}\text { CONTROL ROOM } \\
\text { HUMIDITY }\end{array}$ & OPERATING: $45 \%$ & System Design \\
\hline
\end{tabular}


TABLE 31 45B HVAC CHILLED WATER (Chlller System)

\begin{tabular}{|c|c|c|c|}
\hline $\begin{array}{l}\text { Tag No. PID No. } \\
\text { H-2-89325 }\end{array}$ & Name & Set-Values and Functions & Selection Basis \\
\hline MIC-45B002 & $\begin{array}{l}\text { HUMIDIFIER } \\
\text { 45B-MV-1 HUMIDITY }\end{array}$ & SV: $45 x$ & System Design \\
\hline $\mathrm{PI}-45 \mathrm{~B} 001$ & $\begin{array}{l}\text { MAKE-UP LINE } \\
\text { PRESSURE } \\
\end{array}$ & OPERATING: < 2OPSIG & PCV45B079; SET 20 PSIG \\
\hline PI-45B005 & $\begin{array}{l}\text { CHILLED WATER } \\
\text { CIRCULATION PUMP } \\
\text { OUTLET PRESSURE } \\
\end{array}$ & OPERATING: 93 PSIG & (DOC. V-13K1-003-701) \\
\hline PI -45B006A & $\begin{array}{l}\text { AIR HANDLING UNIT } \\
\text { 45B-EVU-1A INLET } \\
\text { PRESSURE }\end{array}$ & OPERATING: 90 PSIG & System Design \\
\hline PI - 45B006B & $\begin{array}{l}\text { AIR HANDLING UNIT } \\
45 B-E V I-1 B \text { INLET } \\
\text { PRESSURE }\end{array}$ & OPERATING: 90 PSIG & System Design \\
\hline PI - 45B008 & $\begin{array}{l}\text { AIR HANDLING UNIT } \\
45 B \text {-EVU-9 INLET } \\
\text { PRESSURE }\end{array}$ & OPERATING: 90 PSIG & System Design \\
\hline PI - 45B010 & $\begin{array}{l}\text { AIR HANDLING UNIT } \\
45 \mathrm{~B}-\text { EVU-10 INLET } \\
\text { PRESSURE }\end{array}$ & OPERATING: 90 PSIG & System Design \\
\hline PI - 45B011A & $\begin{array}{l}\text { AIR HANDLING UNIT } \\
45 B \text {-EVU-1A OUTLET } \\
\text { PRESSURE }\end{array}$ & OPERATING: $0-90$ PSIG & System Design \\
\hline PI - 45B011B & $\begin{array}{l}\text { AIR HANDLING UNIT } \\
45 B-\text { EVU-1B OUTLET } \\
\text { PRESSURE }\end{array}$ & OPERATING: $0-90$ PSIG & System Design \\
\hline PI-45B012 & $\begin{array}{l}\text { AIR HANDLING UNIT } \\
45 B-E V U-9 \text { OUTLET } \\
\text { PRESSURE }\end{array}$ & OPERATING: $0-90$ PSIG & System Design \\
\hline PI - 45B013 & $\begin{array}{l}\text { AIR HANDLING UNIT } \\
45 B-E V U-10 \text { OUTLET } \\
\text { PRESSURE }\end{array}$ & OPERATING: $0-90$ PSIG & System Design \\
\hline PI -45B014 & $\begin{array}{l}\text { Circulation Pump } \\
\text { Suction }\end{array}$ & OPERATING: - 30 PSIG & System Design \\
\hline
\end{tabular}


TABLE 31 45B HVAC CHILLED WATER (Chiller System)

\begin{tabular}{|c|c|c|c|}
\hline $\begin{array}{l}\text { Tag No. PID No. } \\
\text { H-2-89325 }\end{array}$ & Name & Set-Values and Functions & Selection Basis \\
\hline TI $-45 B 009$ & $\begin{array}{l}\text { AIR COOLED WATER } \\
\text { CHILLER INLET TEMP. }\end{array}$ & OPERATING: $45^{\circ}-55^{\circ} \mathrm{F}$ & System Design \\
\hline $\mathrm{TI}-45 \mathrm{~B} 010$ & $\begin{array}{l}\text { CHILLED WATER } \\
\text { CIRCULATION PUMP } \\
\text { INLET TEMP. }\end{array}$ & OPERATING: $45^{\circ} \sim 55^{\circ} \mathrm{F}$ & System Design \\
\hline $45 B-E V U-2 A$ & $\begin{array}{l}\text { AIR COOLED WATER } \\
\text { CHILLER } 45 \mathrm{~B}-\text { EVU-2A }\end{array}$ & $\begin{array}{l}\text { SV: } 44^{\circ} \mathrm{F} \\
\text { (LWT } 44^{\circ} \mathrm{F} \\
\end{array}$ & System Design \\
\hline 45B-EVU-2B & $\begin{array}{l}\text { AIR COOLED WATER } \\
\text { CHILLER } 45 A-E V U-2 B\end{array}$ & $\begin{array}{l}\text { SV: } 44^{\circ} \mathrm{F} \\
\left(\text { LWT } 44^{\circ} \mathrm{F} \quad \text { EWT } 55^{\circ} \mathrm{F} \text { ) }\right.\end{array}$ & System Design \\
\hline
\end{tabular}

密 
TABLE 3268 - 200 AREA TREATED EFFLUENT DISPOSAL FACILITY

\begin{tabular}{|c|c|c|c|c|}
\hline Tag No. & Name & Set-V & lues and Functions & Selection Basis \\
\hline TI 68A056 & $\begin{array}{l}\text { BLDG. } 225 \text {-W (PUMP } \\
\text { STATION } 1 \text { BLDG.) AIR } \\
\text { TEMP. }\end{array}$ & $\begin{array}{l}\text { AAHH: } \\
\text { AAH: } \\
\text { AAL: } \\
\text { AALL: }\end{array}$ & $\begin{array}{r}105^{\circ} \mathrm{F} \\
85^{\circ} \mathrm{F} \\
50^{\circ} \mathrm{F} \\
40^{\circ} \mathrm{F} \\
\end{array}$ & $\begin{array}{l}\text { DESIGN CALCULATION \#W049H-053 } \\
\text { VI } 22600 \text {, SUPP } 33\end{array}$ \\
\hline TI $68 \mathrm{~B} 046$ & $\begin{array}{l}\text { BLDG. } 225-\text { E (PUMP } \\
\text { STATION } 2 \text { BLDG.) AIR } \\
\text { TEMP. }\end{array}$ & $\begin{array}{l}\text { AAHH : } \\
\text { AAH: } \\
\text { AAL: } \\
\text { AALL: }\end{array}$ & $\begin{array}{r}105^{\circ} \mathrm{F} \\
85^{\circ} \mathrm{F} \\
50^{\circ} \mathrm{F} \\
40^{\circ} \mathrm{F} \\
\end{array}$ & $\begin{array}{l}\text { DESIGN CALCULATION \#W049H-053 } \\
\text { VI } 22600, \text { SUPP } 33\end{array}$ \\
\hline TI $68 \mathrm{C} 009$ & $\begin{array}{l}\text { BLDG. } 6653 \text { (DISPOSAL } \\
\text { BLDG.) AIR TEMP. }\end{array}$ & $\begin{array}{l}\text { AAHH: } \\
\text { AAH: } \\
\text { AAL: } \\
\text { AALL: }\end{array}$ & $\begin{array}{r}105^{\circ} \mathrm{F} \\
85^{\circ} \mathrm{F} \\
50^{\circ} \mathrm{F} \\
40^{\circ} \mathrm{F} \\
\end{array}$ & $\begin{array}{l}\text { DESIGN CALCULATION \#W049H-053 } \\
\text { VI } 22600 \text {, SUPP } 33\end{array}$ \\
\hline TI 68WA001 & $\begin{array}{l}\text { BLDG. } 225 \text {-WA (T } \\
\text { PLANT LCU BLDG.) AIR } \\
\text { TEMP }\end{array}$ & $\begin{array}{l}\text { AAHH: } \\
\text { AAH: } \\
\text { AAL: } \\
\text { AALL: }\end{array}$ & $\begin{array}{r}105^{\circ} \mathrm{F} \\
85^{\circ} \mathrm{F} \\
50^{\circ} \mathrm{F} \\
40^{\circ} \mathrm{F} \\
\end{array}$ & $\begin{array}{l}\text { DESIGN CALCULATION \#W049H-053 } \\
\text { VI } 22600 \text {, SUPP } 33\end{array}$ \\
\hline TI $68 \mathrm{WB} 001$ & $\begin{array}{l}\text { BLDG. } 225-\text { WB }(222-S \\
\text { LCU BLDG.) AIR TEMP. }\end{array}$ & $\begin{array}{l}\text { AAHH: } \\
\text { AAH: } \\
\text { AAL: } \\
\text { AALL: }\end{array}$ & $\begin{array}{r}105^{\circ} \mathrm{F} \\
85^{\circ} \mathrm{F} \\
50^{\circ} \mathrm{F} \\
40^{\circ} \mathrm{F} \\
\end{array}$ & $\begin{array}{l}\text { DESIGN CALCULATION \#W049H-053 } \\
\text { VI } 22600 \text {, SUPP } 33\end{array}$ \\
\hline TI 68WC001 & $\begin{array}{l}\text { BLDG. 225-WC (PFP } \\
\text { LCU BLDG.) AIR TEMP }\end{array}$ & $\begin{array}{l}\text { AAHH: } \\
\text { AAH: } \\
\text { AAL: } \\
\text { AALL: }\end{array}$ & $\begin{array}{r}105^{\circ} \mathrm{F} \\
85^{\circ} \mathrm{F} \\
50^{\circ} \mathrm{F} \\
40^{\circ} \mathrm{F} \\
\end{array}$ & $\begin{array}{l}\text { DESIGN CALCULATION \#WO49H-053 } \\
\text { VI } 22600 \text {, SUPP } 33\end{array}$ \\
\hline TI 68WE001 & $\begin{array}{l}\text { BLDG. } 283-W C(284-W \\
\text { LCU BLDG.) AIR TEMP }\end{array}$ & $\begin{array}{l}\text { AAHH: } \\
\text { AAH: } \\
\text { AAL: } \\
\text { AALL: }\end{array}$ & $\begin{array}{r}105^{\circ} \mathrm{F} \\
85^{\circ} \mathrm{F} \\
50^{\circ} \mathrm{F} \\
40^{\circ} \mathrm{F} \\
\end{array}$ & $\begin{array}{l}\text { DESIGN CALCULATION \#W049H-053 } \\
\text { VI } 22600, \text { SUPP } 33\end{array}$ \\
\hline TI 68EC001 & $\begin{array}{l}\text { 225-EC (PUREX LCU } \\
\text { BLDG.) AIR TEMP. }\end{array}$ & $\begin{array}{l}\text { AAHH: } \\
\text { AAH: } \\
\text { AAL: } \\
\text { AALL: }\end{array}$ & $\begin{array}{r}105^{\circ} \mathrm{F} \\
85^{\circ} \mathrm{F} \\
50^{\circ} \mathrm{F} \\
40^{\circ} \mathrm{F} \\
\end{array}$ & $\begin{array}{l}\text { DESIGN CALCULATION \#WO49H-053 } \\
\text { VI } 22600 \text {, SUPP } 33\end{array}$ \\
\hline IAH $68 A 012$ & PUMP 68A-P-A1 & FLA: & 36.5 & $\begin{array}{l}\text { DESIGN SERVICE FACTOR } \\
\text { FLA: Full Load Amperage }\end{array}$ \\
\hline
\end{tabular}


TABLE 3268 - 200 AREA TREATED EFFLUENT DISPOSAL FACILITY

\begin{tabular}{|c|c|c|c|c|}
\hline Tag No. & Name & \multicolumn{2}{|c|}{ Set-Values and Functions } & Selection Basis \\
\hline IAH $68 \mathrm{A026}$ & PUMP 68A-P-A2 & FLA : & 81.0 & $\begin{array}{l}\text { DESIGN SERVICE FACTOR } \\
\text { FLA: Full Load Amperage }\end{array}$ \\
\hline IAH $68 \mathrm{A0} 38$ & PUMP 68A-P-B1 & FLA: & 36.5 & $\begin{array}{l}\text { DESIGN SERVICE FACTOR } \\
\text { FLA: Full Load Amperage }\end{array}$ \\
\hline IAH $68 A 046$ & PUMP 68A-P-B2 & FLA : & 81.0 & $\begin{array}{l}\text { DESIGN SERVICE FACTOR } \\
\text { FLA: Full Load Amperage }\end{array}$ \\
\hline IAH $68 B 012$ & PUMP 68B-P-A1 & FLA: & 36.5 & $\begin{array}{l}\text { DESIGN SERVICE FACTOR } \\
\text { FLA: Full Load Amperage }\end{array}$ \\
\hline IAH $68 B 028$ & PUMP 68B-P-B1 & FLA: & 36.5 & $\begin{array}{l}\text { DESIGN SERVICE FACTOR } \\
\text { FLA: Full Load Amperage }\end{array}$ \\
\hline LE-68A004 & $\begin{array}{l}\text { BLDG. } 225-W, \text { PUMP } \\
\text { STATION } 1 \text { WETWELL } \\
\text { SIDE A LEVEL }\end{array}$ & $\begin{array}{l}\text { LAH: } \\
\text { LSHH: } \\
\text { LSH: } \\
\text { LSL: }\end{array}$ & $\begin{array}{ll}72 & \text { INCHES } \\
60 & \text { INCHES } \\
54 & \text { INCHES } \\
36 & \text { INCHES } \\
\end{array}$ & SYSTEM DESIGN \\
\hline$L E-68 A 030$ & $\begin{array}{l}\text { BLDG, } 225-W, \text { PUMP } \\
\text { STATION } 1 \text { WETWELL } \\
\text { SIDE B LEVEL }\end{array}$ & $\begin{array}{l}\text { LAH: } \\
\text { LSHH: } \\
\text { LSH: } \\
\text { LSL: }\end{array}$ & $\begin{array}{ll}72 & \text { INCHES } \\
60 & \text { INCHES } \\
54 & \text { INCHES } \\
36 & \text { INCHES } \\
\end{array}$ & SYSTEM DESIGN \\
\hline$L E-68 A 005$ & $\begin{array}{l}\text { BLDG. } 225-W \text {, PUMP } \\
\text { STATION } 1 \text { WETWELL } \\
\text { SIDE A LOW LEVEL } \\
\text { SWITCH }\end{array}$ & LAL: & 14 INCHES & SYSTEM DESIGN \\
\hline LE-68A031 & $\begin{array}{l}\text { BLDG. } 225-W \text {, PUMP } \\
\text { STATION } 1 \text { WETWELL } \\
\text { SIDE A LOW LEVEL } \\
\text { SWITCH }\end{array}$ & LAL: & 12 INCHES & SYSTEM DESIGN \\
\hline LE- 68B004 & $\begin{array}{l}\text { BLDG. } 225 \text {-E, PUMP } \\
\text { STATION } 2 \text { WETWELL } \\
\text { SIDE A LEVEL }\end{array}$ & $\begin{array}{l}\text { LAH: } \\
\text { LSH: } \\
\text { LSL: }\end{array}$ & $\begin{array}{ll}54 & \text { INCHES } \\
48 & \text { INCHES } \\
36 & \text { INCHES } \\
\end{array}$ & SYSTEM DESIGN \\
\hline LE-68B022 & $\begin{array}{l}\text { BLDG, } 225-E \text {, PUMP } \\
\text { STATION } 2 \text { WETWELL } \\
\text { SIDE B LEVEL }\end{array}$ & $\begin{array}{l}\text { LAH: } \\
\text { LSH: } \\
\text { LSL: }\end{array}$ & $\begin{array}{ll}54 & \text { INCHES } \\
48 & \text { INCHES } \\
36 & \text { INCHES } \\
\end{array}$ & SYSTEM DESIGN \\
\hline
\end{tabular}


TABLE 3268 - 200 AREA TREATED EFFLUENT DISPOSAL FACILITY

\begin{tabular}{|c|c|c|c|}
\hline Tag No. & Name & Set-Values and Functions & Selection Basis \\
\hline LE- 68B005 & $\begin{array}{l}\text { BLDG, } 225-E \text {, PUMP } \\
\text { STATION } 2 \text { WETWELL } \\
\text { SIDE A LOW LEVEL } \\
\text { SWITCH }\end{array}$ & LAL: $\quad 13$ INCHES & SYSTEM DESIGN \\
\hline LE - 68B023 & $\begin{array}{l}\text { BLDG. 225-E, PUMP } \\
\text { STATION } 2 \text { WETWELL } \\
\text { SIDE B LOW LEVEL } \\
\text { SWITCH }\end{array}$ & LAL: $\quad 10$ INCHES & SYSTEM DESIGN \\
\hline LE $-68 C 008$ & $\begin{array}{l}\text { DISPOSAL POND A } \\
\text { LEVEL }\end{array}$ & LAH: 108 INCHES & SYSTEM DESIGN \\
\hline LE - $68 \mathrm{C} 007$ & $\begin{array}{l}\text { DISPOSAL POND B } \\
\text { LEVEL }\end{array}$ & LAH: 144 INCHES & SYSTEM DESIGN \\
\hline AXS - $68 \mathrm{C} 012$ & DISPOSAL pH & $\begin{array}{ll}\text { AAH : } & 8.5 \\
\text { AAL: } & 6.5 \\
\end{array}$ & STATE WASTE DISCHARGE PERMIT \\
\hline AXS $68 \mathrm{C} 011$ & $\begin{array}{l}\text { DISPOSAL } \\
\text { CONDUCTIVITY }\end{array}$ & AAH: $750 \mathrm{uS}$ & OPERATING INDICATOR \\
\hline FI W8-001 & 222-S FLOW & FQI: 75 GPM & SYSTEM DESIGN \\
\hline FI $68 \mathrm{~A}-201$ & PFP FLOW & FQI : 500 GPM & SYSTEM DESIGN \\
\hline FI 284W004 & 284-W FLOW & FQI: $340 \mathrm{GPM}$ & SYSTEM DESIGN \\
\hline FI WA 100 & T PLANT FLOW & FQI : $110 \mathrm{GPM}$ & SYSTEM DESIGN \\
\hline FI U1511 & B PLANT FLOW & FQI: $200 \mathrm{GPM}$ & SYSTEM DESIGN \\
\hline FI RW-1/2 & 242-A-81 FLOW & FQI : $500 \mathrm{GPM}$ & SYSTEM DESIGN \\
\hline FI W20-1-1 & PUREX FLOW & FQI: $600 \mathrm{GPM}$ & SYSTEM DESIGN \\
\hline LI WA_100 & T PLANT LEVEL & $\begin{array}{l}\text { LAHH: } 90 x \\
\text { LALL: } 15 x \\
\end{array}$ & SYSTEM DESIGN \\
\hline RAH_WA_105 & $T$ PLANT BETA & $\begin{array}{l}\text { AAH: DIGITAL INPUT } \\
\text { SET BY T PIANT }\end{array}$ & SYSTEM DESIGN \\
\hline RAH NS2-1 & B PLANT BETA & AAH: $1000 \mathrm{CPM}$ & SYSTEM DESIGN \\
\hline RAH NS2-2 & B PLANT GAMMA & AAH: $\quad 1000 \mathrm{CPM}$ & SYSTEM DESIGN \\
\hline
\end{tabular}


TABLE 3268 - 200 AREA TREATED EFFLUENT DISPOSAL FACILITY

\begin{tabular}{|c|c|c|c|}
\hline Tag No. & Name & Set-Values and Functions & Selection Basis \\
\hline RAH W20-2-1 & PUREX BETA/GAMMA & AAH: $7000 \mathrm{CPM}$ & SYSTEM DESIGN \\
\hline TI 284W008 & 284-W EFFLUENT TEMP. & AAH: $100^{\circ} \mathrm{F}$ & SYSTEM DESIGN \\
\hline FXS - 68B100 & $\begin{array}{l}\text { H LINE FLOW METER } \\
\text { FAULT TIME DELAY }\end{array}$ & 10 SECONDS & SYSTEM DESIGN \\
\hline $68 \mathrm{~A}-\mathrm{P}-\mathrm{A} 1$ & PUMP SHUTOFF DELAY & 40 SECONDS & SYSTEM DESIGN \\
\hline 68A-P-A2 & PUMP SHUTOFF DELAY & 40 SECONDS & SYSTEM DESIGN \\
\hline $68 \mathrm{~A}-\mathrm{P}-\mathrm{B} 1$ & PUMP SHUTOFF DELAY & 40 SECONDS & SYSTEM DESIGN \\
\hline $68 \mathrm{~A}-\mathrm{P}-\mathrm{B} 2$ & PUMP SHUTOFF DELAY & 40 SECONDS & SYSTEM DESIGN \\
\hline $68 \mathrm{~B}-\mathrm{P}-\mathrm{A} 1$ & PUMP SHUTOFF DELAY & 40 SECONDS & SYSTEM DESIGN \\
\hline $68 \mathrm{~B}-\mathrm{P}-\mathrm{B} 1$ & PUMP SHUTOFF DELAY & 40 SECONDS & SYSTEM DESIGN \\
\hline $68 \mathrm{~A}-\mathrm{ECV}-03$ & $\begin{array}{l}\text { OPENING TIME } \\
\text { CLOSING TIME } \\
\end{array}$ & $\begin{array}{ll}20-35 & \text { SECONDS } \\
35-45 & \text { SECONDS } \\
\end{array}$ & SYSTEM DESIGN \\
\hline $68 A-E C V-20$ & $\begin{array}{l}\text { OPENING TIME } \\
\text { CLOS ING TIME } \\
\end{array}$ & $\begin{array}{ll}20-35 & \text { SECONDS } \\
35-45 & \text { SECONDS } \\
\end{array}$ & SYSTEM DESIGN \\
\hline $68 A-E C V-08$ & $\begin{array}{l}\text { OPENING TIME } \\
\text { CLOS ING TIME } \\
\end{array}$ & $\begin{array}{ll}20-35 & \text { SECONDS } \\
35-45 & \text { SECONDS } \\
\end{array}$ & SYSTEM DESIGN \\
\hline $68 A-E C V-25$ & $\begin{array}{l}\text { OPENING TIME } \\
\text { CLOSING TIME } \\
\end{array}$ & $\begin{array}{ll}20-35 & \text { SECONDS } \\
35-45 & \text { SECONDS } \\
\end{array}$ & SYSTEM DESIGN \\
\hline $68 \mathrm{~B}-\mathrm{ECV}-03$ & $\begin{array}{l}\text { OPENING TIME } \\
\text { CLOSING TIME } \\
\end{array}$ & $\begin{array}{ll}20-35 & \text { SECONDS } \\
35-45 & \text { SECONDS } \\
\end{array}$ & SYSTEM DESIGN \\
\hline $68 B-E C V-21$ & $\begin{array}{l}\text { OPENING TIME } \\
\text { CLOSING TIME }\end{array}$ & 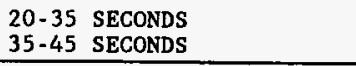 & SYSTEM DESIGN \\
\hline
\end{tabular}


APPENDIX A - PCV LIST - ALL SYSTEMS

\begin{tabular}{|c|c|c|c|c|c|c|c|c|c|}
\hline TAG & No & FLUID & OPE. & DESIGN RANGE & OPE.* & SET-POINT & FLOW RATE & P\&ID & REMARKS \\
\hline & & & (F) & (psig) & (psig) & (psig) & (GPM/SCFM) & & \\
\hline PCV1B & 037 & Air & 80 & $60-120$ & 110 & 100 & 390 & H2 - 89000 & ADTECHS \\
\hline PCV1B & 038 & Air & 80 & $60-120$ & 90 & 90 & 390 & $\mathrm{H} 2-89000$ & ADTECHS \\
\hline PCV1D & 031 & Air & 80 & $70-150$ & 90 & 90 & 100 & $\mathrm{H} 2-89336$ & ADTECHS \\
\hline PCV45B & 079 & Water & - & $10-35$ & 20 & 20 & & $\mathrm{H} 2-89325$ & TMC \\
\hline PSV60A & 106 & Air & 120 & $+2,-18{ }^{\prime \prime} \mathrm{H}^{2} \mathrm{O}$ & $-2^{n} \mathrm{H}^{2} \mathrm{O}$ & $+1,-94 \mathrm{H}^{2} \mathrm{O}$ & To Outdoor & $\mathrm{H} 2-89337$ & ADTECHS \\
\hline PCV60B & 023 & Air & 80 & 150 & 90 & 90 & - & H2-88975 & PALL \\
\hline PCV60B & 053 & A1r & 80 & 150 & 90 & 90 & - & $\mathrm{H} 2-88978$ & PALL \\
\hline PCV60F & 025 & Water & 80 & $65-130$ & 130 & 80 & 50 & H2-88979 & ADTECHS \\
\hline PCV60F & 042 & Water & 80 & $100-300$ & $300(500)$ & 130 & 50 & H2-88979 & ADTECHS \\
\hline PCV95D & 001 & water & 80 & $0-300$ & $160-170$ & 150 & - & H2-89023 & ADTECHS \\
\hline PGV60G & 032 & Air & 80 & 90 & 90 & 30 & 110 & H2-88983 & INDECK \\
\hline PCV60H & 092 & Water & 80 & $5-15 * *(150)$ & 72 & 13 & 0.8 & H2-88988 & ADTECHS \\
\hline PCV60H & 095 & Water & 80 & $25-75 * \star(120)$ & 90 & 25 & 150 & H2-89337 & ADTECHS \\
\hline PCV60I & 244 & Water & 122 & 150 & 50 & 50 & 20 & H2-89335 & RCC \\
\hline PCV60I & 245 & Water & 122 & 150 & 55 & 55 & 20 & H2-89335 & RCC \\
\hline PCV60I & 281 & Antifoam & 100 & 150 & 50 & 50 & $1 \star \star \star \star$ & $\mathrm{H}-2.89335$ & RCC \\
\hline PCV65C & 072 & $92 \pi$ & 80 & 150 & $<185$ & 75 & $10 * \star \star$ & H2 - 89337 & PULSA \\
\hline PCV65C & 073 & $50 x$ & 80 & 150 & $<100$ & 75 & $0.5 \star \star \star$ & H2-89337 & PULSA \\
\hline PCV65C & 095 & $92 x$ & 80 & 150 & $<150$ & 75 & 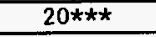 & $\mathrm{H} 2-89337$ & PULSA \\
\hline PCV65C & 097 & $4 \%$ & 80 & 150 & $<100$ & 75 & $10 \star \star \star$ & H2-88977 & PULSA \\
\hline PCV65C & 099 & $4 x$ & 80 & 150 & $<100$ & 75 & $10 * * *$ & $\mathrm{H} 2-88977$ & PULSA \\
\hline PCV65C & 118 & $4 \pi$ & 80 & 150 & $<100$ & 75 & $2 * * *$ & $\mathrm{H} 2-88977$ & PULSA \\
\hline PCV95D & 002 & Water & 80 & $25-75$ & $160-170$ & 50 & 100 & $\mathrm{H} 2-89023$ & ADTECHS \\
\hline PCV95D & 108 & Water & 80 & $25-75$ & 50 & 50 & 10 & $\mathrm{H} 2-89023$ & CULLIGAN \\
\hline $68 \mathrm{~A}-\mathrm{SAV}-12$ & & Water & Amb. & Adj: & 90 & $105 \pm 5$ & 1000 & $\begin{array}{c}\mathrm{H} 2-140378 \\
\mathrm{SH} 1\end{array}$ & GA INDUSTRIES \\
\hline
\end{tabular}


APPENDIX B - PSV LIST - ALL SYSTEMS

\begin{tabular}{|c|c|c|c|c|c|c|c|c|c|}
\hline TAG & No & FLUID & OPE. & DESIGN RANGE & OPE . * & SET - POINT & RELIEF LOCAT. & P\&ID & REMLARS \\
\hline & & & (F) & (psig) & $(p s i g)$ & (psig) & (GPM/SCFM) & & \\
\hline PSV1B & 036 & Air & 80 & 150 & 110 & 150 & To outdoor & H2-89000 & DICKENSON \\
\hline PSV1B & 037 & Air & 60 & 300 & 30 & 100 & To Room & H2-88983 & ADTECHS \\
\hline PSVID & 030 & Air & 80 & 150 & 90 & 175 & To outdoor & H2-89336 & DICKENSON \\
\hline PSV45D & 065 & Air & 80 & 1 & 0.3 & 1 & To room & $\mathrm{H} 2-88993$ & $\csc$ \\
\hline PSV60A & 096 & Water & 90 & 150 & 100 & 150 & To sump & H2-89337 & ADTECHS \\
\hline PSV60A & 106 & $\mathbf{A} \mathbf{R}$ & 120 & $-18^{\prime \prime} \mathrm{H} 2 \mathrm{O}$ & $-2^{2} \mathrm{H} 2 \mathrm{O}$ & $+1 /-9 " \mathrm{H} 2 \mathrm{O}$ & To Outdoor & H2-89337 & ADTECHS \\
\hline PSV60B & 011 & Water & $<140$ & 150 & $45-85$ & 150 & To sump & H2-88975 & PALL (185 gpm) \\
\hline PSV60B & 041 & Water & $<140$ & 150 & $45-85$ & 150 & To aump & H2-88978 & PALL (185 gpm) \\
\hline PSV60B & 061 & Air & 80 & 125 & 90 & 125 & To aump & H2-88975 & PALL \\
\hline PSV60B & 077 & Air & 80 & 125 & 90 & 125 & To room & H2-88978 & PALL \\
\hline PSV60D & 407 & Water & $<140$ & 150 & 85 & 150 & To sump & H2-89332 & PSI \\
\hline PSV60D & 419 & Water & $<140$ & 150 & 85 & 150 & To sump & H2-89332 & PSI \\
\hline PSV60F & 099 & Water & 70 & 600 & 10 & 600 & To Sump & H2-88980 & ADTECHS \\
\hline PSV60F & 170 & Water & 86 & 150 & 25 & 200 & To sump & H2-88980 & ZENON \\
\hline PSV60F & 247 & Water & 70 & 475 & 200 & 200 & To 60F-TK-1 & H2-88982 & ADTECKS \\
\hline PSV60F & 251 & Water & 60 & 600 & 10 & 600 & To Sump & H2-88981 & ADTECHS \\
\hline PSV60F & 285 & Water & 86 & 150 & 85 & 200 & To 60F-TK-1 & H2-88982 & 2ENON \\
\hline PSV60F & 288 & Water & 86 & 150 & 70 & 100 & To Sump & H2-88982 & ADTECHS \\
\hline PSV60I & 129 & Air & 248 & 150 & 15 & 25 & To sump & H2-889875 & RCC \\
\hline PSV601 & 131 & Water & 175 & 150 & 30 & 135 & To mump & H2-88987 & RCC \\
\hline PSV60I & 133 & Water & 213 & 150 & 84 & 135 & To sump & H2-88987 & RCC \\
\hline PSV60I & 168 & Steam & 228 & 150 & 15 & 25 & To outdoor & H2-89335 & RCC \\
\hline PSV60I & 203 & Steam & 249 & 150 & $15-50$ & 18.78 & To outdoor & H2-88987 & RCC \\
\hline PSV60I & 211 & Steam & 225 & 150 & 20 & 50 & To aump & H2-88987 & RCC \\
\hline PSV60I & 280 & Antifoem & 100 & 150 & 50 & 125 & To TK-04 & H2-89335 & $\overline{\mathrm{RCC}}$ \\
\hline PSV60I & 304 & Water & 94 & 150 & 100 & 110 & To wamp & H2-88987 & RCC \\
\hline PSV60J & 104 & Stenm & 365 & 300 & 150 & 165 & To mime & H2-88989 & LCI \\
\hline PSV60J & 211 & Water & 160 & 150 & 10 & 40 & To aump & H2-88989 & LCI \\
\hline PSV60J & 212 & Stenm & 200 & 150 & 15 & 150 & To minp & H2-88989 & LCI \\
\hline PSV65A & 023 & Air & - & 180 & 150 & 0 & From mom & H2-88999 & PRECISION \\
\hline PSV65A & 035 & Steum & 365 & 200 & 155 & 190 & To ang & H2-88999 & PRECISION \\
\hline PSV65A & 103 & Steam & 200 & 200 & 150 & 175 & To sump & H2-88999 & PRECISION \\
\hline PSV65A & 104 & Air & - & 300 & 150 & 0 & From room & H2-88999 & PRECISION \\
\hline PSV65A & 105 & Air & - & 300 & 5 & 0 & From noom & H2-88999 & PRECISION \\
\hline PSV65A & 106 & Steam & 228 & 180 & 5 & 15 & To sump & H2-88999 & PRECISION \\
\hline PSV95C & 121 & Water & 70 & 475 & 95 & 95 & To Sump & H2-88998 & DRESSER \\
\hline PSV95D & 003 & Water & 70 & 150 & 50 & 100 & To outdoor & H2-89023 & ADTECHS \\
\hline PSV95D & 109 & Water & 70 & 150 & 50 & 100 & To sump & H2-89023 & CULLIGAN \\
\hline
\end{tabular}




\begin{tabular}{|c|c|c|c|c|c|c|c|c|c|}
\hline TAG & NO & FLUID & OPE. & DESIGN RANGE & OPE ** & SET - POINT & RELIEF LOCAT. & P\&ID & REMARKS \\
\hline & & & (F) & $(p s i g)$ & $(p s i g)$ & (psig) & (GPM/SCFM) & & \\
\hline PSV95D & 115 & Water & 210 & 200 & 15 & 150 & To Pipe PIT & H2-88999 & ADTECHS \\
\hline PSV60J & 213 & Stenm & 212 & 150 & 0 & 15 & To sump & H2-88989 & LCI (RUPTURE DISC) \\
\hline PSV-58C-001 & & Water & Amb. & & & 25 & To aump & F12-815120SH 1 & NUFRO \\
\hline PSV-68C-002 & & Water & Amb. & & & 25 & To anmp & H2-815120SH 1 & NUPRO \\
\hline PSV-68C-010 & & Water & Amb. & & & 50 & To sump & H2-815120 SH 1 & CROSBY \\
\hline
\end{tabular}


APPENDIX C - RO DATA SHEET - ALL SYSTEMS

\begin{tabular}{|c|c|c|c|c|c|c|c|c|c|c|c|c|c|}
\hline \multirow[t]{2}{*}{$T \omega m$ m. } & \multirow[t]{2}{*}{ paro wo; } & \multirow[t]{2}{*}{ neteriel } & \multirow[t]{2}{*}{ 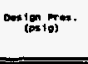 } & \multirow{2}{*}{ 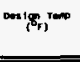 } & \multirow[t]{2}{*}{$\begin{array}{l}31(x) \\
(1 n)\end{array}$} & \multirow[t]{2}{*}{ Flowerete } & \multirow[t]{2}{*}{ Pren. (pist) } & \multirow[t]{2}{*}{ noof } & \multicolumn{2}{|c|}{$\$ 10$} & \multirow{2}{*}{ 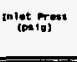 } & \multirow[t]{2}{*}{ semitino flow (s) } & \multirow[t]{2}{*}{ memerks } \\
\hline & & & & & & & & & (Inea) & $(-1$ & & & \\
\hline Fo-60x-oon & mis & 30058 & 100 & 200 & 3 & nin., & 3.56 & 1 & 1.301 & 34.ss & & 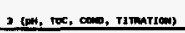 & \\
\hline ro-cen-esen & o933r & 3onss & 100 & 200 & 3 & $w_{2}$ & 10.21 & 1 & t.m & 3.10 & 54.10 & & \\
\hline ro-ten-ens & coms & sosss & 150 & 200 & 3 & 164.2 & 10.22 & $\perp$ & 1.461 & $3 r .20$ & 世.3. & $1.10 \pm$ & \\
\hline ro-con-ene & mers & .004ss & 150 & 300 & 3 & 169.2 & 21,33 & $\perp$ & 1.200 & 34,03 & 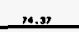 & 1.e $(\mathrm{mes})$ & \\
\hline 10-cecc.en1. & $\operatorname{con}$ & sonss & 150 & 200 & 3 & 160.7 & $2 . \infty$ & 1 & $2,0,2$ & S2.4. & 4.0 & $2,2,(0), \pi \infty)$ & $y=-$ vent \\
\hline 10.-cex-ese & 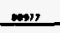 & monss & 150 & 2000 & $1-1 / 2$ & + & 21.4.4 & $\perp$ & 0.00 & 16.28 & n.2.4 & & \\
\hline Fo-60x-e0s & $m$ & senss & 190 & 200 & $1-1 / 2$ & 43.6 & 19.22 & 1 & 0.763 & 18,2 & no.e? & 1.8 (e) & \\
\hline c0-100.-000 & coms & 2004s & Lso & 100 & 3 & 100.0 & 14.22 & 1 & 1,46 & 37.25 & st.ut & 0.2 (nesen) & \\
\hline $80.400-0005$ & $\operatorname{mos}$ & $3045 s$ & 150 & 10 & ב & 10 & 14,22 & 1 & 1.44 & M.10 & 45,2 & 1 (Enier (CPS) & \\
\hline Fo-60F-001 & 6334 & 20458 & 150 & 120 & 3 & 16e.s & 14,22 & 1 & 1.450 & $3 r .001$ & 14,22 & 2.5 , & \\
\hline 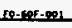 & men & man & $\infty$ & 130 & $1-14$ & 2 & 14.32 & 1 & 0.371 & 2.e. & 14.5s & $3(0 \mathrm{~d}, \mathrm{cos})$ & \\
\hline 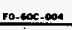 & enen & menss & 190 & 120 & 1-1/2 & 23 & 21,19 & 1 & t. soes & 12,2 & $n . m$ & & \\
\hline $10-40 x-005$ & m & sonss & 150 & 120 & $2-1 / 2$ & 21.5 & $.14,22$ & 1 & 0.540 & 12,11 & 50.20 & $1.5 \cos 2$ & \\
\hline 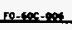 & $\mathrm{mm}$ & mass & Ne & 120 & 3. & 14.5 & $31, n$ & 1 & with & 2A:23 & athen & 2.5 $(\mathrm{csen}, \mathrm{AR})$ & \\
\hline F0.-\$11..001 & 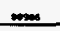 & sonss & 150 & 200 & 3 & 120 & 10.22 & 1 & 1.021 & $\mathbf{x . 2 4}$ & 44,65 & & \\
\hline Fo-_el-eove & 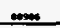 & 3004ss & 150 & 200 & 3 & 157 & 19.20 & 1 & 1.415 & s.es & 30.43 & $3(104, \infty, \infty)$ & \\
\hline 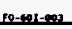 & m & gass & $1 \% 0$ & 200 & 3 & 14: & 14.39 & $ـ$ & $1.62 y$ & .t.24 & 4.65 & & \\
\hline Fo. & $m$ & sonss & 150 & 200 & 3 & 15r & 14.20 & 1 & 1.415 & $\mathrm{ms.m}$ & 30.43 & $3(\mathrm{pu}, \mathrm{cos})$ & \\
\hline P. & $=$ & Ilewss & 35 & 230 & $-3=1 / 2$ & 30 & 12.62 & 1 & 0.628 & 1...m & $\omega .$. & & \\
\hline 烈 & . & 314ess & ts & 230 & $1-1 / 2$ & 33.5 & 14,32 & 2 & 0.465 & 12.w & 47.35 & $1.8(0.4)$ & 20111 \\
\hline r. & m & 31enss & 3 & 230 & $x-1 / z$ & 3 & $20 . .60$ & 1 & 0.026 & $11 . .0$ & . & & \\
\hline 舟 & 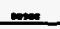 & sicess & 15 & 230 & $1-1 / 2$ & 32.5 & 14.22 & 2 & ستيك.0.0. & 11..8 & 0.35 & $1.8(0)$ & $20 x+1=1$ \\
\hline po-ren-eses & 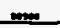 & Deass & $n$ & 3.00 & $3 / 4$ & 0.0 & w.en & 1 & 0.006 & 2.06 & n.n & & 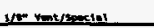 \\
\hline $10-200-\infty 10$ & $m$ & sonss & 100 & 120 & $1-1 / 2$ & 3.5 & 14.22 & 1 & 0.224 & s.en & 16.8 & & \\
\hline Fo-2000-1002 & -1 & mouss & 10 & 120 & 2 & 3.5 & 10.10 & 1 &. .246 & ...nt & 11.00 & $1.8(000)$ & \\
\hline Fo. $0.05 x_{-1002}$ & $m$ & enss & 150 & 120 & $1-3 / 2$ & 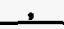 & $14 . n$ & 1 &. .412 & 10.4 & $14.2 n$ & & eass-nuess \\
\hline 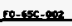 & $\operatorname{men}$ & 3asss & 35 & 100 & $3 / 4$ & سلر & $14, n$ & 1 & 0.310 & .02 & 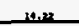 & & mans-nest:4 \\
\hline Fo-6sc.,e03 & m & 304ss & 1.50 & 120 & $1-1 / 2$ & 1.1 & 14.22 & 1 & 0.373 & 9.53 & 10.22 & & \\
\hline ED-arce-ton & $\max$ & 3onss & 25 & 120 & $3 / 4$ & 3.4 & 14.22 & & 0.316 & 0.02 & 16.22 & & \\
\hline E0-6x<-005 & Sex & mass & 15 & neo & $3 / 4$ & & & & & & & & Datrors \\
\hline ro- $-6 s c-.006$ & سيت & mass & ns & 120 & $3 / 4$ & & & & & & & & mente \\
\hline ro- $-5 x-600$ & 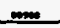 & monss & 75 & 120 & 34 & & & & & & & & Deter \\
\hline rouscamenen & $m$ & mass & LE & He & $3 / 4$ & & & & & & & & eacris. \\
\hline ro-sse-men & וيس & 314ss & $\infty$ & 10 & $1-\ln$ & & & 2 & $.393 \cdot .123$ & & & & 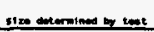 \\
\hline |0-9sc- & $\mathrm{mm}$ & es & $\omega$ & in & . & $\infty$ & sc.en & 1 & 2.316 & $\infty$ & 2.10 & & \\
\hline
\end{tabular}


WHC-SD-ETF-OCD-001, Rev. 2

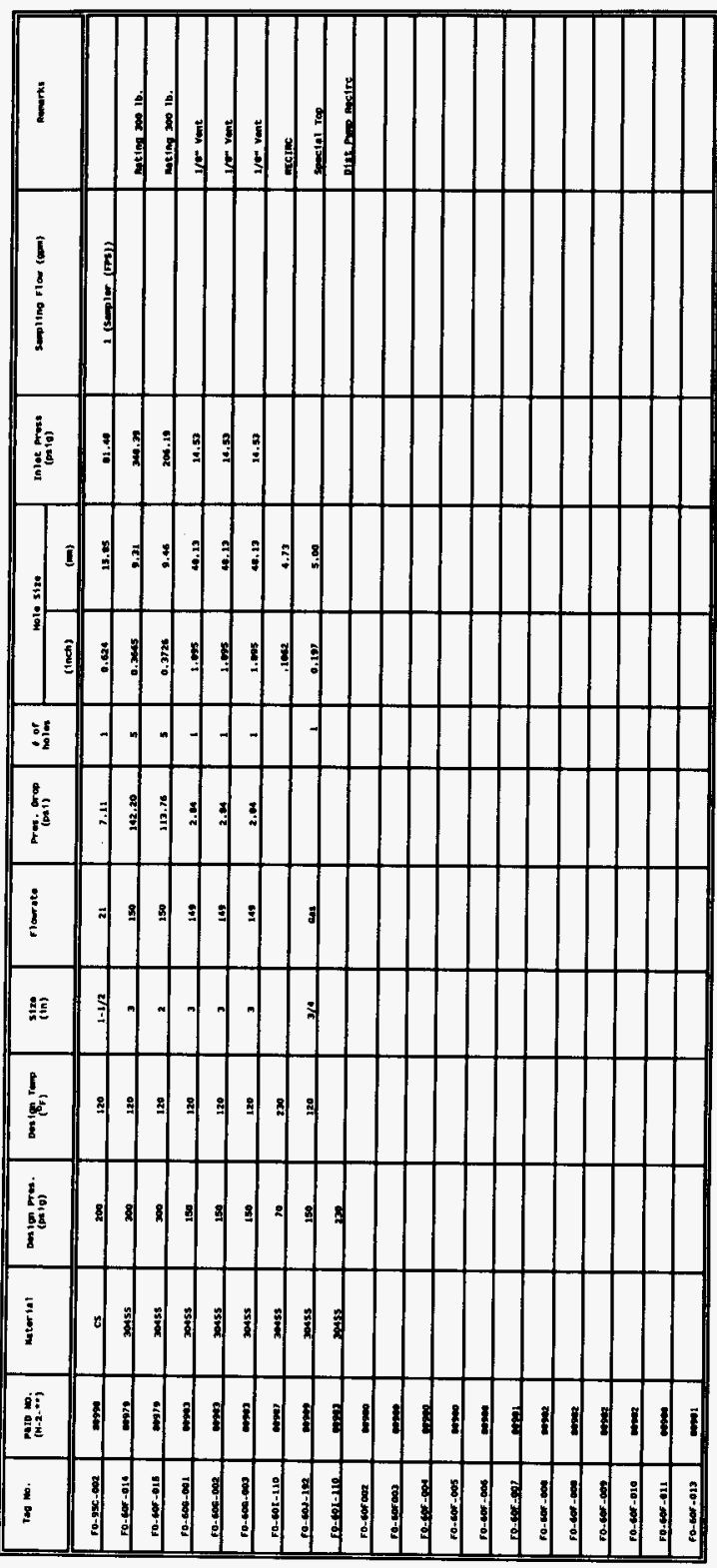

